\title{
Birationally rigid Pfaffian Fano 3-folds
}

\author{
Hamid Ahmadinezhad and Takuzo Okada
}

\begin{abstract}
We classify birationally rigid orbifold Fano 3 -folds of index 1 defined by $5 \times 5$ Pfaffian varieties. We give a sharp criterion for the birational rigidity of these families based on the type of singularities that the varieties admit. Various conjectures are born out of our study, highlighting a possible approach to the classification of terminal Fano 3 -folds. The birationally rigid cases are the first known rigid examples of Fano varieties that are not (weighted) complete intersections.
\end{abstract}

\section{Introduction}

A variety $X$ is Fano if its anticanonical class $-K_{X}$ is ample. Fano varieties are central in geometry, as any uniruled variety is birational to a Fano variety or a fibration into Fano varieties by the minimal model program (MMP).

Smooth Fano 3-folds have been classified by Iskovskikh [Isk77, Isk78] and Mori-Mukai [MM81]. However, when we look at Fano varieties as outputs of MMP, the smoothness condition must be relaxed and be replaced with $\mathbb{Q}$-factorial and terminal. The graded ring approach of Reid provides a list of Fano 3-folds to study. It considers a Fano 3-fold $X$ embedded into a weighted projective space via the anticanonical ring [ABR02]

$$
R\left(X,-K_{X}\right)=\bigoplus_{n \geqslant 0} \mathrm{H}^{0}\left(X,-n K_{X}\right),
$$

and using the numerical datum from such embedding produces families of Fano 3-folds. One approach to the classification of Fano 3-folds would be to study birational relations among these embedded Fano varieties. However, there are tens of thousands of candidate families, suggesting the impossibility of such study. Conceptually, we aim to convince the reader that only a small portion of this list may be relevant for birational classification, which could eventually result in a complete classification of Mori fibre spaces in dimension three. We give evidence that perhaps there are only a few hundreds of families that do not admit Mori fibrations over a curve or a surface. Hence, a full study of relations between those that admit only Fano structures may be possible. Next, one could continue the classification, by analysing Mori fibre spaces over a base with positive dimension.

Received 27 September 2016, accepted in final form 18 January 2017.

2010 Mathematics Subject Classification 14J10, 14J45 (primary), 14J45 (secondary).

Keywords: birational rigidity, Fano varieties, minimal model program, terminal singularities.

This journal is (C) Foundation Compositio Mathematica 2018. This article is distributed with Open Access under the terms of the Creative Commons Attribution Non-Commercial License, which permits non-commercial reuse, distribution, and reproduction in any medium, provided that the original work is properly cited. For commercial re-use, please contact the Foundation Compositio Mathematica.

The second author is partially supported by JSPS KAKENHI Grant Number 26800019. 


\section{Birationally Rigid Pfaffian FAno 3-Folds}

\subsection{Birational rigidity of Fano varieties}

A Fano variety $X$ in the Mori category, that is, $\mathbb{Q}$-factorial and terminal, is said to be birationally rigid if the only Mori fibre space birational to $X$ is $X$ itself. In other words, $X$ admits no birational structure of a strict Mori fibre space $Y \rightarrow S$ (with $\operatorname{dim} S>0$ ) and $X$ is not birational to any other Fano variety. A birationally rigid Fano variety $X$ is called birationally super-rigid if $\operatorname{Bir}(X)=\operatorname{Aut}(X)$. For example, it is known that a smooth hypersurface of degree $n$ in $\mathbb{P}^{n}$ is birationally super-rigid for $n \geqslant 4$; see [IM71, Puk98, dF13] and [Suz17] for a generalisation of this.

The first case of the example above, that is, the smooth quartic 3-folds, a celebrated result of Iskovskikh and Manin, was generalised in [CPR00] to show that a general quasi-smooth Fano hypersuface of index 1 in a weighted projective space is birationally rigid. Such a Fano variety $X$ is defined as a hypersurface $\{f=0\}$ of degree $d$ in a weighted projective space $\mathbb{P}\left(a_{0}, a_{1}, a_{2}, a_{3}, a_{4}\right)$, where $\sum a_{i}-d=1$ (hence the index), the Jacobian of $f$ vanishes only at the origin (hence quasi-smooth), and the singularities on $X$ are inherited from the ambient weighted projective space and are all terminal. There are 95 families with this property. One can consider higher codimension Fano varieties, for which the number of Fano families is shown in Table 1. These numbers currently only serve as upper bounds, except in codimensions 1, 2 and 3, where they are confirmed to be exact.

\begin{tabular}{l||c|c|c|c|c|c|c|c|c|l} 
Codimension & 1 & 2 & 3 & 4 & 5 & 6 & 7 & $\ldots$ & 18 & $\ldots$ \\
\hline Number of families & 95 & 85 & 70 & 145 & 164 & 253 & 303 & $\ldots$ & 4709 & $\ldots$
\end{tabular}

TABLE 1. Possible number of index 1 Fano families in each codimension

As mentioned before, Corti, Pukhlikov and Reid proved that a general member of each family in codimension 1 is birationally rigid [CPR00]. This was generalised by Cheltsov and Park for any such Fano variety that is quasi-smooth [CP17]. The codimension 2 families were studied by Okada in [Oka14a, Oka17, Oka14b]. For instance, the following result was shown.

Theorem 1.1 ([IP96, Oka14a]). Let $X$ be a general quasi-smooth Fano 3-fold of index 1 embedded in codimension 2 in a weighted projective space. Then $X$ is birationally rigid if and only if it belongs to one of 18 specific families.

Theorem 1.1 in particular generalises a result of Iskovskikh and Pukhlikov that shows that a general smooth complete intersection of a conic and a cubic in $\mathbb{P}^{5}$ is birationally rigid; see [IP96] and [Puk13, Chapter 2].

Theorem 1.1 has been generalised for quasi-smooth models (without the generality conditions) by Ahmadinezhad and Zucconi [AZ16].

It is crucial to note that the birationally rigid cases in Theorem 1.1 are those that do not admit a Type I centre, which is defined as follows.

Definition 1.2 (Singularity types). Let $X \subset \mathbb{P}=\mathbb{P}\left(a_{0}, \ldots, a_{n}\right)$ be a quasi-smooth Fano 3-fold. Suppose that the singular point $p \in X$ is a coordinate point of $\mathbb{P}$ of local analytic type $\frac{1}{a}(1, b, a-b)$, implying that $n-3$ of the defining polynomials of $X$ are of the form $f_{i}=x_{k}^{m} x_{i}+\cdots$, where $p$ is the $k$ th coordinate and $a=a_{k}$. Suppose that the other 3 weights (the tangent weights) are $a_{\alpha}, a_{\beta}$ and $a_{\gamma}$; then $p$ is of Type I if $(1, b, a-b)=\left(a_{\alpha}, a_{\beta}, a_{\gamma}\right)$, up to re-ordering, and $K_{X}^{3}>1 / a b(a-b)$. These are precisely the images of Type I unprojections [Rei00]. 


\section{H. Ahmadinezhad And T. OKada}

Type $\mathrm{II}_{1}$ centres are, similarly, the images of Type $\mathrm{II}_{1}$ unprojections, that is, generic complete intersections of Type II unprojections [Pap06, Pap08].

We go further and examine birational rigidity in codimension 3.

\section{Pfaffian Fano varieties}

A Pfaffian Fano 3 -fold $X$ is determined by a $5 \times 5$ skew-symmetric matrix $M$, called the syzygy matrix of $X$, whose entries are homogeneous polynomials in variables $x_{0}, \ldots, x_{6}$ with suitable weights $\operatorname{deg} x_{i}=a_{i}$. The 3 -fold $X$ is embedded in $\mathbb{P}\left(a_{0}, \ldots, a_{6}\right)$ as a codimension 3 subvariety, and it is defined by 5 Pfaffians varieties $F_{1}, \ldots, F_{5}$ of $M$. There are 69 families of Pfaffian Fano 3 -folds, which form all codimension 3 Fano 3 -folds of index 1 together with $X_{2,2,2} \subset \mathbb{P}^{6}$ (the complete intersection of 3 quadrics in $\mathbb{P}^{6}$ ). These are studied in detail in [ABR02]; they represent a success story of the application of Eisenbud-Buchsbaum structure theory of Gorenstein codimension 3 ideals [BE77]. Some explicit examples of these are scattered in this article; see, for example, Section 4.

Among these 69 families, only 5 families do not have a Type I centre. It was proved by Brown and Zucconi [BZ10] that a general Pfaffian Fano with a Type I centre is birationally non-rigid. The remaining 5 families are the main objects of this article, and the descriptions of the syzygy matrix $M$ and defining polynomials $F_{1}, \ldots, F_{5}$ will be given at the beginning of Sections 4-8 (see also the table in Section 9). Among the above 5 families, 2 families have a Type $\mathrm{II}_{1}$ centre. The aim of this article is to prove birational (super-)rigidity for the 3 families which do not admit a Type I or Type $\mathrm{II}_{1}$ centre and to prove birational non-rigidity of the 2 families which do not admit a Type I centre but admit a Type $\mathrm{II}_{1}$ centre.

Main Theorem. Let $X$ be a general Pfaffian Fano 3-fold. Then $X$ is birationally rigid if and only if it does not contain a Type $\mathrm{I}$ or Type $\mathrm{II}_{1}$ centre.

To summarise, a (general) quasi-smooth Fano variety in 95 out of 95 families in codimension 1, in 19 out of 85 families in codimension 2 and in 3 out of 70 families in codimension 3 is birationally rigid. Consequently, it is very natural to expect an affirmative answer to Question 1.3. Below (Question 1.5), we discuss a more general, and perhaps more fundamental, version of this.

Question 1.3. Does there exist a small $n$, say $n=4$ or 5 , such that for any codimension bigger than $n$ all Fano 3-folds, minimally embedded in a weighted projective space, admit a different Mori fibre space structure, that is, they are all birationally non-rigid?

\subsection{Classification of Fano 3-folds: Solid Fano varieties and Mori fibrations}

The results of [Oka17, Oka14b] go beyond birational rigidity in codimension 2 and study birigid Fano varieties in codimension 2, following [CM04]. Birigid Fanos are Mori fibre space Fano varieties that are not birationally rigid but birational to only one other Mori fibre space Fano variety. To capture this phenomenon, we introduce the following notion, which we believe will play a central role in the birational classification of Fano 3-folds.

Definition 1.4. A Fano variety is called solid if it does not admit a birational map to any strict Mori fibre space. By strict Mori fibre space we mean a Mori fibration with positive-dimensional base, that is, a Mori fibre space with Picard number strictly greater than 1 .

In particular, [Oka17] and [Oka14b] show that 6 families among the codimension 2 Fanos are non-solid (birational to del Pezzo fibrations), and the rest are expected to be solid. Following 


\section{Birationally Rigid Pfaffian Fano 3-Folds}

these observations, and based on our experience and our result on the number of rigid Fano varieties in codimension 3, we pose the following question, as a step further than Question 1.3.

Question 1.5. Do solid Fano varieties exist in higher codimensions? In other words, does there exist a small $n$ such that for any codimension bigger than $n$, all Fano 3-folds admit a structure of strict Mori fibre space?

The evidence, highlighted in this article, suggests that the answer to this question should be "no". In that case, it remains to classify solid Fano 3-folds and consider the non-solid Fano varieties as the end point of Sarkisov links on del Pezzo fibrations or conic bundles. We then examine the birational rigidity of del Pezzo fibrations and birational maps between them, and do likewise for conic bundles, a subject of further study. This will eventually give a hierarchical classification of Fano varieties and Mori fibre spaces in dimension 3.

\subsection{Notation and conventions}

We denote by $\mathrm{p}_{x_{i}}$ the vertex of $\mathbb{P}=\mathbb{P}\left(a_{0}, \ldots, a_{6}\right)$ at which only the coordinate $x_{i}$ does not vanish. For homogeneous polynomials $G_{1}, \ldots, G_{m}$, we denote by $\left(G_{1}=\cdots=G_{m}=0\right)$ the closed subscheme of $\mathbb{P}$ defined by the homogeneous ideal $\left(G_{1}, \ldots, G_{m}\right)$. For a polynomial $F$ and a monomial $g$, we write $g \in F$ if the coefficient of $g$ in $F$ is non-zero. For polynomials $f$ and $g$, we say that $f$ and $g$ are proportional (denoted $f \sim g$ ) if there are complex numbers $\lambda$ and $\mu$ with $(\lambda, \mu) \neq(0,0)$ such that $\lambda f-\mu g=0$. Let $X$ be a Pfaffian Fano 3 -fold. We always assume that $X$ is quasi-smooth, that is, its affine cone $C_{X}=\left(F_{1}=\cdots=F_{5}=0\right) \subset \mathbb{A}^{7}$, where $F_{1}, \ldots, F_{5}$ are defining polynomials of $X$, is smooth outside the origin. We set $A=-K_{X}$.

Definition 1.6. Let $X$ be a Fano 3-fold. We say that an extremal divisorial extraction $\varphi: Y \rightarrow X$ with exceptional divisor $E$ is a maximal extraction if there is a mobile linear system $\mathcal{H} \sim_{\mathbb{Q}}-n K_{X}$, where $n \in \mathbb{Q}$, such that

$$
\frac{1}{n}>c(X, \mathcal{H})=\frac{a_{E}\left(K_{X}\right)}{m_{E}(\mathcal{H})},
$$

where $c(X, \mathcal{H})=\max \left\{\lambda \mid K_{X}+\lambda \mathcal{H}\right.$ is canonical $\}$ is the canonical threshold of the pair $(X, \mathcal{H})$, $a_{E}\left(K_{X}\right)$ is the discrepancy of $K_{X}$ along $E$ and $m_{E}\left(K_{X}\right)$ is the multiplicity of $\mathcal{H}$ along $E$. The centre $\varphi(E)$ on $X$ of a maximal extraction is called a maximal centre.

Structure of the proof. The proof of the birational rigidity will be done by excluding most of the subvarieties as maximal centres and constructing a birational involution centred at the remaining subvarieties. Curves and smooth points are excluded in Section 2. Section 3 summarises the methods to exclude singular points. Then, in each following section we deal with one of the 5 families, and, finally, in Section 9, we encapsulate the results in a table.

\section{Exclusion of curves and nonsingular points}

Let $X$ be a Pfaffian Fano 3-fold. We first exclude curves as maximal centres.

Lemma 2.1. If $\left(A^{3}\right) \leqslant 1$, then no curve on $X$ is a maximal centre.

Proof. Let $\Gamma \subset X$ be an irreducible and reduced curve. We may assume that $\Gamma$ is contained in the nonsingular locus of $X$ because otherwise $\Gamma$ passes through a terminal quotient singular point and thus there is no divisorial extraction centred along $\Gamma$ (see [Kaw96]). By [Oka17, Lemma 2.9] 


\section{H. Ahmadinezhad And T. OKada}

(see also [Oka17, Remark 2.10] and [CPR00, Theorem 5.1.1]), the curve $\Gamma$ is not a maximal centre if $(A \cdot \Gamma) \geqslant\left(A^{3}\right)$. We have $(A \cdot \Gamma) \geqslant 1$ since $\Gamma$ is contained in the nonsingular locus of $X$. Thus $\Gamma$ cannot be a maximal centre since $(A \cdot \Gamma) \geqslant 1 \geqslant\left(A^{3}\right)$.

Proposition 2.2. Let $X$ be a Pfaffian Fano 3-fold without Type I centre. Then no curve on $X$ is a maximal centre.

Proof. This follows immediately from Lemma 2.1 since $\left(A^{3}\right) \leqslant 1$ in all cases.

Next, we exclude nonsingular points as maximal centres.

DeFinition 2.3. Let $X$ be a normal projective variety and $\mathrm{p} \in X$ a nonsingular point. We say that a Weil divisor class $L$ on $X$ isolates $\mathrm{p}$ if $\mathrm{p}$ is an isolated component of the base locus of the linear system

for some integer $s>0$.

$$
\mathcal{L}_{\mathrm{p}}^{s}:=\left|\mathcal{I}_{\mathrm{p}}^{s}(s L)\right|
$$

We refer the readers to [CPR00, Proof of (A), pp. 210-211] for the proof of the following lemma. The proof given there is for weighted hypersurfaces, but the same argument applies.

Lemma 2.4 ([CPR00]). Let $\mathrm{p} \in X$ be a nonsingular point of a $\mathbb{Q}$-Fano 3 -fold $X$. If $l A$ isolates $\mathrm{p}$ for some $0<l \leqslant 4 /\left(A^{3}\right)$, then $\mathrm{p}$ is not a maximal centre.

Let $\mathbb{P}:=\mathbb{P}\left(a_{0}, \ldots, a_{6}\right)$ be the weighted projective 6 -space with homogeneous coordinates $x_{0}, \ldots, x_{6}$ which is the ambient space of a Pfaffian Fano 3 -fold $X$. We assume $a_{0} \leqslant a_{1} \leqslant \cdots \leqslant a_{6}$. The following enables us to find an isolating class.

Lemma 2.5 ([CPR00, Lemma 5.6.4]). Let $\mathrm{p} \in X$ be a nonsingular point, and let $\left\{g_{i}\right\}$ be a finite set of homogeneous polynomials in variables $x_{0}, \ldots, x_{6}$. If $\mathrm{p}$ is a component of the set

$$
X \cap \bigcap\left(g_{i}=0\right),
$$

then $l A$ isolates $\mathrm{p}$, where $l=\max \left\{\operatorname{deg} g_{i}\right\}$.

Lemma 2.6. Suppose $a_{5} a_{6} \leqslant 4 /\left(A^{3}\right)$. Then no nonsingular point of $X$ is a maximal centre.

Proof. Let $\mathrm{p}=\left(\alpha_{0}: \cdots: \alpha_{6}\right) \in X$ be a nonsingular point. Then, there exists a $k \in\{0,1, \ldots, 6\}$ such that $\alpha_{k} \neq 0$. For $i=0,1, \ldots, 6$, we define

$$
m_{i}=\frac{a_{i}}{\operatorname{lcm}\left(a_{i}, a_{k}\right)} .
$$

Then we define $g_{i}=\alpha_{k}^{m_{i}} x_{i}^{m_{k}}-\alpha_{i}^{m_{k}} x_{j}^{m_{i}}$ for $i \neq k$. We have

$$
X \cap \bigcap_{i \in\{0,1, \ldots, 6\} \backslash\{k\}}\left(g_{i}=0\right)=\{\mathrm{p}\} .
$$

Moreover, we have

$$
\operatorname{deg} g_{i}=\frac{a_{i} a_{k}}{\operatorname{lcm}\left(a_{i}, a_{k}\right)} \leqslant a_{5} a_{6}
$$

for any $i \neq k$. It follows from Lemma 2.5 that $l A$ isolates $\mathrm{p}$ for some $l \leqslant a_{5} a_{6}$. Now the assumption $a_{5} a_{6} \leqslant 4 /\left(A^{3}\right)$ and Lemma 2.4 complete the proof.

Proposition 2.7. Let $X$ be a Pfaffian Fano 3-fold without Type I centre. Then no nonsingular point on $X$ is a maximal centre. 


\section{Birationally Rigid Pfaffian Fano 3-Folds}

Proof. The condition $a_{5} a_{6} \leqslant 4 /\left(A^{3}\right)$ is satisfied for Pfaffian Fano 3-folds $X$ of degrees 1/42, 1/30, $1 / 20$ and $1 / 12$. Thus the assertion for these 4 families follows from Lemma 2.6.

It remains to consider a Pfaffian Fano 3 -fold $X$ of degree $1 / 4$. Let $x, y, z_{0}, z_{1}, t_{0}, t_{1}, u$ be the homogeneous coordinates of the ambient space $\mathbb{P}\left(1,2,3^{2}, 4^{2}, 5\right)$ and $\mathrm{p} \in X$ a nonsingular point. Let $\pi: X \rightarrow \mathbb{P}:=\mathbb{P}\left(1,2,3^{2}, 4^{2}\right)$ be the projection from $\mathrm{p}_{u}$, which is indeed a morphism since $\mathrm{p}_{u} \notin X$ (see the table in Section 9). Since there are monomials $x^{12}, y^{6}, z_{0}^{4}, z_{1}^{4}, t_{0}^{3}$ and $t_{1}^{3}$ of degree 12 , we can find homogeneous polynomials $g_{1}, \ldots, g_{m}$ as suitable linear combinations of those monomials such that

$$
\bigcap\left(g_{i}=0\right)=\{\pi(\mathrm{p})\}
$$

on $\mathbb{P}$. It follows that we have

$$
X \cap \bigcap\left(g_{i}=0\right)=\pi^{-1}(\pi(\mathrm{p})),
$$

and the right-hand side consists of finitely many points including $\mathrm{p}$ since $\pi$ does not contract a curve. This shows that $12 A$ isolates $\mathrm{p}$; hence $\mathrm{p}$ cannot be a maximal centre since $12<4 /\left(A^{3}\right)=16$. This completes the proof.

\section{Excluding methods for singular points}

We will exclude singular points as maximal centres (or construct a Sarkisov link) on Pfaffian Fano 3-folds without a Type I centre in the subsequent sections. In this section, we explain the methods for excluding singular points.

We fix some notation which will be valid in the rest of this paper. Let $X$ be a Pfaffian Fano 3 -fold and $\mathrm{p} \in X$ a singular point. Let $\mathrm{p}$ be of type $\frac{1}{r}(1, a, r-a)$. We denote by $\varphi: Y \rightarrow X$ the Kawamata blowup of $X$ at $\mathrm{p}$, that is, the weighted blowup with weight $\frac{1}{r}(1, a, r-a)$. Note that $\varphi$ is the unique extremal divisorial extraction centred at the terminal quotient singular point $\mathrm{p}$ (see [Kaw96]). We denote by $E$ the exceptional divisor of $\varphi$. We set $A=-K_{X}$ and $B=-K_{Y}=\varphi^{*} A-\frac{1}{r} E$. We will frequently compute intersection numbers of divisors on $Y$. This is done by the formula

$$
\left(\varphi^{*} A^{2} \cdot E\right)=\left(\varphi^{*} A \cdot E^{2}\right)=0, \quad\left(E^{3}\right)=\frac{r^{2}}{a(r-a)} .
$$

For a curve or divisor $\Delta \subset X$, we denote by $\tilde{\Delta}$ its proper transform $\varphi_{*}^{-1} \Delta$ via $\varphi$. We will exclude singular points on $X$ by applying the following criteria.

Lemma 3.1 ([Oka17, Corollary 2.17]). If $\left(L \cdot B^{2}\right) \leqslant 0$ for some nef divisor $L$ on $Y$, then $\mathrm{p} \in X$ is not a maximal centre.

Lemma 3.2 ([Oka17, Lemma 2.18]). Assume that there are surfaces $S$ and $T$ on $Y$ with the following properties:

(i) We have $S \sim_{\mathbb{Q}} a B+d E$ and $T \sim_{\mathbb{Q}} b B+e E$ for some integers $a, b$, d, e such that $a, b>0$, $0 \leqslant e \leqslant a_{E}\left(K_{X}\right) b$ and $a e-b d \geqslant 0$.

(ii) The intersection $\Gamma:=S \cap T$ is a 1-cycle whose support consists of irreducible and reduced curves which are numerically proportional to one another.

(iii) $(T \cdot \Gamma) \leqslant 0$.

Then, $\mathrm{p} \in X$ is not a maximal extraction.

Note that in Lemma 3.2, condition (iii) is equivalent to the condition $(T \cdot S \cdot T) \leqslant 0$. 


\section{H. Ahmadinezhad And T. OKada}

When we apply Lemma 3.1, we need to find a nef divisor on $Y$, which will be done by the following result.

Lemma 3.3 ([Oka17, Lemma 6.6]). Suppose that there are prime divisors $D_{1}, \ldots, D_{k}$ on $X$ with the following properties:

(i) The intersection $D_{1} \cap \cdots \cap D_{k}$ does not contain a curve passing through $\mathrm{p}$.

(ii) For $i=1,2, \ldots, k$, the divisor $\tilde{D}_{i}$ is $\mathbb{Q}$-linearly equivalent to $b_{i} B+e_{i} E$ for some $b_{i}>0$ and $e_{i} \geqslant 0$.

(iii) We have $c \leqslant a_{E}\left(K_{X}\right)$, where $c=\max \left\{e_{i} / b_{i}\right\}$ and $a_{E}\left(K_{X}\right)$ is the discrepancy of $K_{X}$ along $E$.

Then, the divisor $L=B+c E$ is nef.

Definition 3.4. Let $X$ be a Pfaffian Fano 3-fold and $\mathrm{p} \in X$ a (singular) point. We say that $\left\{f_{1}, \ldots, f_{k}\right\}$, where $f_{1}, \ldots, f_{k}$ are homogeneous polynomials, isolates $\mathrm{p}$ if $\left(f_{1}=\cdots=f_{k}=0\right) \cap X$ does not contain a curve passing through p.

Suppose that $\left\{f_{1}, \ldots, f_{k}\right\}$ isolates a singular point $\mathrm{p} \in X$, and let $D_{i}=\left(f_{i}=0\right) \cap X$. Then $D_{1}, \ldots, D_{k}$ satisfy condition (i) of Lemma 3.3. We see

$$
\tilde{D}_{i}=b_{i} \varphi^{*} A-\operatorname{ord}_{E}\left(f_{i}\right) E=b_{i} B+\frac{b_{i}-\operatorname{rord}_{E}\left(f_{i}\right)}{r} E,
$$

where $b_{i}=\operatorname{deg} f_{i}$ and $r$ is the index of the singularity $\mathrm{p} \in X$. It follows from Lemma 3.3 that $L=B+c E$ is nef on $Y$, where

$$
c=\max \left\{\frac{b_{i}-\operatorname{rord}_{E}\left(f_{i}\right)}{b_{i} r}\right\}
$$

if $b_{i} \geqslant \operatorname{rord}_{E}\left(f_{i}\right)$ for every $i$ and $c \leqslant 1 / r$.

In the course of excluding singular points or constructing Sarkisov links, it is necessary to understand geometric objects on $Y$ (for example, proper transforms of curves or divisors on $X$ and their intersections). We will give explicit descriptions of Kawamata blowups $\varphi: Y \rightarrow X$ in terms of the embedded weighted blowup of $X \subset \mathbb{P}$ at $\mathrm{p}$ in a general setting.

From now on until the end of this section, we work in a more general setting. Let $X$ be a normal projective $\mathbb{Q}$-factorial 3 -fold defined by homogeneous polynomials $F_{1}, \ldots, F_{m} \in \mathbb{C}\left[x_{0}, \ldots, x_{n+3}\right]$ in a weighted projective space $\mathbb{P}=\mathbb{P}\left(a_{0}, \ldots, a_{n+3}\right)$ with homogeneous coordinates $x_{0}, \ldots, x_{n+3}$, and let $\mathrm{p}$ be a terminal quotient singular point of type $\frac{1}{r}(1, a, r-a)$ and $\varphi: Y \rightarrow X$ the Kawamata blowup of $X$ at $\mathrm{p}$ with exceptional divisor $E$. We give the computation of the vanishing order of a section along $E$ in the case where $\mathrm{p}$ can be transformed into a vertex by a coordinate change.

Definition 3.5. Let $\psi: V \rightarrow X$ be a birational morphism from a normal projective variety $V$ and $F$ a prime exceptional divisor of $\psi$. For a global section $s \in H^{0}\left(X, \mathcal{O}_{X}(d)\right)$, we denote by $\operatorname{ord}_{F}(s)$ the rational number such that $\psi^{*}(s=0)=\psi_{*}^{-1}(s=0)+\operatorname{ord}_{F}(s) F$ and call it the vanishing order of $s$ along $F$. For global sections $s_{1}, \ldots, s_{m}$, the expressions $\operatorname{ord}_{E}\left(s_{1}, \ldots, s_{m}\right)=$ $\frac{1}{r}\left(b_{1}, \ldots, b_{m}\right)$ and $\operatorname{ord}_{E}\left(s_{1}, \ldots, s_{m}\right) \geqslant \frac{1}{r}\left(b_{1}, \ldots, b_{m}\right)$ mean $\operatorname{ord}_{E}\left(s_{i}\right)=b_{i} / r$ and $\operatorname{ord}_{E}\left(s_{i}\right) \geqslant b_{i} / r$, respectively, for $i=1, \ldots, m$.

We assume $\mathrm{p}=\mathrm{p}_{x_{0}}$. In this case $r=a_{0}$. Then $X$ is quasi-smooth at $\mathrm{p}$ if and only if, after re-ordering $x_{1}, \ldots, x_{n+3}$ and $F_{1}, \ldots, F_{m}$, we have $x_{0}^{l_{1}} x_{1} \in F_{1}, \ldots, x_{0}^{l_{n-3}} x_{n} \in F_{n}$ for some $l_{1}, \ldots, l_{n}>0$. In this case, we have $a_{n+1} \equiv 1, a_{n+2} \equiv a, a_{n+3} \equiv r-a(\bmod r)$, after re-oredering $x_{n+1}, x_{n+2}, x_{n+3}$, and the Kawamata blowup $\varphi: Y \rightarrow X$ is the weighted blowup with weight $\mathrm{wt}\left(x_{n+1}, x_{n+2}, x_{n+3}\right)=\frac{1}{r}(1, a, r-a)$. 


\section{Birationally Rigid Pfaffian Fano 3-Folds}

We work on the open subset $U$ of $X$ where $x_{0} \neq 0$. For a polynomial $G\left(x_{0}, x_{1}, \ldots, x_{n+3}\right)$, we set $\left.G\right|_{x_{0}=1}=G\left(1, x_{1}, \ldots, x_{n+3}\right)$. Then $U$ is the geometric quotient of the affine scheme

$$
V=\left(\left.F_{1}\right|_{x_{0}=1}=\cdots=\left.F_{m}\right|_{x_{0}=1}=0\right) \subset \mathbb{A}^{n+3}
$$

by the $\mathbb{Z}_{r}$-action given by $x_{i} \mapsto \zeta^{a_{i}} x_{i}$, where $\zeta$ is a primitive $r$ th root of unity. We see that the defining polynomials $F_{n+1}, \ldots, F_{m}$ are redundant around $\mathrm{p}$ since $V$ is a local complete intersection (nonsingular) at its origin (whose image on $U$ is the point $\mathrm{p}$ ).

Definition 3.6. For a positive integer $a$, we denote by $\bar{a}$ the positive integer such that $\bar{a} \equiv a$ $(\bmod r)$ and $0<\bar{a} \leqslant r$.

We say that

$$
\mathbf{w}=\frac{1}{r}\left(b_{1}, b_{2}, \ldots, b_{n+3}\right)
$$

is an admissible weight with respect to $(X, \mathrm{p})$ if $b_{1}, \ldots, b_{6}$ are positive integer such that $b_{i} \equiv a_{i}$ $(\bmod r)$ for $i=1, \ldots, n+3$. We call

$$
\mathbf{w}_{\mathrm{in}}:=\frac{1}{r}\left(\bar{a}_{1}, \bar{a}_{2}, \ldots, \bar{a}_{n+3}\right)
$$

the initial weight of $(X, \mathrm{p})$.

Note that $\bar{r}=r$ by the above definition. Note also that the initial weight is admissible. For an admissible weight $\mathbf{w}$, we can associate the weighted blowup $\Phi_{\mathbf{w}}: Q_{\mathbf{w}} \rightarrow \mathbb{P}$ at $\mathrm{p}$ with $\operatorname{wt}\left(x_{1}, \ldots, x_{n+3}\right)=\mathbf{w}$. We see that the exceptional divisor of $\Phi_{\mathbf{w}}$ is isomorphic to the weighted projective space $\mathbb{P}\left(b_{1}, b_{2}, \ldots, b_{n+3}\right)$ with coordinates $x_{1}, \ldots, x_{n+3}$. Here, by a slight abuse of notation, we use $x_{i}$ for the coordinates of $\mathbb{P}\left(b_{1}, \ldots, b_{n+3}\right)$. In this case, $x_{i}$ has weight $b_{i}$ and this $x_{i}$ is different from the $x_{i}$ of $\mathbb{P}$. We denote by $Y_{\mathbf{w}}$ the proper transform of $X$ via $\Phi_{\mathbf{w}}$, by $\varphi_{\mathbf{w}}: Y_{\mathbf{w}} \rightarrow X$ the induced birational morphism and by $E_{\mathbf{w}}$ the exceptional divisor of $\varphi_{\mathbf{w}}$.

Definition 3.7. Let $\mathbf{w}$ be an admissible weight. For $i=1, \ldots, n$, we denote by $F_{i}^{\mathbf{w}}$ the lowestweight part of $\left.F_{i}\right|_{x_{0}=1}$ with respect to the $\mathbf{w}$-weight. We say that $\mathbf{w}$ satisfies the Kawamata blowup condition (abbreviated as KBL condition) if $x_{i} \in F_{i}^{\mathbf{w}}$ for any $i=1, \ldots, n$ and $b_{i}=\bar{a}_{i}$ for $i=n+1, n+2, n+3$ (that is, $\left.\left(b_{n+1}, b_{n+2}, b_{n+3}\right)=(1, a, r-a)\right)$.

Suppose that $\mathbf{w}$ is an admissible weight which satisfies the KBL condition. Then we have an isomorphism

$$
E_{\mathbf{w}} \cong\left(F_{1}^{\mathbf{w}}=F_{2}^{\mathbf{w}}=\cdots=F_{n}^{\mathbf{w}}=0\right) \subset \mathbb{P}\left(b_{1}, \ldots, b_{n+3}\right) .
$$

Since $x_{i} \in F_{i}^{\mathbf{w}}$ for $i=1, \ldots, n$ and $b_{n+1}=1, b_{n+2}=a, b_{n+3}=r-a$, we have an isomorphism $E_{\mathbf{w}} \cong \mathbb{P}(1, a, r-a)$ by eliminating $x_{1}, \ldots, x_{n}$. Moreover, $\varphi_{\mathbf{w}}$ is the Kawamata blowup of $X$ at $\mathrm{p}$ (see Remark 3.8).

Remark 3.8. Let $\mathbf{w}=\frac{1}{r}\left(b_{1}, \ldots, b_{n+3}\right)$ be an admissible weight satisfying the KBL condition. We now show that $\varphi_{\mathbf{w}}: Y_{\mathbf{w}} \rightarrow X$ is indeed the Kawamata blowup at $\mathrm{p}$.

The congruence condition $b_{i} \equiv a_{i}(\bmod r)$ ensures that the embedded weighted blowup of $U \subset \mathbb{A}^{n+3}$ at the origin with weight $\operatorname{wt}\left(x_{1}, \ldots, x_{n+3}\right)=\left(b_{1}, \ldots, b_{n+3}\right)$ is compatible with the $\mathbb{Z}_{r^{-}}$ action on $U \subset \mathbb{A}^{n+1}$ and gives a well-defined embedded weighted blowup of $X \subset \mathbb{P}$ at $\mathrm{p}$, which is $\varphi_{\mathbf{w}}: Y_{\mathbf{w}} \rightarrow X$. As explained above, the $\varphi_{\mathbf{w}}$-exceptional divisor $E_{\mathbf{w}}$ is isomorphic to $\mathbb{P}(1, a, r-a)$. The singular locus of $Y_{\mathbf{w}}$ along $E_{\mathbf{w}}$ is contained in the singular locus of $E_{\mathbf{w}}$. Let $\mathrm{p}_{a}$ and $\mathrm{p}_{r-a}$ be the points of $E_{\mathbf{w}}$ which correspond to the points $(0: 1: 0)$ and $(0: 0: 1)$ of $\mathbb{P}(1, a, r-a)$, respectively. Note that $E_{\mathbf{w}}$ is nonsingular outside $\left\{\mathrm{p}_{a}, \mathrm{p}_{r-a}\right\}$ and that $\mathrm{p}_{a}$ (respectively, $\mathrm{p}_{r-a}$ ) is a 


\section{H. Ahmadinezhad And T. OKada}

singular point of $E_{\mathbf{w}}$ if and only if $a>1$ (respectively, $r-a>1$ ). In view of the KBL condition, it is straightforward to check that the singularity of $Y_{\mathbf{w}}$ at $\mathrm{p}_{a}$ is of type $\frac{1}{a}(1, r-a,-1)$ when $a>1$ and that the singularity of $Y_{\mathbf{w}}$ at $\mathrm{p}_{r-a}$ is of type $\frac{1}{r-a}(1, a,-1)$ when $r-a>1$. This shows that $\varphi_{\mathbf{w}}$ is an extremal divisorial contraction centred at the terminal quotient singular point $\mathrm{p}$. By the uniqueness of such a divisorial contraction [Kaw96], we conclude that $\varphi_{\mathbf{w}}$ is indeed the Kawamata blowup at $\mathrm{p}$.

From here on, we show how to compute $\operatorname{ord}_{E}\left(x_{i}\right)$. It is clear that $\operatorname{ord}_{E}\left(x_{n+1}, x_{n+2}, x_{n+3}\right)$ $=\frac{1}{r}(1, a, r-a)$.

Lemma 3.9. Let $\mathbf{w}$ be an admissible weight satisfying the KBL condition. Then the following hold:

(i) We have $\operatorname{ord}_{E}\left(x_{i}\right) \geqslant b_{i} / r$ for $i=1, \ldots, n$.

(ii) If $F_{i}^{\mathbf{w}}$ consists only of $x_{i}$ for some $i=1, \ldots, n$, then $\operatorname{ord}_{E}\left(x_{i}\right) \geqslant\left(b_{i}+r\right) / r$.

(iii) If $F_{i}^{\mathrm{w}}$ consists only of $x_{i}$ for some $i=1, \ldots, n$, then the weight

$$
\mathbf{w}^{\prime}=\frac{1}{r}\left(b_{1}^{\prime}, \ldots, b_{n}^{\prime}, 1, a, r-a\right),
$$

where $b_{j}^{\prime}=b_{j}$ for $j \neq i$ and $b_{i}^{\prime}=b_{i}+r$, satisfies the KBL condition.

Proof. We see that $\varphi_{\mathbf{w}}$ is the Kawamata blowup of $X$ at $\mathrm{p}$ since $\mathbf{w}$ satisfies the KBL condition. It is clear that $x_{i}$ vanishes along $E_{\mathbf{w}}$ to order at least $b_{i} / r$, so that we have $\operatorname{ord}_{E}\left(x_{i}\right)=\operatorname{ord}_{E_{\mathbf{w}}}\left(x_{i}\right) \geqslant$ $b_{i} / r$. This shows statement (i).

We prove statement (iii). We have $x_{j} \in F_{j}^{\mathbf{w}}$ for $j=1, \ldots, n$ since $\mathbf{w}$ satisfies the $\mathrm{KBL}$ condition. For a monomial $g$ in the variables $x_{1}, \ldots, x_{n+3}$, the $\mathbf{w}^{\prime}$-weight of $g$ is greater than or equal to the w-weight. This implies that if there is a monomial $g \in F_{j}^{\mathbf{w}}$ whose $\mathbf{w}^{\prime}$-weight and $\mathbf{w}$-weight are the same, then $g \in F_{j}^{\mathbf{w}^{\prime}}$. If $j \neq i$, then the $\mathbf{w}$-weight and $\mathbf{w}^{\prime}$-weight of $x_{j}$ coincide, so that $x_{j} \in F_{j}^{\mathbf{w}^{\prime}}$. We have $F_{i}^{\mathbf{w}}=\alpha x_{i}$ for some $\alpha \in \mathbb{C} \backslash\{0\}$, and any other monomial in $\left.F_{i}\right|_{x_{0}=1}$ has w-weight at least $\left(b_{i}+r\right) / r$. Hence any monomial in $\left.F_{1}\right|_{x_{0}=1}$ other than $x_{i}$ has $\mathbf{w}^{\prime}$-weight at least $\left(b_{i}+r\right) / r$. Since the $\mathbf{w}^{\prime}$-weight of $x_{i}$ is $\left(b_{i}+r\right) / r$, we see $x_{i} \in F_{i}^{\mathbf{w}^{\prime}}$. This proves statement (iii). Finally, statement (ii) follows from statements (i) and (iii).

As an immediate consequence, we have the following somewhat obvious fact: $\operatorname{ord}_{E}\left(x_{i}\right) \geqslant \bar{a}_{i} / r$ for any $1 \leqslant i \leqslant n+3$.

In most of the cases, if $x_{i}$ is chosen as a general member of $H^{0}\left(X, \mathcal{O}_{X}\left(a_{i}\right)\right)$, then we have $\operatorname{ord}_{E}\left(x_{i}\right)=\bar{a}_{i} / r$. Sometimes we seek a coordinate $x_{i}$ with high vanishing order. Let us explain how to obtain such a coordinate. In general, the lowest-weight part $F_{i}^{\mathbf{w}_{\mathrm{in}}}$ with respect to the initial weight $\mathbf{w}_{\text {in }}$ contains a monomial other than $x_{i}$. Now, we suppose that, after replacing $x_{1}$ suitably, the terms in $F_{1}^{\mathbf{w}_{\text {in }}}$ other than $x_{1}$ can be eliminated, that is, $F_{1}^{\mathbf{w}_{\text {in }}}=x_{1}$. Then by Lemma 3.9, we have $\operatorname{ord}_{E}\left(x_{1}\right) \geqslant\left(\bar{a}_{1}+r\right) / r$. We can possibly repeat this process for some coordinates $x_{i}$ with $i=1,2,3$ by replacing $\mathbf{w}_{\text {in }}$ with $\mathbf{w}=\frac{1}{r}\left(\bar{a}_{1}+r, \bar{a}_{2}, \ldots, \bar{a}_{n+3}\right)$, which satisfies the KBL condition by Lemma 3.9, and we obtain coordinates $x_{i}$ which vanish along $E$ to an order higher than $\bar{a}_{i} / r$.

We will frequently apply the following simple coordinate change technique.

Lemma 3.10. Let $F$ be a polynomial of the form

$$
F=x_{0}^{3} f_{1}+x_{0}^{2}\left(\alpha x_{1}+f_{2}\right)+x_{0}\left(x_{1} f_{3}+f_{4}\right)+x_{1}^{2} f_{5}+x_{1} f_{6}+f_{7},
$$




\section{Birationally Rigid Pfaffian FAno 3-Folds}

where $\alpha \in \mathbb{C} \backslash\{0\}$ and $f_{i} \in \mathbb{C}\left[x_{2}, \ldots, x_{n}\right]$. Then, after replacing $x_{1}$ with $\gamma x_{1}+h$ for suitable $\gamma \in \mathbb{C} \backslash\{0\}$ and $h \in \mathbb{C}\left[x_{0}, x_{2}, \ldots, x_{n}\right]$, the terms divisible by $x_{0}^{2}$ in $F$ other than $\alpha x_{0}^{2} x_{1}$ are eliminated.

Proof. We may assume $\alpha=1$. Then the replacement $x_{1} \mapsto x_{1}-y f_{1}-f_{2}+f_{1} f_{3}-f_{1}^{2} f_{5}$ eliminates the terms divisible by $x_{0}^{2}$ except for $x_{0}^{2} x_{1}$.

\section{Pfaffian Fano 3-fold of degree 1/42}

Let $X=X_{16,17,18,19,20} \subset \mathbb{P}\left(1_{x}, 5_{y}, 6_{z}, 7_{t}, 8_{u}, 9_{v}, 10_{w}\right)$ be a Pfaffian Fano 3 -fold of degree $1 / 42$. Here, the degree of a Fano 3-fold means the anticanonical degree, so that $\left(A^{3}\right)=1 / 42$, where $A=-K_{X}$. We exclude all the singular points on $X$ and prove that $X$ is birationally super-rigid under a suitable generality condition. The syzygy matrix of $X$ and the defining polynomials are given as follows:

$$
M=\left(\begin{array}{ccccc}
0 & a_{6} & a_{7} & a_{8} & a_{9} \\
& 0 & b_{8} & b_{9} & b_{10} \\
& & 0 & c_{10} & c_{11} \\
& & & 0 & d_{12} \\
& & & & 0
\end{array}\right), \begin{aligned}
& F_{1}=a_{6} c_{10}-a_{7} b_{9}+a_{8} b_{8}, \\
& F_{2}=a_{6} c_{11}-a_{7} b_{10}+a_{9} b_{8}, \\
& F_{3}=a_{6} d_{12}-a_{8} b_{10}+a_{9} b_{9}, \\
& F_{4}=a_{7} d_{12}-a_{8} c_{11}+a_{9} c_{10}, \\
& F_{5}=b_{8} d_{12}-b_{9} c_{11}+b_{10} c_{10} .
\end{aligned}
$$

Here, the entries $a_{i}, b_{i}, c_{i}, d_{i}$ of $M$ are homogeneous polynomials of (weighted) degree $i$. The basket of singularities of $X$, which indicates the numbers and types of singularities, is as follows:

$$
\left\{\frac{1}{2}(1,1,1), \frac{1}{3}(1,1,2), \frac{1}{5}(1,1,4), \frac{1}{5}(1,2,3), \frac{1}{7}(1,1,6)\right\} .
$$

The aim of this section is to prove the following theorem, which will follow from Propositions 2.2 and 2.7 and the results of the present section (see also [Oka17, Theorem 2.32]). The condition in the statement will be introduced later.

Theorem 4.1. Let $X$ be a Pfaffian Fano 3-fold of degree 1/42. If $X$ satisfies Condition 4.5, then it is birationally super-rigid.

\subsection{Exclusion of the $\frac{1}{2}(1,1,1)$-point}

Lemma 4.2. The point of type $\frac{1}{2}(1,1,1)$ is not a maximal centre.

Proof. Let $\mathrm{p}$ be the point of type $\frac{1}{2}(1,1,1)$. It is clear that the set $\{x, y, t, v\}$ isolates the point $\mathrm{p}$ and $\operatorname{ord}_{E}(x, y, t, v) \geqslant \frac{1}{2}(1,1,1,1)$. Thus, we see that $L=9 \varphi^{*} A-\frac{1}{2} E$ is nef by Lemma 3.3 and we compute

$$
\left(L \cdot B^{2}\right)=9\left(A^{3}\right)-\frac{1}{2^{3}}\left(E^{3}\right)=\frac{9}{42}-\frac{1}{2}<0 .
$$

Therefore, $\mathrm{p}$ is not a maximal centre by Lemma 3.1 .

\subsection{Exclusion of the $\frac{1}{3}(1,1,2)$-point}

Lemma 4.3. The point of type $\frac{1}{3}(1,1,2)$ is not a maximal centre.

Proof. Let $\mathrm{p}$ be the point of type $\frac{1}{3}(1,1,2)$. We set $\Pi=(x=y=t=u=0)$. Then $\left.F_{5}\right|_{\Pi}=\alpha w^{2}$ with $\alpha \neq 0$ since $X$ does not contain $\mathrm{p}_{w}$. It follows that

$$
\Pi \cap X=(x=y=t=u=w=0) \cap X=\{\mathrm{p}\}
$$




\section{H. Ahmadinezhad And T. OKada}

and $\{x, y, t, u\}$ isolates $\mathrm{p}$. We see $\operatorname{ord}_{E}(x, y, t, u) \geqslant \frac{1}{3}(1,2,1,2)$. It follows that $L=7 \varphi^{*} A-\frac{1}{3} E$ is nef by Lemma 3.3 and we compute

$$
\left(L \cdot B^{2}\right)=7\left(A^{3}\right)-\frac{1}{3^{3}}\left(E^{3}\right)=\frac{7}{42}-\frac{1}{6}=0 .
$$

Therefore, $\mathrm{p}$ is not a maximal centre by Lemma 3.1 .

\subsection{Exclusion of the $\frac{1}{7}(1,1,6)$-point}

Lemma 4.4. The point of type $\frac{1}{7}(1,1,6)$ is not a maximal centre.

Proof. We claim that $\{x, y, z\}$ isolates the point $\mathrm{p}=\mathrm{p}_{t}$ of type $\frac{1}{7}(1,1,6)$. Set $\Pi=(x=y=z=$ $0)$. Then we have

$$
\left.F_{1}\right|_{\Pi}=\alpha v t+\beta u^{2},\left.\quad F_{3}\right|_{\Pi}=\gamma w u+\delta v^{2},\left.\quad F_{5}\right|_{\Pi}=\varepsilon w^{2}
$$

for some $\alpha, \beta, \ldots, \varepsilon \in \mathbb{C}$. We see that none of $\beta, \delta, \varepsilon$ is zero since $\mathrm{p}_{w}, \mathrm{p}_{v}, \mathrm{p}_{u} \notin X$. It follows that

$$
\Pi \cap X \subset(x=y=z=u=v=w=0)=\{\mathrm{p}\},
$$

that is, $\{x, y, z\}$ isolates $\mathrm{p}$. We see $\operatorname{ord}_{E}(x, y, z) \geqslant \frac{1}{7}(1,5,6)$, so that $L=\varphi^{*} A-\frac{1}{7} E$ is nef by Lemma 3.3. We compute

$$
\left(L \cdot B^{2}\right)=\left(B^{3}\right)=\left(A^{3}\right)-\frac{1}{7^{3}}\left(E^{3}\right)=\frac{1}{42}-\frac{1}{42}=0 .
$$

Therefore, $\mathrm{p}$ is not a maximal centre by Lemma 3.1 .

\subsection{Exclusion of the $\frac{1}{5}(1,1,4)$-point}

Let $\mathrm{p}$ be the point of type $\frac{1}{5}(1,1,4)$. After replacing coordinates, we may assume $\mathrm{p}=\mathrm{p}_{z}$. We see $u^{2} \in F_{1}, z^{3}, v^{2} \in F_{3}$ and $w^{2} \in F_{5}$ since $\mathrm{p}_{z}, \mathrm{p}_{u}, \mathrm{p}_{v}, \mathrm{p}_{w} \notin X$, and this implies $z \in a_{6}, z^{2} \in d_{12}$, $u \in a_{8}, b_{8}, v \in a_{9}, b_{9}$ and $w \in b_{10}, c_{10}$. We claim $t \in a_{7}$. Indeed, if $t \notin a_{7}$, then $t w \notin F_{2}$ and this implies that $X$ is not quasi-smooth at the $\frac{1}{7}(1,1,6)$-point $\mathrm{p}_{t}$. This shows $t \in a_{7}$. Moreover, since $\mathrm{p}$ is of type $\frac{1}{5}(1,1,4)$, we have $y^{2} z \notin F_{1}$, which implies $y^{2} \notin c_{10}$. By the quasi-smoothness of $X$ at $\mathrm{p}$, we have $y^{2} u \in F_{3}$, which implies $y^{2} \in b_{10}$. By setting $\Pi=(x=w=0)$ and re-scaling coordinates, we can write the restrictions of the syzygy matrix and defining polynomials to $\Pi$ as follows:

$$
\left.M\right|_{\Pi}=\left(\begin{array}{ccccc}
0 & z & t & \alpha u & \beta v \\
& 0 & u & v & y^{2} \\
& 0 & 0 & \gamma z y \\
& & 0 & \delta z^{2}+\varepsilon t y \\
& & & 0
\end{array}\right), \begin{aligned}
& \left.F_{1}\right|_{\Pi}=-t v+\alpha u^{2}, \\
& \left.F_{2}\right|_{\Pi}=\gamma z^{2} y-t y^{2}+\beta v u, \\
& \left.F_{3}\right|_{\Pi}=\delta z^{3}+\varepsilon t z y-\alpha u y^{2}+\beta v^{2}, \\
& \left.F_{4}\right|_{\Pi}=\delta t z^{2}+\varepsilon t^{2} y-\alpha \gamma u z y, \\
& \left.F_{5}\right|_{\Pi}=\delta u z^{2}+\varepsilon u t y-\gamma v z y,
\end{aligned}
$$

where $\alpha, \beta, \delta \in \mathbb{C} \backslash\{0\}$ and $\gamma, \varepsilon \in \mathbb{C}$. By the quasi-smoothness of $X$ at the $\frac{1}{7}(1,1,6)$-point $\mathrm{p}_{t}$, we have $t^{2} y \in F_{4}$, which implies $\varepsilon \neq 0$. We set $S=(x=0) \cap X$ and $T=(w=0) \cap X$. Then $\Gamma:=S \cap T$ is defined by the equations $\left.F_{1}\right|_{\Pi}=\cdots=\left.F_{5}\right|_{\Pi}=0$. We see $\operatorname{ord}_{E}(x, z, t, u, v, w) \geqslant$ $\mathbf{w}_{\text {in }}=\frac{1}{5}(1,1,2,3,4,5)$. Note that $y^{3} x^{2}, y^{2} t, y^{2} z x$ and $y^{2} z^{2}$ are the monomials of degree 17 whose initial weight is $2 / 5$. The coefficients of $t y^{2}$ and $z^{2} y$ in $F_{2}$ are -1 and $\gamma$, respectively. Let $\lambda$ and $\mu$ be the coefficients of $y^{3} x^{2}$ and $y^{2} z x$ in $F_{2}$, respectively. We define $g=-t y+\gamma z^{2}+\lambda y^{2} x^{2}+\mu y z x$. Then we can write $F_{2}=y g+G$, where each monomial in $G$ vanishes along $E$ to order at least 7/5; hence $\operatorname{ord}_{E}(g) \geqslant 7 / 5$. We set $s=\left.g\right|_{\Pi}=-t y+\gamma z^{2}$, so that we have $\left.F_{2}\right|_{\Pi}=y s+\beta u v$. 


\section{Birationally Rigid Pfaffian Fano 3-Folds}

Condition 4.5. Under the above choice of coordinates, $\gamma \neq 0$ and $\delta+\gamma \varepsilon \neq 0$.

Lemma 4.6. If $X$ satisfies Condition 4.5, then $\mathrm{p}$ is not a maximal centre.

Proof. We will show that $\{x, w, g\}$ isolates $\mathrm{p}$ or, equivalently, $\{x, w, s\}$ isolates $\mathrm{p}$. We set $\Sigma=$ $(x=w=s=0) \cap X=X \cap \Pi \cap(s=0)$. We see $v u=0$ on $\Sigma$ since $\left.F_{2}\right|_{\Pi}=y s+\beta v u$ and $\beta \neq 0$. By the equation $\left.F_{1}\right|_{\Pi}=0$ and the inequality $\alpha \neq 0$, the equality $v=0$ implies $u=0$; hence

$$
\Sigma=\left(x=w=s=u=t v=\delta z^{3}+\varepsilon t z y+\beta v^{2}=\delta t z^{2}+\varepsilon t^{2} y=\gamma v z y=0\right),
$$

set-theoretically. By the assumption $\delta+\gamma \varepsilon \neq 0$, we have that $s=-t y+\gamma z^{2}$ is not proportional to $\delta z^{2}+\varepsilon t y$, so that $\left(s=\delta z^{2}+\varepsilon t y=0\right)=(z=t y=0)$. Hence, it is straightforward to see $\Sigma=\left\{\mathrm{p}_{y}, \mathrm{p}_{t}\right\}$, which shows that $\{x, w, g\}$ isolates $\mathrm{p}$.

We have $\operatorname{ord}_{E}(x, w, s) \geqslant \frac{1}{5}(1,5,7)$, so that $L=10 \varphi^{*} A-\frac{5}{5} E$ is nef by Lemma 3.3, and we compute

$$
\left(L \cdot B^{2}\right)=10\left(A^{3}\right)-\frac{5}{5^{3}}\left(E^{3}\right)=\frac{10}{42}-\frac{1}{4}<0 .
$$

Therefore, $\mathrm{p}$ is not a maximal centre by Lemma 3.1 .

\subsection{Exclusion of the $\frac{1}{5}(1,2,3)$-point}

Let $\mathrm{p}$ be the point of type $\frac{1}{5}(1,2,3)$. We may assume $\mathrm{p}=\mathrm{p}_{y}$. By the same argument as in the previous subsection, we have $t \in a_{7}, u \in a_{8}, b_{8}, v \in a_{9}, b_{9}$ and $w \in b_{10}, c_{10}$. Since $\mathrm{p}$ is of type $\frac{1}{5}(1,2,3)$, we have $v y^{2} \in F_{4}, w y^{2} \in F_{5}$ and $t y^{2} \notin F_{2}, u y^{2} \notin F_{3}$. We see that $v y^{2} \in F_{4}$ implies $y^{2} \in c_{10}$ and $t y^{2} \notin F_{2}$ implies $y^{2} \notin b_{10}$. Since $\mathrm{p}_{t} \in X$ is of type $\frac{1}{7}(1,1,6)$, we have $t^{2} y \in F_{4}$, which implies $t y \in d_{12}$. Moreover, we have $z^{3} \in F_{3}$ since $\mathrm{p}_{z} \notin X$, which implies $z \in a_{6}$. Hence $y^{2} z \in F_{1}$. By Lemma 3.10, we can assume that $y^{2} z$ is the unique monomial in $F_{1}$ that is divisible by $y^{2}$ after replacing $z$.

We set $S=(x=0) \cap X, T=(z=0) \cap X, \Gamma=S \cap T$ and $\Pi=(x=z=0)$. Then, the restrictions of the syzygy matrix and the defining polynomials to $\Pi$ can be written as follows:

$$
\left.M\right|_{\Pi}=\left(\begin{array}{ccccc}
0 & 0 & t & \alpha u & \beta v \\
& 0 & u & v & w \\
& 0 & \gamma w+\delta y^{2} & 0 \\
& & 0 & t y \\
& & & 0
\end{array}\right), \quad \begin{aligned}
& \left.F_{1}\right|_{\Pi}=-t v+\alpha u^{2}, \\
& \left.F_{2}\right|_{\Pi}=-t w+\beta u v, \\
& \left.F_{3}\right|_{\Pi}=-\alpha u w+\beta v^{2}, \\
& \left.F_{4}\right|_{\Pi}=t^{2} y+\beta \gamma w v+\beta \delta v y^{2}, \\
& \left.F_{5}\right|_{\Pi}=u t y+\gamma w^{2}+\delta w y^{2} .
\end{aligned}
$$

Note that $\Gamma$ is defined in $\Pi$ by the above 5 polynomials. Note also that none of $\alpha, \beta, \gamma$ and $\delta$ is zero.

Lemma 4.7. The intersection $\Gamma$ is an irreducible and reduced curve.

Proof. By setting $t=1$, we work on the open subset $U \subset X$ on which $t \neq 0$. By the equations $\left.F_{1}\right|_{\Pi}=\left.F_{2}\right|_{\Pi}=0$, we can eliminate $v=\alpha u^{2}$ and $w=\beta u v=\alpha \beta u^{3}$. Hence $\Gamma \cap U$ is isomorphic to the quotient of

$$
\left(y+\alpha^{2} \beta^{2} \gamma u^{5}+\alpha \beta \delta u^{2} y^{2}=0\right) \subset \mathbb{A}_{y, u}^{2}
$$

under the natural $\mathbb{Z}_{7}$-action. Thus $\Gamma \cap U$ is an irreducible and reduced affine curve. We have $\Gamma \cap(t=0)=\{\mathrm{p}\}$. This shows that $\Gamma$ is irreducible and reduced.

By our choice of coordinates, $y^{2} z$ is the unique monomial in $F_{1}$ divisible by $y^{2}$, and we see that any monomial of degree 16 which is not divisible by $y^{2}$ has initial weight at least $6 / 5$. It 


\section{H. Ahmadinezhad And T. OKada}

follows that $\operatorname{ord}_{E}(z) \geqslant 6 / 5$ and $\varphi$ is realized as the embedded weighted blowup at $\mathrm{p}$ with weight $\operatorname{wt}(x, z, t, u, v, w)=\frac{1}{5}(1,6,2,3,4,5)=\mathrm{w}$. By looking at the monomials in $\left.F_{1}\right|_{\Pi},\left.F_{4}\right|_{\Pi},\left.F_{5}\right|_{\Pi}$, we see that the lowest-weight parts of $\left.F_{1}\right|_{y=1},\left.F_{4}\right|_{y=1}$ and $\left.F_{5}\right|_{y=1}$ are of the form

$$
F_{1}^{\mathbf{w}}=z+v t+u^{2}+f, \quad F_{4}^{\mathbf{w}}=v+t^{2}+g, \quad F_{5}^{\mathbf{w}}=w+u t+h,
$$

where $f, g, h \in \mathbb{C}[x, z, t, u, v, w]$ vanish along $(x=z=0)$. Thus we have an isomorphism

$$
E \cong\left(z+v t+f=v+t^{2}+g=w+u t+h=0\right) \subset \mathbb{P}\left(1_{x}, 6_{z}, 2_{t}, 3_{u}, 4_{v}, 5_{w}\right) .
$$

Lemma 4.8. The singular point of type $\frac{1}{5}(1,2,3)$ is not a maximal centre.

Proof. We claim $\tilde{S} \cap \tilde{T}=\tilde{\Gamma}$. To see this, it is enough to see that $\tilde{S} \cap \tilde{T}$ does not contain a curve on $E$. The lifts of the sections $x$ and $z$ on $Y$ restrict, respectively, to the coordinates $x$ and $z$ of the ambient weighted projective space of $E$, and their zero loci coincide with $\tilde{S} \cap E$ and $\tilde{T} \cap E$, respectively. Since $f, g, h$ are in the ideal $(x, z)$, the set

$$
\tilde{S} \cap \tilde{T} \cap E=\left(x=z=v t+u^{2}=v+t^{2}=w+u t=0\right)
$$

consists of a single point. Thus $\tilde{S} \cap \tilde{T}=\tilde{\Gamma}$. Since $\tilde{S} \sim_{\mathbb{Q}} \varphi^{*} A-\frac{1}{5} E$ and $\tilde{T} \sim_{\mathbb{Q}} 6 \varphi^{*} A-\frac{6}{5} E$, we have

$$
(\tilde{T} \cdot \tilde{S} \cdot \tilde{T})=6^{2}\left(A^{3}\right)-\frac{6^{2}}{5^{3}}\left(E^{3}\right)=\frac{6}{7}-\frac{6}{5}<0 .
$$

Therefore, $\mathrm{p}$ is not a maximal centre by Lemma 3.2.

\section{Pfaffian Fano 3-fold of degree 1/30}

Let $X=X_{14,15,16,17,18} \subset \mathbb{P}\left(1_{x}, 5_{y_{0}}, 5_{y_{1}}, 6_{z}, 7_{t}, 8_{u}, 9_{v}\right)$ be a Pfaffian Fano 3 -fold of degree $1 / 30$. We exclude all the singular points on $X$ and prove that $X$ is birationally super-rigid under a suitable generality condition. The syzygy matrix of $X$ and the defining polynomials are given as follows:

$$
M=\left(\begin{array}{ccccc}
0 & a_{5} & a_{6} & a_{7} & a_{8} \\
& 0 & b_{7} & b_{8} & b_{9} \\
& & 0 & c_{9} & c_{10} \\
& & & 0 & d_{11} \\
& & & & 0
\end{array}\right), \begin{aligned}
& F_{1}=a_{5} c_{9}-a_{6} b_{8}+a_{7} b_{7}, \\
& F_{2}=a_{5} c_{10}-a_{6} b_{9}+a_{8} b_{7}, \\
& F_{3}=a_{5} d_{11}-a_{7} b_{9}+a_{8} b_{8}, \\
& F_{4}=a_{6} d_{11}-a_{7} c_{10}+a_{8} c_{9}, \\
& F_{5}=b_{7} d_{11}-b_{8} c_{10}+b_{9} c_{9} .
\end{aligned}
$$

The basket of singularities of $X$ is

$$
\left\{\frac{1}{5}(1,1,4), 2 \times \frac{1}{5}(1,2,3), \frac{1}{6}(1,1,5)\right\} .
$$

The aim of this section is to prove the following theorem, which will follow from Propositions 2.2 and 2.7 and the results of the present section. The condition in the statement will be introduced later.

Theorem 5.1. Let $X$ be a Pfaffian Fano 3-fold of degree 1/30. If $X$ satisfies Condition 5.6, then it is birationally super-rigid.

\subsection{Exclusion of the $\frac{1}{5}(1,2,3)$-points}

Let $\mathrm{p}$ be a point of type $\frac{1}{5}(1,2,3)$. After replacing $y_{0}$ and $y_{1}$, we may assume $\mathrm{p}=\mathrm{p}_{y_{1}}$. Note that this implies $y_{1}^{3} \notin F_{2}$. Note also that $t^{2} \in F_{1}, u^{2} \in F_{3}$ and $v^{2} \in F_{5}$ since $\mathrm{p}_{t}, \mathrm{p}_{u}, \mathrm{p}_{v} \notin X$, which 


\section{Birationally Rigid Pfaffian Fano 3-Folds}

implies $t \in a_{7}, b_{7}, u \in a_{8}, b_{8}$ and $v \in b_{9}, c_{9}$. By the quasi-smoothness of $X$ at $\mathrm{p}$, we have $y_{1} v \in F_{1}$ and $y_{1}^{2} y_{0} \in F_{2}$. We divide the proof into 2 cases according to whether $y_{1}^{2} z \in F_{3}$ or not.

First, we treat the case where $y_{1}^{2} z \in F_{3}$.

Lemma 5.2. If $y_{1}^{2} z \in F_{3}$, then $\mathrm{p}$ is not a maximal centre.

Proof. Recall that $y_{1} v \in F_{1}, y_{1}^{2} y_{0} \in F_{2}$ and $y_{1}^{2} z \in F_{3}$. By Lemma 3.10, we may assume that $y_{1}^{2} z$ is the unique monomial in $F_{3}$ divisible by $y_{1}^{2}$. Consider the weight $\operatorname{wt}\left(x, y_{0}, z, t, u, v\right)=$ $\frac{1}{5}(1,5,6,2,3,4)=: \mathbf{w}$. Then $v \in F_{1}^{\mathbf{w}}, y_{0} \in F_{2}^{\mathbf{w}}$ and $z \in F_{3}^{\mathbf{w}}$, so that $\varphi$ is realized as the embedded weighted blowup at $\mathrm{p}$ with the weight $\mathbf{w}$.

We claim that $\left\{x, y_{0}, z\right\}$ isolates $\mathrm{p}$. Set $\Pi=\left(x=y_{0}=z=0\right)$. We have

$$
\left.F_{1}\right|_{\Pi}=t^{2}+\alpha v y_{1},\left.\quad F_{3}\right|_{\Pi}=u^{2}+\beta v t,\left.\quad F_{5}\right|_{\Pi}=v^{2}+\gamma u y_{1}^{2},
$$

for some $\alpha, \beta, \gamma \in \mathbb{C}$. Hence

$$
\left(x=y_{0}=z=0\right) \cap X \subset\left(x=y_{0}=z=\left.F_{1}\right|_{\Pi}=\left.F_{3}\right|_{\Pi}=\left.F_{5}\right|_{\Pi}=0\right),
$$

and it is straightforward to see that the set on the right-hand side is finite (for any $\alpha, \beta, \gamma$ ). This shows that $\left\{x, y_{0}, z\right\}$ isolates $\mathrm{p}$. We see $\operatorname{ord}_{E}\left(x, y_{0}, z\right) \geqslant \frac{1}{5}(1,5,6)$, so that $L:=B$ is nef by Lemma 3.3 and we compute

$$
\left(L \cdot B^{2}\right)=\left(B^{3}\right)=\left(A^{3}\right)-\frac{1}{5^{3}}\left(E^{3}\right)=\frac{1}{30}-\frac{1}{30}=0 .
$$

Therefore, $\mathrm{p}$ is not a maximal centre by Lemma 3.1.

Next, we treat the case where $y_{1}^{2} z \notin F_{3}$. In this case, we have $y_{1}^{3} x \in F_{3}$. We set $S=\left(x_{0}=\right.$ $0) \cap X, T=\left(y_{0}=0\right) \cap X$ and $\Gamma=S \cap T$.

Lemma 5.3. The support of $\Gamma$ is an irreducible curve.

Proof. We set $\Pi=\left(x_{0}=y_{0}=0\right)$. We have $y_{1} \in a_{5}$ because otherwise $F_{3}=a_{5} d_{11}-a_{7} b_{9}+a_{8} b_{8}$ cannot contain $y_{1}^{3} x$. Then, we see $y_{1}^{2} \notin c_{10}$ since $y_{1}^{3} \notin F_{2}$. Note also that $z y_{1} \notin d_{11}$ since $y_{1}^{2} z \notin F_{3}$. We can write the restrictions of the syzygy matrix and defining polynomials to $\Pi$ as

$$
\left.M\right|_{\Pi}=\left(\begin{array}{ccccc}
0 & y_{1} & \alpha z & \beta t & \gamma u \\
& 0 & t & u & \delta v \\
& 0 & v & 0 \\
& & 0 & 0 \\
& & & & 0
\end{array}\right), \quad \begin{aligned}
& \left.F_{1}\right|_{\Pi}=y_{1} v-\alpha z u+\beta t^{2}, \\
& \left.F_{2}\right|_{\Pi}=-\alpha \delta z v+\gamma u t, \\
& \left.F_{3}\right|_{\Pi}=-\beta \delta v t+\gamma u^{2}, \\
& \left.F_{4}\right|_{\Pi}=\gamma \delta u v \\
& \left.F_{5}\right|_{\Pi}=\delta v^{2} .
\end{aligned}
$$

Note that $\Gamma=X \cap \Pi$ is defined in $\Pi$ by the above 5 polynomials. Since $\beta, \gamma, \delta \neq 0$, we have $\Gamma=(t=u=v=0) \cap \Pi$ set-theoretically and the proof is completed.

LEMmA 5.4. If $y_{1}^{2} z \notin F_{3}$, then $\mathrm{p}$ is not a maximal centre.

Proof. We will show that the support of $\tilde{S} \cap \tilde{T}$ is the proper transform of the support of $S \cap T$. Consider the weight $\operatorname{wt}\left(x, y_{0}, z, t, u, v\right)=\frac{1}{5}(6,5,1,2,3,4)=: \mathbf{w}$. Then $v \in F_{1}^{\mathbf{w}}, y_{0} \in F_{2}^{\mathbf{w}}$ and $x \in F_{3}^{\mathbf{w}}$ since $F_{3}$ does not contain $y_{1}^{2} z$, which is the unique monomial of degree 16 with wweight $\frac{1}{5}$. It follows that $\varphi$ is realized as the embedded weighted blowup at $\mathrm{p}$ with weight $\mathbf{w}$, and we have an isomorphism

$$
E \cong\left(F_{1}^{\mathbf{w}}=F_{2}^{\mathbf{w}}=F_{3}^{\mathbf{w}}=0\right) \subset \mathbb{P}\left(6_{x}, 5_{y_{0}}, 1_{z}, 2_{t}, 3_{u}, 4_{v}\right) .
$$




\section{H. Ahmadinezhad And T. OKada}

In view of the description of $\left.F_{1}\right|_{\Pi},\left.F_{2}\right|_{\Pi}$ and $\left.F_{3}\right|_{\Pi}$, after re-scaling $t$ and $u$, we can write

$$
F_{1}^{\mathrm{w}}=v+\alpha z u+t^{2}+f, \quad F_{2}^{\mathrm{w}}=x+\beta v z+\gamma u t+g, \quad F_{3}^{\mathbf{w}}=y_{0}+\delta v t+u^{2}+h,
$$

where $\alpha, \ldots, \delta \in \mathbb{C}$ with $\gamma, \delta \neq 0$ and $f, g, h$ are contained in the ideal $\left(x, y_{0}\right)$ (Note that $x \notin g$ and $\left.y_{0} \notin h\right)$. We have

$$
\tilde{S} \cap \tilde{T} \cap E=\left(x=y_{0}=0\right) \cap E=\left(x=y_{0}=v+\alpha z u+t^{2}=\beta v z+\gamma u t=\delta v t+u^{2}=0\right),
$$

and this is a finite set of points since $\gamma, \delta \neq 0$. Thus, $\tilde{\Gamma} \cap \tilde{S}$ is the proper transform of $S \cap T$.

We have $\tilde{S} \sim_{\mathbb{Q}} \varphi^{*} A-\frac{6}{5} E$ and $\tilde{T} \sim_{\mathbb{Q}} 5 \varphi^{*} A-\frac{5}{5} E$, so that

$$
(\tilde{T} \cdot \tilde{S} \cdot \tilde{T})=5^{2}\left(A^{3}\right)-\frac{5^{2} \cdot 6}{5^{3}}\left(E^{3}\right)=\frac{5}{6}-5<0 .
$$

Therefore, $\mathrm{p}$ is not a maximal centre by Lemma 3.2 .

\subsection{Exclusion of the $\frac{1}{6}(1,1,5)$-point}

Lemma 5.5. The point of type $\frac{1}{6}(1,1,5)$ is not a maximal centre.

Proof. We claim that $\left\{x, y_{0}, y_{1}\right\}$ isolates the $\frac{1}{6}(1,1,5)$-point $\mathrm{p}=\mathrm{p}_{z}$. Set $\Pi=\left(x=y_{0}=y_{1}=0\right)$. Then, we can write

$$
\left.F_{1}\right|_{\Pi}=\alpha u z+\beta t^{2},\left.\quad F_{3}\right|_{\Pi}=\gamma v t+\delta u^{2},\left.\quad F_{5}\right|_{\Pi}=\varepsilon v^{2}
$$

for some $\alpha, \beta, \ldots, \varepsilon \in \mathbb{C}$. Moreover, none of $\beta, \delta, \varepsilon$ is zero since $\mathrm{p}_{t}, \mathrm{p}_{u}, \mathrm{p}_{v} \notin X$. Hence

$$
\left(x=y_{0}=y_{1}=0\right) \cap X \subset\left(x=y_{0}=y_{1}=t=u=v=0\right)=\{\mathbf{p}\} ;
$$

that is, $\left\{x, y_{0}, y_{1}\right\}$ isolates $\mathrm{p}$.

It is clear that $\operatorname{ord}_{E}\left(x, y_{0}, y_{1}\right) \geqslant \frac{1}{6}(1,5,5)$ since $x, y_{0}, y_{1}$ are of degree $1,5,5$, respectively (see Lemma 3.9(1)), so that $L=\varphi^{*} A-\frac{1}{6} E$ is nef by Lemma 3.3. We compute

$$
\left(L \cdot B^{2}\right)=\left(B^{3}\right)=\left(A^{3}\right)-\frac{1}{6^{3}}\left(E^{3}\right)=\frac{1}{30}-\frac{1}{30}=0 .
$$

Therefore, $\mathrm{p}$ is not a maximal centre by Lemma 3.1 .

\subsection{Exclusion of the $\frac{1}{5}(1,1,4)$-point}

Let $\mathrm{p} \in X$ be the point of type $\frac{1}{5}(1,1,4)$. After replacing $y_{0}$ and $y_{1}$, we may assume $\mathrm{p}=\mathrm{p}_{y_{1}}$. We have $t^{2} \in F_{1}, u^{2} \in F_{3}$ and $v^{2} \in F_{5}$ since $\mathrm{p}_{t}, \mathrm{p}_{u}, \mathrm{p}_{v} \notin X$, which implies $t \in a_{7}, b_{7}, u \in a_{8}, b_{8}$ and $v \in b_{9}, c_{9}$. Since $\mathrm{p}$ is of type $\frac{1}{5}(1,1,4)$, we have $v y_{1} \notin F_{1}$ and $y_{1}^{2} z \notin F_{3}$, which implies $y_{1} \notin a_{5}$. Since $X$ has a point of type $\frac{1}{6}(1,1,5)$ at $\mathrm{p}_{z}$, we have $v z \in F_{2}$, which implies $z \in a_{6}$. Since $X$ has a single point of type $\frac{1}{5}(1,1,4)$ and 2 distinct points of type $\frac{1}{5}(1,2,3)$, the set $\left(x=z=t=u=v=a_{5} c_{10}=0\right)$ consists of 3 distinct points. This implies $y_{1}^{2} \in c_{10}$ since $y_{1} \notin a_{5}$. We set $\Pi=\left(x_{0}=y_{0}=0\right)$. Then the restrictions of the syzygy matrix and the defining polynomials can be written as follows:

$$
\left.M\right|_{\Pi}=\left(\begin{array}{ccccc}
0 & 0 & z & \alpha t & \beta u \\
& 0 & t & u & v \\
& & 0 & \gamma v & y_{1}^{2} \\
& & 0 & \delta z y_{1}^{2} \\
& & & 0
\end{array}\right), \quad \begin{aligned}
& \left.F_{1}\right|_{\Pi}=\alpha t^{2}-z u, \\
& \left.F_{2}\right|_{\Pi}=\beta u t-z v, \\
& \left.F_{3}\right|_{\Pi}=\beta u^{2}-\alpha t v, \\
& \left.F_{4}\right|_{\Pi}=\beta \gamma u v-\alpha t y_{1}^{2}+\delta z^{2} y_{1}, \\
& \left.F_{5}\right|_{\Pi}=\gamma v^{2}-u y_{1}^{2}+\delta t z y_{1},
\end{aligned}
$$




\section{Birationally Rigid Pfaffian FAno 3-Folds}

where $\alpha, \beta, \gamma \in \mathbb{C} \backslash\{0\}$ and $\delta \in \mathbb{C}$. We set $S=\left(x_{0}=0\right) \cap X$ and $T=\left(y_{0}=0\right) \cap X$ and let $\Gamma=S \cap T$ be the scheme-theoretic intersection. We assume the following condition.

Condition 5.6. Under the above choice of coordinates, $\delta \neq 0$.

Lemma 5.7. The intersection $\Gamma$ is an irreducible and reduced curve.

Proof. The curve $\Gamma=X \cap \Pi$ is defined by $\left.F_{1}\right|_{\Pi}=\cdots=\left.F_{5}\right|_{\Pi}=0$ in $\Pi$. Recall that $\alpha, \beta, \gamma \neq 0$. We work on the open subset on which $z \neq 0$. By setting $z=1$ in $\left.F_{1}\right|_{\Pi}=\left.F_{2}\right|_{\Pi}=0$, we have $u=\alpha t^{2}$ and $v=\beta u t=\alpha \beta t^{3}$. By eliminating $u$ and $v$ in the equation $\left.F_{3}\right|_{\Pi}=\left.F_{4}\right|_{\Pi}=\left.F_{5}\right|_{\Pi}=0$, we see that, on $z \neq 0$, the curve $\Gamma$ is isomorphic to the quotient of the curve

$$
\left(\delta y_{1}-\alpha y_{1}^{2} t+\alpha^{2} \beta^{2} \gamma t^{5}=0\right) \subset \mathbb{A}_{y_{1}, t}^{2}
$$

by the natural $\mathbb{Z}_{5}$-action on $\mathbb{A}^{2}$. On the other hand, $\Gamma \cap(z=0)$ consists of the single point $\mathrm{p}$. Therefore, $\Gamma$ is an irreducible and reduced curve.

Lemma 5.8. The point of type $\frac{1}{5}(1,1,4)$ is not a maximal centre.

Proof. We have $y_{1}^{2} y_{0} \in F_{2}, y_{1}^{2} t \in F_{4}$ and $y_{1}^{2} u \in F_{5}$ by the quasi-smoothness of $X$ at $\mathrm{p}$. Consider the initial weight $\operatorname{wt}\left(x, y_{0}, z, t, u, v\right)=\frac{1}{5}(1,5,1,2,3,4)=\mathbf{w}_{\text {in }}$. In view of the description of $\left.F_{1}\right|_{\Pi}$, after re-scaling coordinates, we have

$$
F_{2}^{\mathbf{w}_{\text {in }}}=y_{0}+u t+z v+f, \quad F_{4}^{\mathbf{w}_{\text {in }}}=t+\delta_{1} z^{2}+g, \quad F_{5}^{\mathbf{w}_{\text {in }}}=u+\delta_{2} t z+h,
$$

where $\delta_{1}, \delta_{2} \in \mathbb{C} \backslash\{0\}$ and $f, g, h$ are contained in the ideal $\left(x_{0}, y_{0}\right)$ (note that $\left.y_{0} \notin f\right)$. We see that $\varphi$ is realized as the embedded weighted blowup at $\mathrm{p}$ with weight $\mathbf{w}_{\text {in }}$, and we have an isomorphism

$$
E \cong\left(F_{2}^{\mathbf{w}_{\text {in }}}=F_{4}^{\mathbf{w}_{\text {in }}}=F_{5}^{\mathbf{w}_{\text {in }}}=0\right) \subset \mathbb{P}\left(1_{x}, 5_{y_{0}}, 1_{z}, 2_{t}, 3_{u}, 4_{v}\right)
$$

We see that

$$
\tilde{S} \cap \tilde{T} \cap E=\left(x=y_{0}=u t-z v=t+\delta_{1} z^{2}=u+\delta_{2} t z=0\right)
$$

is a finite set, which imlies $\tilde{S} \cap \tilde{T}=\tilde{\Gamma}$.

We have $\tilde{S} \sim_{\mathbb{Q}} \varphi^{*} A-\frac{1}{5} E$ and $\tilde{T} \sim_{\mathbb{Q}} 5 \varphi^{*} A-\frac{5}{5} E$, so that

$$
(\tilde{T} \cdot \tilde{S} \cdot \tilde{T})=5^{2}\left(A^{3}\right)-\frac{5^{2}}{5^{3}}\left(E^{3}\right)=\frac{5}{6}-\frac{5}{4}<0 .
$$

Therefore, $\mathrm{p}$ is not a maximal centre by Lemma 3.2.

\section{Pfaffian Fano 3-fold of degree 1/20}

Let $X=X_{12,13,14,15,16} \subset \mathbb{P}\left(1_{x}, 4_{y}, 5_{z_{0}}, 5_{z_{1}}, 6_{t}, 7_{u}, 8_{v}\right)$ be a Pfaffian Fano 3 -fold of degree $1 / 20$. We exclude singular points on $X$ other than the $\frac{1}{5}(1,2,3)$-point at which there is a birational involution and prove that $X$ is birationally rigid under a suitable generality condition. The syzygy matrix of $X$ and the defining polynomials are given as follows:

$$
M=\left(\begin{array}{ccccc}
0 & a_{4} & a_{5} & a_{6} & a_{7} \\
& 0 & b_{6} & b_{7} & b_{8} \\
& & 0 & c_{8} & c_{9} \\
& & & 0 & d_{10} \\
& & & & 0
\end{array}\right), \begin{aligned}
& F_{1}=a_{4} c_{8}-a_{5} b_{7}+a_{6} b_{6}, \\
& F_{2}=a_{4} c_{9}-a_{5} b_{8}+a_{7} b_{6}, \\
& F_{3}=a_{4} d_{10}-a_{6} b_{8}+a_{7} b_{7}, \\
& F_{4}=a_{5} d_{10}-a_{6} c_{9}+a_{7} c_{8}, \\
& F_{5}=b_{6} d_{10}-b_{7} c_{9}+b_{8} c_{8} .
\end{aligned}
$$




\section{H. Ahmadinezhad And T. OKada}

The basket of singularities of $X$ is

$$
\left\{\frac{1}{2}(1,1,1), \frac{1}{4}(1,1,3), 2 \times \frac{1}{5}(1,1,4), \frac{1}{5}(1,2,3)\right\} .
$$

The aim of this section is to prove the following theorem, which will follow from Propositions 2.2 and 2.7 and the results of the present section. The condition in the statement will be introduced later.

Theorem 6.1. Let $X$ be a Pfaffian Fano 3-fold of degree 1/20. If $X$ satisfies Condition 6.4, then it is birationally rigid.

\subsection{Exclusion of the $\frac{1}{2}(1,1,1)$-point}

Lemma 6.2. The singular point of type $\frac{1}{2}(1,1,1)$ is not a maximal centre.

Proof. Let $\mathrm{p}$ be the point of type $\frac{1}{2}(1,1,1)$. It is clear that $\left\{x, z_{0}, z_{1}, u\right\}$ isolates $\mathrm{p}$ and $\operatorname{ord}_{E}\left(x, z_{0}\right.$, $\left.z_{1}, u\right) \geqslant \frac{1}{2}(1,1,1,1)$. It follows that $L=7 \varphi^{*} A-\frac{1}{2} E$ is nef by Lemma 3.3 , and we compute

$$
\left(L \cdot B^{2}\right)=7\left(A^{3}\right)-\frac{1}{2^{3}}\left(E^{3}\right)=\frac{7}{20}-\frac{1}{2}<0 .
$$

Therefore, $\mathrm{p}$ is not a maximal centre by Lemma 3.1 .

\subsection{Exclusion of the $\frac{1}{5}(1,1,4)$-points}

Lemma 6.3. A singular point of type $\frac{1}{5}(1,1,4)$ is not a maximal centre.

Proof. Let $\mathrm{p}$ be a point of type $\frac{1}{5}(1,1,4)$. We may assume $\mathrm{p}=\mathrm{p}_{z_{1}}$ after replacing $z_{0}$ and $z_{1}$. We claim that $\left\{x, y, z_{0}\right\}$ isolates $\mathrm{p}$. Set $\Pi=\left(x=y=z_{0}=0\right)$. Note that $t^{2} \in F_{1}, u^{2} \in F_{3}$ and $v^{2} \in F_{5}$ since $\mathrm{p}_{t}, \mathrm{p}_{u}, \mathrm{p}_{v} \notin X$, hence we may assume that those coefficients are 1 . Then, we can write

$$
\left.F_{1}\right|_{\Pi}=t^{2}+\alpha u z_{1},\left.\quad F_{3}\right|_{\Pi}=u^{2}+\beta v t,\left.\quad F_{5}\right|_{\Pi}=v^{2}+\gamma t z_{1}^{2}
$$

for some $\alpha, \beta, \gamma \in \mathbb{C}$. We see that

$$
\left(x=y=z_{0}=0\right) \cap X \subset\left(x=y=z_{0}=\left.F_{1}\right|_{\Pi}=\left.F_{2}\right|_{\Pi}=\left.F_{3}\right|_{\Pi}=0\right),
$$

and the set on the right-hand side of this inclusion is finite (for any $\alpha, \beta, \gamma \in \mathbb{C}$ ). This shows that $\left\{x, y, z_{0}\right\}$ isolates $\mathrm{p}$.

We see $\operatorname{ord}_{E}\left(x, y, z_{0}\right) \geqslant \frac{1}{5}(1,4,5)$, so that $L=B$ is nef by Lemma 3.3. We compute

$$
\left(L \cdot B^{2}\right)=\left(B^{3}\right)=\left(A^{3}\right)-\frac{1}{5^{3}}\left(E^{3}\right)=\frac{1}{20}-\frac{1}{20}=0 .
$$

Therefore, $\mathrm{p}$ is not a maximal centre by Lemma 3.1 .

\subsection{Exclusion of the $\frac{1}{4}(1,1,3)$-point}

Let $\mathrm{p}$ be a point of type $\frac{1}{4}(1,1,3)$. Replacing $v$, we may assume $\mathrm{p}=\mathrm{p}_{y}$. We claim $y \in a_{4}$. Indeed, if $y \notin a_{4}$, then $z_{0}^{2} y, z_{0} z_{1} y, z_{1}^{2} y \notin F_{3}$. This gives a contradiction since $X$ admits a point $\mathrm{q}$ of type $\frac{1}{5}(1,2,3)$, hence there must be at least one of $z_{0}^{2} y, z_{0} z_{1} y$ and $z_{1}^{2} y$ in $F_{3}$. Hence $y \in a_{4}$ and we may assume that the coefficient of $y$ in $a_{4}$ is 1 after re-scaling $y$. We can write $a_{5}=\ell_{1}+$ (other terms), $c_{9}=y \ell_{2}+$ (other terms) and $d_{10}=q+$ (other terms), where $\ell_{1}$ and $\ell_{2}$ are linear forms in $z_{0}$, $z_{1}$ and $q$ is the quadratic form in $z_{0}, z_{1}$. Let $\delta \in \mathbb{C}$ be the coefficient of $y^{2} \in b_{8}$. We exclude $\mathrm{p}$ assuming the following. 


\section{Birationally Rigid Pfaffian Fano 3-Folds}

Condition 6.4. Under the above choice of coordinates, the polynomials $\ell_{2}-\delta \ell_{1}$ and $q$ have no (non-trivial) common root.

We have $F_{2}=y^{2}\left(\ell_{2}-\delta \ell_{1}\right)+$ (other terms). Condition 6.4 in particular implies $\ell_{2}-\delta \ell_{1} \neq 0$. Replacing $z_{0}$ and $z_{1}$, we may assume $\ell_{2}-\delta \ell_{1}=z_{1}$. This means that $y^{2} z_{1} \in F_{2}$ and $y^{2} z_{0} \notin F_{2}$. By Lemma 3.10, replacing $z_{1}$ further, we may assume that $y^{2} z_{1}$ is the unique monomial in $F_{2}$ divisible by $y^{2}$. We have $t^{2} \in F_{1}, u^{2} \in F_{3}$ and $v^{2} \in F_{5}$ since $\mathrm{p}_{t}, \mathrm{p}_{u}, \mathrm{p}_{v} \notin X$, which implies $t \in a_{6}, b_{6}, u \in a_{7}, b_{7}$ and $v \in b_{8}, c_{8}$. By setting $\Pi=\left(x=z_{1}=0\right)$, we can write

$$
\left.M\right|_{\Pi}=\left(\begin{array}{ccccc}
0 & y & \alpha z_{0} & t & \beta u \\
& 0 & \gamma t & u & v+\delta y^{2} \\
& & 0 & \varepsilon v+\eta y^{2} & \zeta z_{0} y \\
& & & 0 & \lambda t y+\mu z_{0}^{2} \\
& & & & 0
\end{array}\right),
$$

where $\alpha, \beta, \ldots, \mu \in \mathbb{C}$. Note that $\beta, \gamma, \varepsilon \neq 0$. Note also that $\alpha$ is the coefficient of $z_{0}$ in $\ell_{1}$ and $\mu$ is the coefficient of $z_{0}^{2}$ in $q$. We have $\mu \neq 0$ because otherwise $\ell_{2}-\delta \ell_{1}=q=0$ has a solution $z_{1}=0$ and this is impossible by Condition 6.4 (here, recall that $\ell_{2}-\delta \ell_{1}=z_{1}$ ). Since $\mathrm{p}=\mathrm{p}_{y} \in X$ and the coefficient of $y^{3}$ in $\left.F_{1}\right|_{\Pi}$ is $\eta$, we have $\eta=0$. The coefficient of $y^{2} z_{0}$ in $\left.F_{2}\right|_{\Pi}$ is $\alpha(\zeta-\delta)$ which must be zero by our choice of coordinates. Thus, we have

$$
\begin{aligned}
& \left.F_{1}\right|_{\Pi}=\varepsilon y v-\alpha u z_{0}+\gamma t^{2}, \\
& \left.F_{2}\right|_{\Pi}=\beta \gamma u t-\alpha v z_{0}, \\
& \left.F_{3}\right|_{\Pi}=(\lambda-\delta) t y^{2}+\mu z_{0}^{2} y-v t+\beta u^{2}, \\
& \left.F_{4}\right|_{\Pi}=(\alpha \lambda-\zeta) t z_{0} y+\alpha \mu z_{0}^{3}+\beta \varepsilon v u, \\
& \left.F_{5}\right|_{\Pi}=\gamma \lambda t^{2} y+\gamma \mu t z_{0}^{2}-\zeta u z_{0} y+\varepsilon v^{2}+\delta \varepsilon v y^{2} .
\end{aligned}
$$

By the quasi-smoothness of $X$ at $\mathrm{p}$, we have $\lambda-\delta \neq 0$. We compute $\operatorname{ord}_{E}\left(z_{1}\right)$. We see that $y^{3} x$, $y^{2} z_{0}$ and $y^{2} z_{1}$ are the monomials of degree 13 which have initial weight $1 / 4$ and $y^{3} x, y^{2} z_{0} \notin F_{2}$ by our choice of coordinates, hence $\operatorname{ord}_{E}\left(z_{1}\right) \geqslant 5 / 4$. It follows that $\varphi$ is realized as the embedded weighted blowup at $\mathrm{p}$ with $\mathrm{wt}\left(x, z_{0}, z_{1}, t, u, v\right)=\frac{1}{4}(1,1,5,2,3,4)=: \mathbf{w}$.

We first consider the general case $\alpha \neq 0$. Set $S=(x=0) \cap X, T=\left(z_{1}=0\right) \cap X$ and $\Gamma=S \cap T=\Pi \cap X$.

Lemma 6.5. If $\alpha \neq 0$, then $\Gamma$ is an irreducible and reduced curve.

Proof. In this case, we have $\zeta=\delta$ since $\alpha(\zeta-\delta)=0$. We work on the open subset $U=\left(z_{0} \neq 0\right) \subset$ $\Pi$ by setting $z_{0}=1$. Re-scaling $z_{0}$, we may assume $\alpha=1$. By $\left.F_{2}\right|_{\Pi}=0$, we have $v=\beta \gamma u t$. For a polynomial $F=F\left(x, y, z_{0}, z_{1}, t, u, v\right)$, we set $\bar{F}=F(0, y, 1,0, u, \beta \gamma u t)$. Then, by eliminating $v$, we see that $\Gamma \cap U$ is the quotient of affine scheme defined by the polynomials

$$
\begin{aligned}
& f_{1}:=\bar{F}_{1}=\beta \gamma \varepsilon u t y-u+\gamma t^{2}, \\
& f_{3}:=\bar{F}_{3}=(\lambda-\delta) t y^{2}+\mu y-\beta \gamma u t^{2}+\beta u^{2}, \\
& f_{4}:=\bar{F}_{4}=(\lambda-\delta) t y+\mu+\beta^{2} \gamma \varepsilon u^{2} t, \\
& f_{5}:=\bar{F}_{5}=\gamma \lambda t^{2} y+\gamma \mu t-\delta u y+\beta^{2} \gamma^{2} \varepsilon u^{2} t^{2}+\beta \gamma \delta \varepsilon u t y^{2}
\end{aligned}
$$

in $\mathbb{A}_{y, t, u}^{3}$. We define

$$
\Delta=\left(f_{1}=f_{3}=f_{4}=f_{5}=0\right) \subset \mathbb{A}_{y, u, t}^{3} .
$$




\section{H. Ahmadinezhad And T. OKada}

We have $f_{3}=y f_{4}-\beta u f_{1}$ and $f_{5}=\gamma t f_{4}+\delta y f_{1}$, which implies that $\Delta$ is defined by $f_{1}=f_{4}=0$. We set

$$
\theta=\frac{\beta \gamma \varepsilon}{\lambda-\delta} \neq 0
$$

and we eliminate the term uty from $f_{1}$; that is, we consider $f_{1}^{\prime}=f_{1}-\theta f_{4}$. Then $\Delta$ is defined by $f_{1}^{\prime}=f_{4}=0$. Here, we have $f_{1}^{\prime}=\theta_{1} u+\gamma t^{2}+\theta_{2} u^{3} t$, where $\theta_{1}=-(\theta \mu+1)$ and $\theta_{2}=-\beta^{2} \gamma \varepsilon \theta$. Note that $\theta_{1}$ can be zero while $\theta_{2} \neq 0$. We have $(t=0) \cap \Delta=\emptyset$ since $\mu \neq 0$. It follows that $\Delta$ is contained in the open subset $(t \neq 0) \subset \mathbb{A}^{3}$. The projection $\mathbb{A}_{y, t, u}^{3} \rightarrow \mathbb{A}_{t, u}^{2}$ induces an isomorphism $\Delta \rightarrow \Xi \cap(t \neq 0)$, where $\Xi$ is the curve in $\mathbb{A}_{y, u}^{2}$ defined by $f_{1}^{\prime}=0$. If $\theta_{1} \neq 0$, it is clear that $\Xi$ is irreducible and reduced, and so is $\Delta$. If $\theta_{1}=0$, then $f_{1}^{\prime}=t\left(\gamma t+\theta_{2} u^{3}\right)$ and $\Xi \cap(t \neq 0)$ is defined by $\gamma t-\theta_{2} u^{3}=0$. Since $\gamma \neq 0$, we know that $\Xi \cap(t \neq 0)$ is irreducible and reduced, and so is $\Delta$. Therefore, $\Delta$ is irreducible and reduced, and so is $\Gamma \cap U$.

We consider $\Gamma \cap\left(z_{0}=0\right)$. Since $\left.F_{2}\right|_{\Pi}=\beta \gamma u t-v z_{0}$, we have $\Gamma \cap\left(z_{0}=0\right)=\Sigma_{1} \cup \Sigma_{2}$, where $\Sigma_{1}=\Gamma \cap\left(z_{0}=t=0\right)$ and $\Sigma_{2}=\Gamma \cap\left(z_{0}=u=0\right) \cap(t \neq 0)$. It is easy to see $\Sigma_{t}=\left\{\mathbf{p}_{y}\right\}$. We have

$$
\begin{aligned}
\Sigma_{2} & =\left(z_{0}=u=\varepsilon y v+\gamma t^{2}=(\lambda-\delta) y^{2}-v=\gamma \lambda t^{2} y+\varepsilon v^{2}+\delta \varepsilon v y^{2}=0\right) \cap(t \neq 0) \\
& =\left(z_{0}=u=\varepsilon y v+\gamma t^{2}=(\lambda-\delta) y^{2}-v=0\right) \cap(t \neq 0),
\end{aligned}
$$

and it is straightforward to see that $\Sigma_{2}$ consists of 2 points. Therefore, $\Gamma$ is an irreducible and reduced curve.

LEMma 6.6. If $\alpha \neq 0$, then $\mathrm{p}$ is not a maximal centre.

Proof. We will show $\tilde{S} \cap \tilde{T}=\tilde{\Gamma}$. We have an isomorphism

$$
E \cong\left(F_{1}^{\mathbf{w}}=F_{2}^{\mathbf{w}}=F_{3}^{\mathbf{w}}=0\right) \subset \mathbb{P}\left(1_{x}, 1_{z_{0}}, 5_{z_{1}}, 2_{t}, 3_{u}, 4_{v}\right) .
$$

Note that $\left.F_{i}^{\mathbf{w}}\right|_{x=z_{1}=0}$ coincides with the lowest-weight part of $\left.\left(\left.F_{i}\right|_{\Pi}\right)\right|_{y=1}$. Hence, we have

$$
F_{1}^{\mathbf{w}}=\varepsilon v-u z_{0}+\gamma t^{2}+f, \quad F_{2}^{\mathbf{w}}=\beta \gamma u t-v z_{0}+g, \quad F_{3}^{\mathbf{w}}=(\lambda-\delta) t+\mu z_{0}^{2}+h,
$$

where $f, g, h \in\left(x, z_{1}\right)$. It is straightforward to see

$$
\tilde{S} \cap \tilde{T} \cap E=\left(x=z_{1}=F_{1}^{\mathbf{w}}=F_{2}^{\mathbf{w}}=F_{3}^{\mathbf{w}}=0\right)
$$

is a finite set of points, which implies $\tilde{S} \cap \tilde{T}=\tilde{\Gamma}$.

Finally, since $\tilde{S} \sim_{\mathbb{Q}} \varphi^{*} A-\frac{1}{4} E=B$ and $\tilde{T} \sim_{\mathbb{Q}} 5 \varphi^{*} A-\frac{5}{4} E=5 B$, we have

$$
(\tilde{T} \cdot \tilde{S} \cdot \tilde{T})=5^{2}\left(A^{3}\right)-\frac{5^{2}}{4^{3}}\left(E^{3}\right)=\frac{5}{4}-\frac{5^{2}}{12}<0 .
$$

Therefore, $\mathrm{p}$ is not a maximal centre by Lemma 3.2 .

Next, we consider the case $\alpha=0$.

Lemma 6.7. If $\alpha=0$, then $\mathrm{p}$ is not a maximal centre.

Proof. We see that $y^{3} x^{2}, y^{2} t$ and $y z_{0}^{2}$ are the only monomials of degree 14 having $\mathbf{w}$-weight $2 / 4$. Note that the coefficients of $t y^{2}$ and $z_{0}^{2} y$ in $F_{3}$ are $\lambda-\delta$ and $\mu$, respectively, and let $\theta$ be the coefficient of $y^{3} x^{2}$ in $F_{3}$. We set $s=\theta y^{2} x^{2}+(\lambda-\delta) t y+\mu z_{0}^{2}$. Since the monomials in $F_{3}$ other than $y^{3} x^{2}, t y^{2}$ and $z_{0}^{2} y$ have $\mathbf{w}$-weight greater than $2 / 4$, we have $\operatorname{ord}_{E}(s) \geqslant 6 / 4$.

We will show that $\left\{x, z_{1}, s\right\}$ isolates $\mathrm{p}$. It is enough to show that

$$
\Sigma:=\left(s=\left.F_{1}\right|_{\Pi}=\cdots=\left.F_{5}\right|_{\Pi}=0\right) \cap \Pi^{\circ} \subset \Pi^{\circ}
$$




\section{Birationally Rigid Pfaffian FAno 3-Folds}

is a finite set of points, where $\Pi^{\circ}=\Pi \cap(y \neq 0)$. For a subset $\Xi$ of $\Pi$ and monomials $g_{1}, \ldots, g_{k}$, we define $\Xi_{g_{1}, \ldots, g_{k}}=\Xi \cap\left(g_{1}=\cdots=g_{k}=0\right)$. We claim $\Sigma^{\circ}:=\Sigma \cap(u \neq 0)=\emptyset$. We have $\Sigma^{\circ}=\Sigma_{t}^{\circ}$ since $\left.F_{2}\right|_{\Pi}=\beta \gamma u t$. Then we see $\Sigma^{\circ}=\emptyset$ since $\left.F_{3}\right|_{\Pi}=\left.s\right|_{\Pi}-v t+\beta u^{2}$ and $u \neq 0$ on $\Sigma^{\circ}$. This implies $\Sigma=\Sigma_{u}$. We have $\left.F_{3}\right|_{\Pi^{\prime}}=\left.s\right|_{\Pi^{\prime}}-v t$, hence $\left.F_{3}\right|_{\Sigma}=-v t$. Thus $\Sigma=\Sigma_{u}=\Sigma_{u, v} \cup \Sigma_{u, t}$. Since $\left.F_{1}\right|_{\Pi_{u}}=\varepsilon y v+\gamma t^{2}$, we have $\Sigma_{u, v} \subset \Sigma_{u, t}$. This shows $\Sigma=\Sigma_{u, t}$, and this set is defined in $\Pi_{u, t}$ by the equations

$$
\mu z_{0}^{2}=\varepsilon y v=\varepsilon v^{2}+\delta \varepsilon v y^{2}=0 .
$$

It is now straightforward to see $\Sigma=\{\mathrm{p}\}$.

Now, since $\operatorname{ord}_{E}\left(x, z_{1}, s\right) \geqslant \frac{1}{4}(1,5,6)$, we see that $L=10 \varphi^{*} A-\frac{6}{4} E$ is nef by Lemma 3.3 and we have

$$
\left(L \cdot B^{2}\right)=10\left(A^{3}\right)-\frac{6}{4^{3}}\left(E^{3}\right)=\frac{1}{2}-\frac{1}{2}=0 .
$$

Therefore, $\mathrm{p}$ is not a maximal centre by Lemma 3.1.

\subsection{The $\frac{1}{5}(1,2,3)$-point and birational involution}

Let $\mathrm{p} \in X$ be the point of type $\frac{1}{5}(1,2,3)$. We may assume $\mathrm{p}=\mathrm{p}_{z_{1}}$ after replacing $z_{0}$ and $z_{1}$. We have $u \in a_{6}, b_{6}$ and $v \in a_{7}, b_{7}$ since $\mathrm{p}_{u}, \mathrm{p}_{v} \notin X$. Since $\mathrm{p}$ is of type $\frac{1}{5}(1,2,3)$, we have $z_{1}^{2} y \in F_{3}$ and $z_{1}^{2} z_{0} \in F_{4}$. Because $z_{1}^{2} y \in F_{3}=a_{4} d_{10}-a_{6} b_{8}+a_{7} b_{7}$, we have $y \in a_{4}$ and $z_{1}^{2} \in d_{10}$. It follows that $z_{1}^{2} t \in F_{5}=b_{6} d_{10}-b_{7} c_{9}+b_{8} c_{8}$. Thus $\varphi$ is the weighted blowup with weight $\operatorname{wt}(x, u, v)=\frac{1}{5}(1,2,3)$. By Lemma 3.10, we can assume that $z_{1}^{2} t$ is the unique monomial in $F_{5}$ divisible by $z^{2}$. We see that $z_{1}^{3} x$ and $z_{1}^{2} t$ are all the monomials of degree 16 having initial weight $\frac{1}{5}$. By our choice of coordinates, $z_{1}^{3} x \notin F_{5}$; hence $\mathrm{wt}\left(x, y, z_{0}, t, u, v\right)=\frac{1}{5}(1,4,5,6,2,3)=: \mathbf{w}$ satisfies the KBL condition.

Let $\pi: X \rightarrow \mathbb{P}:=\mathbb{P}(1,4,5,6)$ be the projection to the coordinates $x, y, z_{0}, t$. We have

$$
F_{3}\left(0,0,0, z_{1}, 0, u, v\right)=\lambda u^{2}, \quad F_{5}\left(0,0,0, z_{1}, 0, u, v\right)=\mu v^{2}
$$

for some $\lambda, \mu \in \mathbb{C} \backslash\{0\}$ since $u \in a_{6}, b_{6}$ and $v \in a_{7} b_{7}$. Hence, we have $\left(x=y=z_{0}=t=0\right) \cap X=$ $\{\mathrm{p}\}$, which implies that $\pi$ is defined outside p. Let $\pi_{Y}: Y \rightarrow \mathbb{P}$ be the induced rational map. We take $H \in\left|\mathcal{O}_{\mathbb{P}}(1)\right|$.

LEMma 6.8. The map $\pi_{Y}$ is a surjective generically finite morphism of degree 2 such that $B=$ $\pi_{Y}^{*} H$.

Proof. First, we show that $\pi_{Y}$ is everywhere defined. It is enough to show that $\pi_{Y}$ is defined at every point of $E$. We see that $\varphi$ is realized as the embedded weighted blowup at $\mathrm{p}$ with weight $\mathbf{w}$, and we have an isomorphism

$$
E \cong\left(F_{3}^{\mathbf{w}}=F_{4}^{\mathbf{w}}=F_{5}^{\mathbf{w}}=0\right) \subset \mathbb{P}\left(1_{x}, 4_{y}, 5_{z_{0}}, 6_{t}, 2_{u}, 3_{v}\right) .
$$

The indeterminacy locus of $\pi_{Y}$ is the set $\left(x=y=z_{0}=t=0\right) \cap E$. We see $F_{3}^{\mathbf{w}}=y+\alpha u^{2}+g_{3}$, $F_{4}^{\mathbf{w}}=z_{0}+\beta v u+g_{4}$ and $F_{5}^{\mathbf{w}}=t+\gamma v^{2}+g_{5}$, where $g_{3}, g_{4}, g_{5} \in\left(x, y, z_{0}, t\right), y \notin g_{3}, z_{0} \notin g_{4}, t \notin g_{5}$ and $\alpha, \beta, \gamma \neq 0$. Hence, the set $\left(x=y=z_{0}=t=0\right) \cap E$ is empty, which shows that $\pi_{Y}$ is a morphism.

By construction, $\pi_{Y}^{*} H$ is the proper transform of $(x=0) \cap X$ via $\varphi$, which is $B$ since $\operatorname{ord}_{E}(x)=\frac{1}{5}$. We have $\left(H^{3}\right)=\frac{1}{120}$ and

$$
\left(B^{3}\right)=\left(A^{3}\right)-\frac{1}{5^{3}}\left(E^{3}\right)=\frac{1}{20}-\frac{1}{30}=\frac{1}{60} .
$$

This implies that $\pi_{Y}$ is a surjective generically finite morphism of degree 2 . 


\section{H. Ahmadinezhad And T. OKada}

Proposition 6.9. One of the following holds:

(i) The point $\mathrm{p}$ is not a maximal centre.

(ii) There is a birational involution $\sigma: X \rightarrow X$ which is a Sarkisov link centred at $\mathrm{p}$.

Proof. We take the Stein factorization of $\pi_{Y}$, let $\psi: Y \rightarrow Z$ be the birational morphism and let $\pi_{Z}: Z \rightarrow \mathbb{P}$ be the double cover such that $\pi_{Y}=\pi_{Z} \circ \psi$. By Lemma 6.10 below, $\psi$ is not an isomorphism. Thus, by [Oka17, Lemma 3.2], either statement (i) or statement (ii) holds, depending on whether $\psi$ is divisorial or small.

We use the following result in the above proof.

Lemma 6.10. Let $X$ be a $\mathbb{Q}$-Fano 3 -fold embedded in a weighted projective space $\mathbb{P}\left(a_{0}, \ldots, a_{n}\right)$. Suppose that $X$ is quasi-smooth, and let $\varphi: Y \rightarrow X$ be the Kawamata blowup of $X$ at a terminal quotient singular point $\mathrm{p} \in X$. Then $Y$ cannot be a double cover of any weighted projective 3space.

Proof. Assume that there is a double cover $\pi: Y \rightarrow \mathbb{P}:=\mathbb{P}\left(b_{0}, \ldots, b_{3}\right)$. Let $D \subset \mathbb{P}$ be the branched divisor and $f$ the defining polynomial of $D$. Then $Y$ is isomorphic to the weighted hypersurface $Z:=\left(y^{2}-f=0\right) \subset \mathbb{P}\left(b_{0}, \ldots, b_{3}, d\right)$, where $2 d=\operatorname{deg} f$ and $d=\operatorname{deg} y$. Since $X$ is quasi-smooth and $\varphi$ is a Kawamata blowup, we see that $Y$ has only (terminal) quotient singularities, and so does $Z \cong Y$. This implies that $Z$ is quasi-smooth, which implies that the Picard number of $Z$ is 1 (see [Dol82, Theorem 3.2.4]). This gives a contradiction since the Picard number of $Y$ is 2 .

\section{Pfaffian Fano 3-fold of degree 1/12}

Let $X=X_{10,11,12,13,14} \subset \mathbb{P}\left(1_{x}, 3_{y}, 4_{z}, 5_{t_{0}}, 5_{t_{1}}, 6_{u}, 7_{v}\right)$ be a Pfaffian Fano 3 -fold of degree $\frac{1}{12}$. The main aim of this section is to prove that there is a Sarkisov link centred at the $\frac{1}{5}(1,2,3)$ point which links to a Mori fibre space other than $X$. This implies that $X$ is not birationally rigid. Unfortunately, we are unable to construct an explicit link. Instead, we will show that the Kawamata blowup at the $\frac{1}{5}(1,2,3)$-point admits a flop (and thus there is a link to a Mori fibre space) and then derive a contradiction assuming that the target of the link is isomorphic to $X$. To do this, we need to exclude or untwist the other centres, so we will exclude singular points of type $\frac{1}{3}(1,1,2)$ and construct a Sarkisov link centred at the $\frac{1}{5}(1,1,4)$-point which is a birational involution. The syzygy matrix of $X$ and the defining polynomials are given as follows:

$$
M=\left(\begin{array}{ccccc}
0 & a_{3} & a_{4} & a_{5} & a_{6} \\
& 0 & b_{5} & b_{6} & b_{7} \\
& & 0 & c_{7} & c_{8} \\
& & & 0 & d_{9} \\
& & & & 0
\end{array}\right), \quad \begin{aligned}
& F_{1}=a_{3} c_{7}-a_{4} b_{6}+a_{5} b_{5}, \\
& F_{2}=a_{3} c_{8}-a_{4} b_{7}+a_{6} b_{5}, \\
& F_{3}=a_{3} d_{9}-a_{5} b_{7}+a_{6} b_{6}, \\
& F_{4}=a_{4} d_{9}-a_{5} c_{8}+a_{6} c_{7}, \\
& F_{5}=b_{5} d_{9}-b_{6} c_{8}+b_{7} c_{7} .
\end{aligned}
$$

The basket of singularities of $X$ is

$$
\left\{2 \times \frac{1}{3}(1,1,2), \frac{1}{4}(1,1,3), \frac{1}{5}(1,1,4), \frac{1}{5}(1,2,3)\right\} .
$$

We have $u \in a_{6}, b_{6}$ and $v \in b_{7}, c_{7}$ since $\mathrm{p}_{u}, \mathrm{p}_{v} \notin X$. 


\section{Birationally Rigid Pfaffian FAno 3-Folds}

\subsection{Exclusion of the $\frac{1}{4}(1,1,3)$-point}

Let $\mathrm{p}=\mathrm{p}_{z}$ be the point of type $\frac{1}{4}(1,1,3)$. For the entries $a_{5}, b_{5}, d_{9}$ of the syzygy matrix $M$, we write $a_{5}=\ell_{1}+$ (other terms), $b_{5}=\ell_{2}+$ (other terms) and $d_{9}=z \ell_{3}+$ (other terms), where $\ell_{i}=\ell_{i}\left(t_{0}, t_{1}\right)$ is a linear form. We see that the solutions of $x=y=z=u=v=\ell_{1} \ell_{2}=0$ correspond to the types $\frac{1}{5}(1,1,4)$ and $\frac{1}{5}(1,2,3)$, so that $\ell_{1} \neq 0$ and $\ell_{2}$ are not proportional. We assume $z \in a_{4}$. Then we can assume that the coefficient of $z$ in $a_{4}$ is 1 by re-scaling $z$ and let $\varepsilon \in \mathbb{C}$ be the coefficient of $z^{2}$ in $c_{8}$.

Lemma 7.1. We have $\ell_{3} \neq 0$, and $\ell_{1}$ and $\ell_{3}$ are not proportional.

Proof. We have

$$
F_{1}=\ell_{1} \ell_{2}+\cdots, \quad F_{3}=-\ell_{1} v+\cdots, \quad F_{4}=z^{2} \ell_{3}-\delta z^{2} \ell_{1} \cdots, \quad F_{5}=z \ell_{2} \ell_{3}+\cdots .
$$

Let $\mathrm{q}_{1}$ and $\mathrm{q}_{2}$ be the singular points corresponding to the solutions $\ell_{1}=0$ and $\ell_{2}=0$, respectively. We see that $\mathrm{q}_{2}$ is of type $\frac{1}{5}(1,1,4)$ since $F_{3}=\ell_{1} v+\cdots$; hence $\mathrm{q}_{1}$ is of type $\frac{1}{5}(1,2,3)$. Assume $\ell_{3}=\theta \ell_{1}$ for some $\theta \in \mathbb{C}$. Then $F_{3}=\theta z \ell_{1} \ell_{2}+\cdots$, and this implies $(\partial F / \partial z)\left(\mathbf{q}_{2}\right)=0$. This gives a contradiction since $\mathrm{q}_{2}$ is of type $\frac{1}{5}(1,1,4)$, and the proof is completed.

We exclude the point $\mathrm{p}$ assuming the following.

Condition 7.2. We have $z \in a_{4}$ and, under the above choice of coordinates, $\ell_{3}-\delta \ell_{1} \nsim \ell_{2}$.

We have $u^{2} \in F_{3}$ and $t^{2} \in F_{5}$ since $\mathrm{p}_{u}, \mathrm{p}_{v} \notin X$, which implies $u \in a_{6}, b_{6}$ and $v \in b_{7}, c_{7}$. We have $F_{4}=z^{2}\left(\ell_{3}-\varepsilon \ell_{1}\right)+\cdots$ and $\ell_{3}-\varepsilon \ell_{1} \neq 0$ by Lemma 7.1. Replacing $t_{0}$ and $t_{1}$, we may assume $\ell_{3}-\delta \ell_{1}=t_{0}$. By Lemma 3.10, after further replacing $t_{0}$, we can assume that $z^{2} t_{0}$ is the unique monomial in $F_{4}$ which is divisible by $z^{2}$. Set $\Pi=\left(x=y=t_{0}=0\right)$. Then the restriction of $M$ and the defining polynomials to $\Pi$ can be written as follows:

$$
\left.M\right|_{\Pi}=\left(\begin{array}{ccccc}
0 & 0 & z & \alpha t_{1} & u \\
& 0 & \beta t_{1} & \gamma u & v \\
& 0 & \delta v & \varepsilon z^{2} \\
& & 0 & \zeta z t_{1} \\
& & & 0
\end{array}\right), \quad \begin{aligned}
& \left.F_{1}\right|_{\Pi}=-\gamma z u+\alpha \beta t_{1}^{2}, \\
& \left.F_{2}\right|_{\Pi}=-z v+\beta u t_{1}, \\
& \left.F_{3}\right|_{\Pi}=-\alpha t_{1} v+\gamma u^{2}, \\
& \left.F_{4}\right|_{\Pi}=(\zeta-\alpha \varepsilon) z^{2} t_{1}+\delta u v \\
& \left.F_{5}\right|_{\Pi}=\beta \zeta z t_{1}^{2}-\gamma \varepsilon u z^{2}+\delta v^{2}
\end{aligned}
$$

for some $\alpha, \beta, \ldots, \zeta \in \mathbb{C}$ with $\gamma, \delta \neq 0$. By our choice of coordinates, we have $z^{2} t_{1} \notin F_{4}$, that is, $\zeta-\alpha \varepsilon=0$.

LEMMA 7.3. The point $\mathrm{p}$ of type $\frac{1}{4}(1,1,3)$ is not a maximal centre.

Proof. We see $z u \in F_{1}, z v \in F_{2}$ and $z^{2} t_{0} \in F_{4}$. We see that $z^{3} x$ and $z^{2} t_{0}$ are the only monomials of degree 13 having initial weight $\frac{1}{4}$. By our choice of coordinates, we have $z^{3} x \notin F_{4}$. This implies that the weight $\operatorname{wt}\left(x, y, t_{0}, t_{1}, u, v\right)=\frac{1}{4}(1,3,5,1,2,3)$ satisfies the KBL condition.

We claim that none of $\alpha$ and $\beta$ is zero. If $\alpha=0$, then $\ell_{1} \sim t_{0}$. Since $\ell_{3}-\varepsilon \ell_{1}=z_{0}$, this implies $\ell_{3} \sim \ell_{1}$. This is impossible. If $\beta=0$, then $\ell_{2} \sim t_{0}$, and this is impossible by Condition 7.2.

It is now straightforward to check $X \cap \Pi=\{\mathrm{p}\}$ since $\alpha, \beta, \gamma, \delta \neq 0$. In particular, $\left\{x, y, t_{0}\right\}$ isolates $\mathrm{p}$. We have $\operatorname{ord}_{E}\left(x, y, t_{0}\right) \geqslant \frac{1}{4}(1,3,5)$, so that $L=B$ is nef by Lemma 3.3 , and we compute

$$
\left(L \cdot B^{2}\right)=\left(A^{3}\right)-\frac{1}{4^{3}}\left(E^{3}\right)=\frac{1}{12}-\frac{1}{12}=0 .
$$

Therefore, $\mathrm{p}$ is not a maximal centre by Lemma 3.1. 


\section{H. Ahmadinezhad And T. OKada}

\subsection{The $\frac{1}{3}(1,1,2)$-point}

Let $\mathrm{p}$ be a point of type $\frac{1}{3}(1,1,2)$. After replacing coordinates, we may assume $\mathrm{p}=\mathrm{p}_{y}$. We assume $y \in a_{3}$. Then, re-scaling $y$, we can assume that the coefficient of $y$ in $a_{3}$ is 1 . We see $y v \in F_{1}$ and, replacing $v$, we may assume that $y v$ is the unique monomial in $F_{1}$ divisible by $y$. We can write the entries of the syzygy matrix as $a_{5}=\ell_{1}+$ (other terms), $b_{5}=\ell_{2}+$ (other terms), $c_{8}=y \ell_{3}+\eta z^{2}+\left(\right.$ other terms) and $d_{9}=z \ell_{4}+$ (other terms) for some linear forms $\ell_{1}, \ldots, \ell_{4}$ in $t_{0}$ and $t_{1}$ and $\eta \in \mathbb{C}$. Let $\alpha, \beta$ and $\delta$ be the coefficients of $z, y^{2}$ and $z y$ in $a_{4}, a_{6}$ and $b_{7}$, respectively.

Lemma 7.4. We have $\ell_{1}, \ell_{2} \neq 0$. Moreover, $\ell_{1} \nsim \ell_{2}, \ell_{1} \nsim \ell_{4}$ and $\ell_{2} \nsim \ell_{3}$.

Proof. The set

$$
(x=y=z=u=v=0) \cap X=\left(x=y=z=u=v=\ell_{1} \ell_{2}=0\right)
$$

consists of 2 singular points of type $\frac{1}{5}(1,2,3)$ and $\frac{1}{5}(1,1,4)$, respectively, which implies $\ell_{1} \ell_{2} \neq 0$ and $\ell_{1} \nsim \ell_{2}$. In this proof, we assume $\ell_{1}=t_{0}$ and $\ell_{2}=t_{1}$ after replacing $t_{0}$ and $t_{1}$. Since $F_{3}=a_{3} d_{9}-a_{5} b_{7}+a_{6} b_{6}, v \in b_{7}$ and $v \notin a_{3}, d_{9}, a_{5}, a_{6}, b_{6}$, we see $v t_{0} \in F_{1}$ and $v t_{1} \notin F_{1}$. This shows that $\mathrm{p}_{t_{0}}$ and $\mathrm{p}_{t_{1}}$ are of type $\frac{1}{5}(1,1,4)$ and $\frac{1}{5}(1,2,3)$, respectively.

Assume $\ell_{3} \sim \ell_{2}$, that is, $\ell_{3}=\nu t_{1}$ for some $\nu \in \mathbb{C}$. Since $\mathrm{p}_{t_{0}}$ is of type $\frac{1}{5}(1,1,4)$, we have $t_{0}^{2} y \in F_{4}$. But since $\left.F_{4}\right|_{\Pi}=-\ell_{1} \ell_{3}+\cdots, \ell_{1}=t_{0}$ and $\ell_{3}=\nu t_{1}$, we see $t_{0}^{2} y \notin F_{4}$. This gives a contradiction.

Assume $\ell_{4} \sim \ell_{1}$, that is, $\ell_{4}=\nu t_{0}$ for some $\nu \in \mathbb{C}$. Since $\mathrm{p}_{t_{1}}$ is of type $\frac{1}{5}(1,2,3)$, we have $t_{1}^{2} z \in F_{5}$. But since $\left.F_{5}\right|_{\Pi}=z \ell_{2} \ell_{4}+\cdots, \ell_{2}=t_{1}$ and $\ell_{4}=\nu t_{0}$, we see $t_{1}^{2} \notin F_{5}$. This gives a contradiction and the proof is completed.

We exclude the point $\mathrm{p}$ assuming the following generality condition.

Condition 7.5. We have $y \in a_{3}, \eta-\alpha \delta \neq 0, \ell_{3}+\beta \ell_{2} \nsim \ell_{1}$ and $\ell_{4}-\delta \ell_{1} \nsim \ell_{2}$.

Note that $u \in a_{6}, b_{6}$ and $v \in b_{7}, c_{7}$ since $\mathrm{p}_{u}, \mathrm{p}_{v} \notin X$. We set $\Pi=(x=u=v=0)$. Then we can write

$$
\left.M\right|_{\Pi}=\left(\begin{array}{ccccc}
0 & y & \alpha z & \ell_{1} & \beta y^{2} \\
& 0 & \ell_{2} & \gamma y^{2} & \delta z y \\
& & 0 & \varepsilon z y & y \ell_{3}+\eta z^{2} \\
& & & 0 & z \ell_{4}+\zeta y^{3} \\
& & & & 0
\end{array}\right) .
$$

We see that the coefficients of $z y^{2}$ and $y^{4}$ in $\left.F_{1}\right|_{\Pi}$ and $\left.F_{3}\right|_{\Pi}$ are $\varepsilon-\alpha \gamma$ and $\zeta+\beta \gamma$, respectively, and both of them are zero by our choice of coordinates. By eliminating $\varepsilon=\alpha \gamma$ and $\zeta=-\beta \gamma$, we have

$$
\begin{aligned}
& \left.F_{1}\right|_{\Pi}=\ell_{1} \ell_{2}, \\
& \left.F_{2}\right|_{\Pi}=y^{2}\left(\ell_{3}+\beta \ell_{2}\right)+(\eta-\alpha \delta) z^{2} y, \\
& \left.F_{3}\right|_{\Pi}=\left(\ell_{4}-\delta \ell_{1}\right) z y, \\
& \left.F_{4}\right|_{\Pi}=-y \ell_{1} \ell_{3}+z^{2}\left(\alpha \ell_{4}-\eta \ell_{1}\right), \\
& \left.F_{5}\right|_{\Pi}=-\gamma\left(\ell_{3}+\beta \ell_{2}\right) y^{3}-\gamma(\eta-\alpha \delta) z^{2} y^{2}+z \ell_{2} \ell_{4} .
\end{aligned}
$$

Lemma 7.6. No singular point of type $\frac{1}{3}(1,1,2)$ is a maximal centre.

Proof. We will show that $\{x, u, v\}$ isolates p. It is enough to show that $X \cap \Pi^{\circ}$ is a finite set of points, where $\Pi^{\circ}=\Pi \cap(y \neq 0)$. We have $\left.F_{5}\right|_{\Pi}+\left.\gamma y F_{2}\right|_{\Pi}=z \ell_{2} \ell_{4}$. Since $\left.F_{1}\right|_{\Pi}=\ell_{1} \ell_{2}$,

$$
X \cap \Pi^{\circ}=\left(\ell_{1} \ell_{2}=\left.F_{2}\right|_{\Pi}=\left.F_{3}\right|_{\Pi}=\left.F_{4}\right|_{\Pi}=z \ell_{2} \ell_{4}=0\right) \cap \Pi^{\circ}=\Sigma_{1} \cup \Sigma_{2},
$$




\section{Birationally Rigid Pfaffian FAno 3-FOLdS}

where

$$
\begin{aligned}
& \Sigma_{1}=\left(\ell_{1}=\left.F_{2}\right|_{\Pi}=\left.F_{3}\right|_{\Pi}=\left.F_{4}\right|_{\Pi}=z \ell_{2} \ell_{4}=0\right) \cap \Pi^{\circ}, \\
& \Sigma_{2}=\left(\ell_{2}=\left.F_{2}\right|_{\Pi}=\left.F_{3}\right|_{\Pi}=\left.F_{4}\right|_{\Pi}=0\right) \cap \Pi^{\circ} .
\end{aligned}
$$

Since $\ell_{1} \nsim \ell_{2}$ and $\ell_{1} \nsim \ell_{4}$, the equalities $\ell_{1}=\ell_{2}=0$ and $\ell_{1}=\ell_{4}=0$ both imply $t_{0}=t_{1}=0$. Hence, we have $\left(\ell_{1}=z \ell_{2} \ell_{4}=0\right)=\left(t_{0}=t_{1}=0\right) \cup\left(\ell_{1}=z=0\right)$ and

$$
\Sigma_{1}=\left(\left(t_{0}=t_{1}=(\eta-\alpha \delta) z^{2} y=0\right) \cap \Pi^{\circ}\right) \cup\left(\left(\ell_{1}=z=\ell_{3}+\beta \ell_{2}=0\right) \cap \Pi^{\circ}\right)=\{\mathrm{p}\},
$$

since $\eta-\alpha \delta \neq 0$ and $\ell_{3}+\beta \ell_{2} \nsim \ell_{1}$ by Condition 7.5.

Since $\left.F_{3}\right|_{\Pi}=\left(\ell_{4}-\delta \ell_{1}\right) z y$ and $\ell_{4}-\delta \ell_{1} \nsim \ell_{2}$ by Condition 7.5, we have $\left(\ell_{2}=\left.F_{3}\right|_{\Pi}=0\right) \cap \Pi^{\circ}=$ $\left(t_{0}=t_{1}=0\right) \cup\left(\ell_{2}=z=0\right)$. Hence

$$
\Sigma_{2}=\left(\left(t_{0}=t_{1}=(\eta-\alpha \delta) z^{2} y=0\right) \cap \Pi^{\circ}\right) \cup\left(\left(\ell_{2}=z=y^{2} \ell_{3}=-y \ell_{1} \ell_{3}=0\right) \cap \Pi^{\circ}\right)=\{\mathrm{p}\},
$$

since $\ell_{3} \nsim \ell_{2}$. Thus, $\{x, u, v\}$ isolates $\mathrm{p}$.

We see that $y^{3} x, y^{2} z, y v$ are the monomials of degree 10 having initial weight $\frac{1}{4}$, and we have $y^{3} x, y^{2} z \notin F_{1}$ by our choice of coordinates. Hence, we have $\operatorname{ord}_{E}(x, u, v) \geqslant \frac{1}{3}(1,3,4)$ and $L=6 \varphi^{*} A-\frac{3}{3} E$ is nef by Lemma 3.3. We compute

$$
\left(L \cdot B^{2}\right)=6\left(A^{3}\right)-\frac{3}{3^{3}}\left(E^{3}\right)=\frac{1}{2}-\frac{1}{2}=0 .
$$

Therefore $\mathrm{p}$ is not a maximal centre by Lemma 3.1 .

\subsection{The $\frac{1}{5}(1,1,4)$-point and birational involution}

Let $\mathrm{p} \in X$ be the point of type $\frac{1}{5}(1,1,4)$. We may assume $\mathrm{p}=\mathrm{p}_{t_{1}}$ after replacing $t_{0}$ and $t_{1}$. Then we have $t_{1} t_{0} \in F_{1}, t_{1} v \in F_{3}$ and $t_{1}^{2} y \in F_{4}$ since $\mathrm{p}$ is of type $\frac{1}{5}(1,1,4)$. We have $u \in a_{6}, b_{6}$ and $v \in b_{7}, c_{7}$ since $\mathrm{p}_{u}, \mathrm{p}_{v} \notin X$. We see that $\varphi$ is the weighted blowup of $X$ at $\mathrm{p}$ with weight $\operatorname{wt}(x, z, u)=\frac{1}{5}(1,4,1)$, and it is realized as the embedded weighted blowup with the initial weight $\operatorname{wt}\left(x, y, z, t_{0}, u, v\right)=\mathbf{w}_{\text {in }}=\frac{1}{5}(1,3,4,5,1,2)$.

Let $\pi: X \rightarrow \mathbb{P}:=\mathbb{P}(1,3,4,5)$ be the projection to the coordinates $x, u, z, t_{0}$, and let $\pi_{Y}: Y \rightarrow \mathbb{P}$ the induced rational map. We take $H \in\left|\mathcal{O}_{\mathbb{P}}(1)\right|$.

Lemma 7.7. The map $\pi_{Y}$ is a surjective generically finite morphism of degree 2 such that $B=$ $\pi_{Y}^{*} H$.

Proof. We will show that $\pi_{Y}$ is everywhere defined. We have an isomorphism

$$
E \cong\left(F_{1}^{\mathbf{w}_{\text {in }}}=F_{3}^{\mathbf{w}_{\text {in }}}=F_{4}^{\mathbf{w}_{\text {in }}}=0\right) \subset \mathbb{P}\left(1_{x}, 3_{y}, 4_{z}, 5_{t_{0}}, 1_{u}, 2_{v}\right),
$$

and it is enough to show $\left(x=y=z=t_{0}=0\right) \cap E=\emptyset$. We can write $F_{1}^{\mathbf{w}_{\text {in }}}=t_{0}+g_{1}$, $F_{3}^{\mathbf{w}_{\text {in }}}=v+\alpha u^{2}+g_{3}$ and $F_{4}^{\mathbf{w}_{\text {in }}}=y+\beta v u+g_{4}$, where $g_{i} \in\left(x, y, z, t_{0}\right)$ and $\alpha, \beta \in \mathbb{C} \backslash\{0\}$. It is now clear that $\left(x=y=z=t_{0}=0\right) \cap E=\emptyset$. This shows that $\pi_{Y}$ is a morphism. We have $B=\pi_{Y}^{*} H$ since the section $x$ lifts to an anticanonical section on $Y$. We have $\left(H^{3}\right)=1 / 60$ and

$$
\left(B^{3}\right)=\left(A^{3}\right)-\frac{1}{5^{3}}\left(E^{3}\right)=\frac{1}{12}-\frac{1}{20}=\frac{1}{30},
$$

which shows that $\pi_{Y}$ is surjective and is generically finite of degree 2 .

By the same argument as in the proof of Proposition 6.9, this lemma implies the following. 


\section{H. Ahmadinezhad And T. OKada}

Proposition 7.8. One of the following holds:

(i) The point $\mathrm{p}$ is not a maximal centre.

(ii) There is a birational involution $\sigma: X \rightarrow X$ which is a Sarkisov link centred at $\mathrm{p}$.

\subsection{The $\frac{1}{5}(1,2,3)$-point and birational non-rigidity}

Let $\mathrm{p}$ be the point of type $\frac{1}{5}(1,2,3)$. We will show that there is a Sarkisov link to a Mori fibre space which is not isomorphic to $X$, starting with the Kawamata blowup $\varphi$. We denote by q $\in X$ the unique singular point of type $\frac{1}{5}(1,1,4)$.

Lemma 7.9. By a choice of coordinates, we can assume $\mathrm{p}=\mathrm{p}_{t_{1}}, \mathrm{q}=\mathrm{p}_{t_{0}}$ and that the defining polynomials of $X$ are of the form

$$
\begin{aligned}
& F_{1}=t_{1} t_{0}+v a_{3}+u a_{4}+f_{10}, \\
& F_{2}=t_{1} u+v b_{4}+u b_{5}+g_{11}, \\
& F_{3}=t_{0} v+v c_{5}+\alpha u^{2}+u h_{6}+h_{12}, \\
& F_{4}=t_{0}^{2} y+t_{0}\left(v d_{1}+u d_{2}+h_{8}\right)-\beta u v+v h_{6}^{\prime}+u h_{7}+h_{12}, \\
& F_{5}=t_{1}^{2} z+t_{1}\left(v e_{2}+u e_{3}+g_{9}\right)+\beta v^{2}+v u e_{1}+v g_{7}+u^{2} e_{2}+u g_{8}+g_{14}
\end{aligned}
$$

for some $\alpha, \beta \in \mathbb{C} \backslash\{0\}, a_{i}, b_{i}, \ldots, f_{i} \in \mathbb{C}[x, y, z], g_{i} \in \mathbb{C}\left[x, y, z, t_{0}\right]$ and $h_{i}, h_{6}^{\prime} \in \mathbb{C}\left[x, y, z, t_{1}\right]$ with $t_{1}^{2} y \notin h_{12}$ and $t_{0}^{2} z \notin g_{14}$. Moreover, if $X$ is general, then Condition 7.11 below is satisfied.

Proof. The syzygy matrix can be written as

$$
M=\left(\begin{array}{cccc}
a_{3} & a_{4} & A_{5} & A_{6} \\
0 & B_{5} & \alpha u+t_{0} b_{1}+t_{1} b_{1}^{\prime}+b_{6} & B_{7} \\
0 & 0 & -\beta v+u c_{1}+t_{0} c_{2}+t_{1} c_{2}^{\prime}+c_{7} & v c_{1}^{\prime}+u c_{2}^{\prime \prime}+t_{0} c_{3}+t_{1} c_{3}^{\prime}+c_{8} \\
0 & 0 & 0 & v d_{2}+u d_{3}+t_{0} d_{4}+t_{1} d_{4}^{\prime}+d_{9}
\end{array}\right),
$$

where $\alpha, \beta \in \mathbb{C}, a_{i}, b_{i}, b_{i}^{\prime}, c_{i}, c_{i}^{\prime}, c_{i}^{\prime \prime}, d_{i}, d_{i}^{\prime} \in \mathbb{C}[x, y, z]$ and $A_{i}, B_{i} \in \mathbb{C}\left[x, y, z, t_{0}, t_{1}, u, v\right]$. We will choose suitable coordinates such that the defining polynomials of $X$ are in the desired forms. First, we choose $t_{0}$ and $t_{1}$ such that

$$
A_{5}=t_{0}+a_{4} b_{1}^{\prime}-a_{3} c_{2}^{\prime}, \quad B_{5}=t_{1}+a_{4} b_{1}-a_{3} c_{2} .
$$

Then $t_{1} t_{0}$ is the unique monomial in $F_{1}$ that involves only on $t_{0}$ and $t_{1}$, so that $\mathrm{p}_{t_{0}}$ and $\mathrm{p}_{t_{1}}$ are the $\frac{1}{5}(1,1,4)$ - and $\frac{1}{5}(1,2,3)$-points. We are going to arrange the coordinates such that $\mathrm{p}_{t_{0}}$ and $\mathrm{p}_{t_{1}}$ are of type $\frac{1}{5}(1,1,4)$ and $\frac{1}{5}(1,2,3)$, respectively. Since $\mathrm{p}_{u}, \mathrm{p}_{v} \notin X$, we have $u \in A_{6}, v \in B_{7}$ and $\alpha, \beta \neq 0$. It follows that we can choose $u$ and $v$ such that

$$
A_{6}=u-a_{3} c_{3}^{\prime}, \quad B_{7}=-v+u b_{1}+a_{3}\left(d_{4}-b_{1} c_{3}^{\prime}\right) .
$$

By the quasi-smoothness of $X$ at $\mathrm{p}_{t_{0}}$ and $\mathrm{p}_{t_{1}}$, we have $t_{0}^{2} y \in F_{4}$ and $t_{0}^{2} z \in F_{5}$, which imply $y \in c_{3}$ and $z \in d_{4}^{\prime}$, respectively. Hence, we can choose $y$ and $z$ such that $c_{3}=-y$ and $d_{4}^{\prime}=z+b_{1}^{\prime} c_{3}^{\prime}$. Under the above choice of coordinates, the polynomials $F_{1}, \ldots, F_{5}$ are in the desired forms.

We have

$$
\begin{aligned}
& F_{1}=t_{1} t_{0}+v\left(-\beta a_{3}\right)+(\text { other terms }), \\
& F_{2}=t_{1} u+v\left(a_{3} c_{1}^{\prime}+a_{4}\right)+(\text { other terms }), \\
& F_{5}=t_{1}^{2} z+t_{1} v\left(d_{2}-b_{1}^{\prime} c_{1}^{\prime}-c_{2}^{\prime}\right)+\beta v^{2}+(\text { other terms }) .
\end{aligned}
$$




\section{Birationally Rigid Pfaffian FAno 3-Folds}

Clearly, $y \in-\beta a_{3}$ and $z \in a_{3} c_{1}^{\prime}+a_{4}$ for a general $X$ since $\beta \neq 0$. We see that the set

$$
\left(-\beta a_{3}=a_{3} c_{1}^{\prime}+a_{4}=z+v\left(d_{2}-b_{1}^{\prime} c_{1}^{\prime}-c_{2}^{\prime}\right)+\beta v^{2}=0\right)
$$

consists of 2 distinct points for a general $X$, and the proof is completed.

Remark 7.10. Under the above choice of coordinates, $\mathrm{p}_{t_{1}}$ is of type $\frac{1}{5}\left(1_{x}, 2_{v}, 3_{y}\right)$ and $\mathrm{p}_{t_{0}}$ is of type $\frac{1}{5}\left(1_{x}, 1_{u}, 4_{z}\right)$.

We assume the following condition, which is satisfied for a general $X$ by the above lemma.

Condition 7.11. We have $y \in a_{3}$ and, under the above choice of coordinates, the set

$$
\left(a_{3}=b_{4}=z+v e_{2}+\beta v^{2}=0\right) \subset \mathbb{P}\left(1_{x}, 3_{y}, 4_{z}, 2_{v}\right)
$$

consists of 2 distinct points.

We see that each monomial in $F_{2}=t_{1} u+v b_{4}+u b_{5}+g_{11}$ has initial weight at least $6 / 5$ except for $t_{1} u$, so that the weight $\mathrm{wt}\left(x, y, z, t_{0}, u, v\right)=\frac{1}{5}(1,3,4,5,6,2)=$ : $\mathbf{w}$ satisfies the KBL condition. It follows that $\varphi$ is realized as the embedded weighted blowup with weight $\mathbf{w}$, and we have an isomorphism

$$
E \cong\left(t_{0}+v a_{3}=u+v b_{4}=z+v e_{2}+\beta v^{2}=0\right) \subset \mathbb{P},
$$

where $\mathbb{P}=\mathbb{P}\left(1_{x}, 3_{y}, 4_{z}, 5_{t_{0}}, 6_{u}, 2_{v}\right)$. Let $X \rightarrow \mathbb{P}(1,3,4,5,6)$ be the projection to $x, y, z, t_{0}, u$ which is defined outside $\mathrm{p}$, and denote by $Z$ its image. Let $\rho: Y \rightarrow Z$ be the induced birational map.

Lemma 7.12. The map $\rho$ is a birational morphism, and it is the anticanonical model of $Y$.

Proof. We see that the sections $x, y, z, t_{0}, u$ lift to plurianticanonical sections on $Y$ and that they restrict to $E$ the coordinates $x, y, z, t, u$ of $\mathbb{P}$. It is straightforward to see

$$
(x=y=z=t=u=0) \cap E=\emptyset,
$$

which implies that $\rho$ is everywhere defined. For a general point of $Z$, its inverse image via $\rho$ is a single point since we can solve $t_{1}$ and $v$ in terms of $F_{1}=F_{2}=0$, which can be expressed as

$$
\left(\begin{array}{cc}
t_{0} & a_{3} \\
u & b_{4}
\end{array}\right)\left(\begin{array}{c}
t_{1} \\
v
\end{array}\right)=-\left(\begin{array}{l}
u a_{4}+f_{10} \\
u b_{5}+g_{11}
\end{array}\right) \text {. }
$$

This shows that $\rho$ is birational and thus it is the anticanonical model of $Y$.

The following lemma will be used in order to show that $\rho$ is a small contraction.

Lemma 7.13. Let $V$ be a $\mathbb{Q}$-Fano variety of Picard number 1 , and let $\varphi: W \rightarrow V$ be a $K_{W^{-}}$ negative extremal divisorial contraction with exceptional divisor E. Suppose that $W$ admits a $K_{W}$-trivial divisorial contraction $\psi: W \rightarrow U$ which contracts a divisor $G$. If a prime divisor $D$ on $W$ is $\mathbb{Q}$-linearly equivalent to $-\lambda K_{W}-\mu E$ for some $\lambda$, $\mu$ with $\mu>0$, then $D=G$.

Proof. Note that $\operatorname{Pic}(V) \otimes \mathbb{Q}$ is generated by $-K_{W}$ and $E$, and the cone of effective divisors on $W$ is generated by $E$ and $G$.

Since $\psi: W \rightarrow U$ is divisorial and $-K_{W}$-trivial, there are infinitely many curves on $W$ contracted by $\psi$ and they intersect $-K_{W}$ trivially and $E$ positively. By [Oka17, Lemma 2.20] (see also [CP17]), the contraction $\varphi: W \rightarrow V$ is not a maximal extraction. This implies that a divisor which is $\mathbb{Q}$-linearly equivalent to $-\lambda^{\prime} K_{W}-\mu^{\prime} E$ is not mobile if $\mu^{\prime}>0$ (because otherwise $\varphi$ is a maximal extraction). 


\section{H. Ahmadinezhad And T. OKada}

Let $D \sim_{\mathbb{Q}}-\lambda K_{W}-\mu E$, where $\mu>0$, be a prime divisor. We assume $D \neq G$. Since the cone of effective divisor of $W$ is generated by $E$ and $G$, we can write $D \sim_{\mathbb{Q}} k G+l E$ for some rational numbers $k, l>0$. Take a positive integer $m$ such that $m D \sim m k G+m l E$ and $m k, m l \in \mathbb{Z}$. This linear equivalence implies that the linear system $|m D|$ is mobile since $D \neq G, E$. This gives a contradiction and the assertion is proved.

LEMma 7.14. The map $\rho$ is a flopping contraction.

Proof. We see that the set

$$
\left(a_{4}=b_{4}=0\right) \cap E=\left(a_{3}=b_{4}=t_{0}=u=z+v e_{2}+v^{2}=0\right) \subset \mathbb{P}
$$

consists of 2 points $\left\{\mathbf{q}_{1}, \mathbf{q}_{2}\right\}$ and that both of them are mapped to the same point $\mathbf{q} \in \rho(E)$ via $\rho$, where

$$
\{\mathrm{q}\}=\left(a_{3}=b_{4}=t_{0}=u=0\right) \subset \mathbb{P}(1,3,4,5,6) .
$$

Note that this in particular implies that $\rho$ is not an isomorphism.

It remains to show that $\rho$ is not divisorial. Assume that $\rho$ is divisorial, and let $G$ be the prime divisor on $Y$ contracted by $\rho$. Since $G$ is contracted by the $B$-trivial contraction $\rho$, we have $\left(B^{2} \cdot G\right)=0$. Since $\left(B^{3}\right)=1 / 20$, we compute

$$
0=\left(B^{2} \cdot G\right)=k\left(B^{3}\right)-l\left(B^{2} \cdot E\right)=\frac{1}{20} k-\frac{1}{5^{2}} l\left(E^{3}\right)=\frac{1}{20} k-\frac{1}{6} l .
$$

Since $k$ and $l$ are integers, we have $G \sim_{\mathbb{Q}} m(10 B-3 E)$ for some positive integer $m$. We will construct a prime divisor on $Y$ which is $\mathbb{Q}$-linearly equivalent to $\lambda B-\mu E$ for some $\lambda$ and $\mu$ with $0<\lambda<10$ and $\mu>0$. We have

$$
b_{4} F_{1}-a_{3} F_{2}=t_{1}\left(t_{0} b_{4}-u a_{3}\right)+b_{4}\left(u a_{4}+f_{10}\right)-a_{3}\left(u b_{5}+g_{11}\right) .
$$

Thus, on $X$, we have

$$
t_{1}\left(t_{0} b_{4}-u a_{3}\right)=-b_{4}\left(u a_{4}+f_{10}\right)+a_{3}\left(u b_{5}+g_{11}\right) .
$$

Each monomial on the right-hand side of this equation vanishes along $E$ to order at least 14/5. Let $H \sim_{\mathbb{Q}} 9 A$ be the divisor on $X$ defined by $t_{0} b_{4}-u a_{3}=0$. We have $\tilde{H} \sim_{\mathbb{Q}} 9 \varphi^{*} A-\frac{14}{5} E=9 B-E$. Note that $\tilde{H}$ is not necessarily irreducible or reduced. However, there is a prime divisor $D$ (which is a component of $\tilde{H}$ ) such that $D \sim_{\mathbb{Q}} \lambda B-\mu E$ with $\mu>0$. The integer $\lambda$ necessarily satisfies $0<\lambda \leqslant 9$. This implies $D \neq G$. By Lemma 7.13, this gives a contradiction and $\rho$ is small.

Let $\varphi^{\prime}: Y^{\prime} \rightarrow X$ be the Kawamata blowup of $X$ at the $\frac{1}{5}(1,1,4)$-point $\mathrm{q}=\mathrm{p}_{t_{0}}$ with exceptional divisor $E^{\prime}$. We see that $\varphi^{\prime}$ can be realized as the embedded weighted blowup with the initial weight $\operatorname{wt}\left(x, y, z, t_{1}, u, v\right)=\frac{1}{5}(1,3,4,5,1,2)$, so that we have an isomorphism

$$
E^{\prime} \cong\left(t_{1}+v a_{3}+u a_{4}=v+\alpha u^{2}=y+v d_{1}+u d_{2}-\beta u v=0\right) \subset \mathbb{P}^{\prime},
$$

where $\mathbb{P}^{\prime}=\mathbb{P}\left(1_{x}, 3_{y}, 4_{z}, 5_{t_{1}}, 1_{u}, 2_{v}\right)$.

Let $\psi: \hat{Y} \rightarrow Y$ be the Kawamata blowup of $Y$ at the $\frac{1}{5}(1,1,4)$-point $\varphi^{-1}(\mathrm{q})$. We denote by $\pi: X \rightarrow \mathbb{P}(1,3,4)$ the projection to $x, y, z$ and by $\eta: \hat{Y} \rightarrow \mathbb{P}(1,3,4)$ the induced rational map. 


\section{Birationally Rigid Pfaffian FAno 3-Folds}

We have the following diagram:

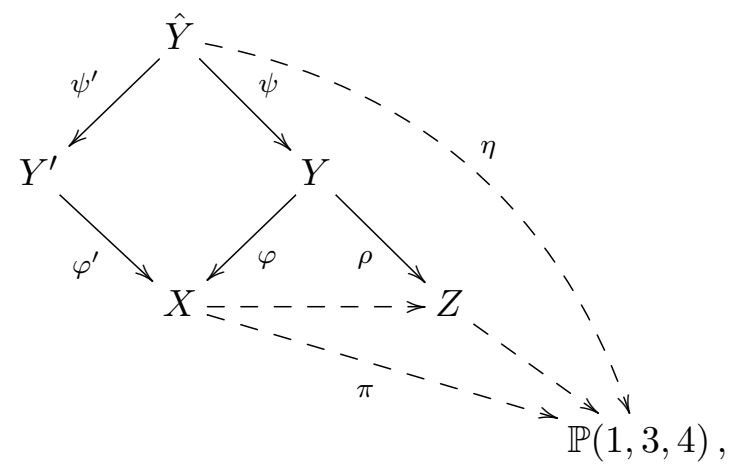

where $\psi^{\prime}: \hat{Y} \rightarrow Y^{\prime}$ is the Kawamata blowup of $Y^{\prime}$ at the $\frac{1}{5}(1,2,3)$-point $\varphi^{\prime-1}(\mathrm{p})$ and $\eta$ is the rational map induced by $\pi$. Note that the exceptional divisors of $\psi$ and $\psi^{\prime}$ are $\hat{E}^{\prime}$ and $\hat{E}$, which are the proper transforms of $E^{\prime}$ and $E$, respectively, where we recall that $E^{\prime}$ is the exceptional divisor of the Kawamata blowup $\varphi^{\prime}: Y^{\prime} \rightarrow X$ at the $\frac{1}{5}(1,1,4)$-point $\mathrm{q}=\mathrm{p}_{t_{0}}$. We set $B=-K_{Y}$ and $\hat{B}=-K_{\hat{Y}}$. It is straightforward to compute that $\left(B^{3}\right)=1 / 20$ and $\left(\hat{B}^{3}\right)=0$.

LEMma 7.15. The map $\eta$ is a morphism which is an elliptic fibration. Moreover, $\hat{E}$ and $\hat{E}^{\prime}$ are, respectively, 2- and 3-sections of $\eta$.

Proof. The indeterminacy locus of the projection $\pi: X \rightarrow \mathbb{P}(1,3,4)$ is the set $\Xi:=(x=y=$ $z=0) \cap X$. We have

$$
F_{1}\left(0,0,0, t_{0}, t_{1}, u, v\right)=t_{1} t_{0}, \quad F_{2}\left(0,0,0, t_{0}, t_{1}, u, v\right)=t_{1} u,
$$

so that $\Xi=\Xi_{1} \cup \Xi_{2}$, where

$$
\Xi_{1}=\left(x=y=z=t_{1}=0\right) \cap X, \quad \Xi_{2}=\left(x=y=z=t_{0}=u=0\right) \cap X .
$$

By looking at the other polynomials $F_{3}, F_{4}$ and $F_{5}$, it is easy to check that $\Xi_{1}=\left\{\mathrm{p}_{t_{0}}\right\}$ and $\Xi_{2}=$ $\left\{\mathrm{p}_{t_{1}}\right\}$. This shows that $\pi$ is defined outside $\left\{\mathrm{p}_{t_{0}}, \mathrm{p}_{t_{1}}\right\}$. The proper transforms of the sections $x, y$ and $z$ on $Y$ restrict to the coordinates $x, y$ and $z$ on $E \subset \mathbb{P}$, and we have $(x=y=z=0) \cap E=\emptyset$. This shows that $\eta$ is defined at every point of $\hat{E}$. For $\lambda, \mu \in \mathbb{C}$, we set $S_{\lambda}=\left(y-\lambda x^{3}=0\right) \cap X$ and $T_{\mu}=\left(z-\mu x^{4}=0\right) \cap X$. We see that $\tilde{S}_{\lambda} \cap \tilde{T}_{\mu}$ is the fibre of $\pi \circ \varphi: Y \rightarrow \mathbb{P}(1,3,4)$ over the point $(1: \lambda: \mu)$, and that $\left.\tilde{S}_{\lambda}\right|_{E}$ and $\left.\tilde{T}_{\mu}\right|_{E}$ are hyperplane sections of degree 3 and 4 , respectively, on $E \subset \mathbb{P}$, so that we have

$$
\left(\tilde{S}_{\lambda} \cdot \tilde{T}_{\mu} \cdot E\right)=\left(\left.\left.\tilde{S}_{\lambda}\right|_{E} \cdot \tilde{T}_{\mu}\right|_{E}\right)_{E}=\frac{3 \cdot 4 \cdot 5 \cdot 6 \cdot 4}{1 \cdot 3 \cdot 4 \cdot 5 \cdot 6 \cdot 2}=2 .
$$

This shows that $\hat{E}$ is a 2-section of $\eta$. Let us explain this computation in more detail. Since $E$ is a complete intersection in $\mathbb{P}$ defined by equations of degree 5,6 and 4 and $\left.\tilde{S}_{\lambda}\right|_{E}$ and $\left.\tilde{T}_{\mu}\right|_{E}$ correspond to hypersurfaces in $\mathbb{P}$ of degree 3 and 4 , respectively, we have $\left(\left.\left.\tilde{S}_{\lambda}\right|_{E} \cdot \tilde{T}_{\mu}\right|_{E}\right)_{E}=3 \cdot 4 \cdot 5 \cdot 6 \cdot 4 \cdot\left(\mathcal{O}_{\mathbb{P}}(1)\right)^{5}$.

The proper transforms of the sections $x, y, z$ on $Y$ restrict to the coordinates $x, y, z$ on $E^{\prime} \subset \mathbb{P}^{\prime}$, and we have $(x=y=z=0) \cap E^{\prime}=\emptyset$. This shows that $\eta$ is defined at every point of $\hat{E}^{\prime}$. We see that $S_{\lambda}^{\prime} \cap T_{\lambda}^{\prime}$, where $S_{\lambda}^{\prime}=\psi_{*}^{\prime-1} S_{\lambda}$ and $T_{\mu}^{\prime}=\psi_{*}^{\prime-1} T_{\mu}$, is the fibre of $\varphi^{\prime} \circ \pi$ over the point $(1: \lambda: \mu)$ and that $\left.S_{\lambda}^{\prime}\right|_{E^{\prime}}$ and $\left.T_{\mu}^{\prime}\right|_{E^{\prime}}$ are hyperplane sections of degree 3 and 4 , respectively, on $F \subset \mathbb{P}^{\prime}$, so that we have

$$
\left(S_{\lambda}^{\prime} \cdot T_{\mu}^{\prime} \cdot E^{\prime}\right)=\left(\left.\left.S_{\lambda}^{\prime}\right|_{E^{\prime}} \cdot T_{\mu}^{\prime}\right|_{E^{\prime}}\right)_{E^{\prime}}=\frac{3 \cdot 4 \cdot 5 \cdot 2 \cdot 3}{1 \cdot 3 \cdot 4 \cdot 5 \cdot 1 \cdot 2}=3
$$




\section{H. Ahmadinezhad And T. OKada}

This shows that $\hat{E}^{\prime}$ is a 3 -section of $\eta$. We note that the intersections $\tilde{S}_{\lambda} \cap \tilde{T}_{\mu} \cap E$ and $S_{\lambda}^{\prime} \cap T_{\mu}^{\prime} \cap E$ can also be computed explicitly using local coordinates.

Thus $\hat{\pi}$ is everywhere defined. It is clear that the sections $x, y, z$ lift to sections of $\hat{B}, 3 \hat{B}, 4 \hat{B}$ respectively, so that $\eta$ is the anticanonical morphism and it is an elliptic fibration.

By Lemma 7.14, the map $\rho$ is a flopping contraction. Let $\tau=\tau_{0}: Y \rightarrow Y_{1}$ be the flop of $\rho$. Then $Y_{1}$ admits a $K_{Y_{1}}$-negative extremal ray because otherwise $K_{Y_{1}}$ is nef and big, which is impossible. There are 3 options: $Y_{1}$ is a Mori fibre space, $Y_{1}$ admits a $K_{Y_{1}}$-negative divisorial contractions to a $\mathbb{Q}$-Fano 3-fold or $Y_{1}$ admits a flip $Y_{1} \rightarrow Y_{2}$. In the last case, $Y_{2}$ also has the same options since $K_{Y_{2}}$ is not nef and big. Thus the flop $Y \rightarrow Y_{1}$ followed by a sequence of flips gives a 2-ray game which ends with a Mori fibre space; that is, we have a Sarkisov link $\sigma: X \rightarrow \bar{X} / \bar{S}$ to a Mori fibre space. We will show that $\bar{X}$ is not isomorphic to $X$, which requires all the results of this section.

Theorem 7.16. The Sarkisov link $\sigma$ starting with the Kawamata blowup of $X$ at the $\frac{1}{5}(1,2,3)$ point $\mathrm{p}$ is a link to a Mori fibre space which is not isomorphic to $X$. In particular, $X$ is not birationally rigid.

Proof. We assume $\bar{X} \cong X$. Then the link $\sigma$ sits in the diagram

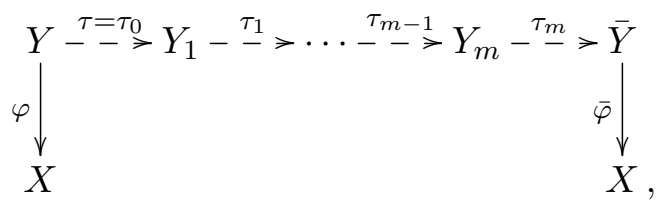

where $\tau_{i}$ is a flip for $i \geqslant 1$ and $\bar{\varphi}$ is an extremal divisorial contraction. We see that $\bar{\varphi}$ coincides with either $\varphi$ or $\varphi^{\prime}$ because a centre other than $\mathrm{p}$ and $\mathrm{p}_{t_{0}}$ is not a maximal centre. By Proposition 7.8 (see also [Oka17, Lemma 3.2]), the Sarkisov link starting with $\varphi^{\prime}$ ends with $\varphi^{\prime}$. By the uniqueness of a 2-ray game starting with a given divisorial extraction, $\bar{\varphi}$ cannot be $\varphi^{\prime}$ and hence $\bar{\varphi}=\varphi$. Now $\bar{Y} \cong Y$, so that it does not admit an inverse flip, which implies that $\tau_{m}$ cannot be a flip. Thus $m=0$, that is, the link involves only the flop $\tau$.

We have the following diagram:

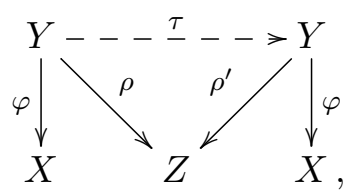

where $\rho^{\prime}$ is a flopping contraction. Note that $\rho^{\prime}$ can be decomposed as $\rho^{\prime}=\theta \circ \rho$, where $\theta: Z \rightarrow Z$ is an automorphism, since $\tau$ induces an isomorphism between the anticanonical model $Z$ of $Y$. Let $\hat{\tau}: \hat{Y} \rightarrow \hat{Y}$ be the birational automorphism induced by $\tau$. We set $N=\hat{B}+\varepsilon \hat{E}^{\prime}$ for $0<\varepsilon<\frac{1}{5}$, which is nef and big since $\psi^{*} B=\hat{B}+\frac{1}{5} \hat{E}^{\prime}$ is nef and big and $\hat{B}$ is nef. We choose $0<\varepsilon \ll \frac{1}{5}$ such that $N$ is $\psi$-ample. Let $\hat{\rho}: \hat{Y} \rightarrow \hat{Z}$ be the contraction associated with $N$.

We will show that the curves contracted by $\hat{\rho}$ are precisely the proper transforms of the flopping curves on $Y$. Let $\Gamma \subset Y$ be a flopping curve. Then

$$
0 \leqslant(\hat{B} \cdot \hat{\Gamma})=(B \cdot \Gamma)-\frac{1}{5}\left(\hat{E}^{\prime} \cdot \hat{\Gamma}\right)=-\frac{1}{5}\left(\hat{E}^{\prime} \cdot \hat{\Gamma}\right) \leqslant 0 .
$$

This shows $\hat{\Gamma} \cap \hat{E}^{\prime}=\emptyset$ and $(\hat{B} \cdot \hat{\Gamma})=0$. In particular, $\hat{\Gamma}$ is contracted by $\hat{\rho}$. Let $\Delta \subset \hat{Y}$ be an 


\section{Birationally Rigid Pfaffian Fano 3-Folds}

irreducible curve on $\hat{Y}$ which is contracted by $\hat{\rho}$. Note that $\Delta \not \subset \hat{E}^{\prime}$ since $N$ is $\psi$-ample. Then

$$
0=(N \cdot \Delta)=(\hat{B} \cdot \Delta)+\varepsilon\left(\hat{E}^{\prime} \cdot \Delta\right) \geqslant \varepsilon\left(\hat{E}^{\prime} \cdot \Delta\right) \geqslant 0,
$$

which implies $\Delta \cap \hat{E}^{\prime}=\emptyset$ and $0=(\hat{B} \cdot \Delta)=\left(B \cdot \psi_{*} \Delta\right)$. Thus $\Delta$ is the proper transform of a flopping curve on $Y$.

By the above argument, the curves contracted by $\hat{\rho}$ form a $K_{\hat{Y}}$-trivial extremal ray and $\hat{\rho}$ is a flopping contraction over $\mathbb{P}(1,3,4)$. Moreover, $\hat{Z}$ is obtained as the Kawamata blowup of $Z$ at the $\frac{1}{5}(1,1,4)$-point $\overline{\mathbf{q}}:=\rho\left(\varphi^{-1}(\mathbf{q})\right)$. Since the point $\varphi^{-1}(\mathbf{q}) \in Y$ is the unique singular point of type $\frac{1}{5}(1,1,4)$, the point $\overline{\mathrm{q}} \in Z$ is the unique point of type $\frac{1}{5}(1,1,4)$. Hence $\theta$ fixes $\overline{\mathrm{q}}$. It follows that the birational map $\hat{\tau}: \hat{Y} \rightarrow \hat{Y}$ is the flop of $\hat{\rho}$, and we have the following commutative diagram:

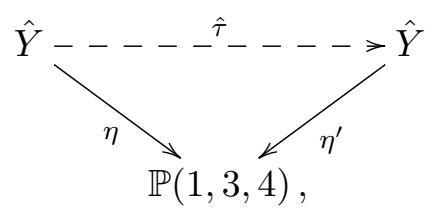

where $\eta^{\prime}=\chi \circ \eta$ for some automorphism $\chi$ of $\mathbb{P}(1,3,4)$ since the flop $\hat{\tau}$ induces an isomorphism of the anticanonical model $\mathbb{P}(1,3,4)$ of $\hat{Y}$. Thus $\hat{\tau}$ is an isomorphism in codimension 1 and it induces an isomorphism between the generic fibres of $\eta$ and $\eta^{\prime}$.

We have $\hat{\tau}_{*} \hat{B}=\hat{B}$ since $\hat{\tau}$ is small. By construction, we have $\hat{\tau}_{*} \hat{E}^{\prime}=\hat{E}^{\prime}$ (because $\theta(\overline{\mathrm{q}})=\overline{\mathrm{q}}$ ). Since the Weil divisor class group of $\hat{Y}$ is generated by $\hat{B}, \hat{E}$ and $\hat{E}^{\prime}$, we can write $\hat{\tau}_{*} \hat{E}=$ $\alpha \hat{B}-\beta \hat{E}+\gamma \hat{E}^{\prime}$ for some integers $\alpha, \beta, \gamma$. Clearly, $\alpha \geqslant 0$ since $\hat{\tau}_{*} \hat{E}$ is effective and non-zero. Note that $\tau_{*} E=\alpha B-\beta E$, and since $\tau$ is a flop, we have $\beta>0$. If $\alpha=0$, then $\tau_{*} E=-\beta E$ and this gives a contradiction since $\tau_{*} E$ is effective. Hence $\alpha>0$. We have

$$
\left(\hat{\tau}^{2}\right)_{*} \hat{E}=\alpha(1-\beta) \hat{B}+\beta^{2} \hat{E}+\gamma(1-\beta) \hat{F} .
$$

Since $\left(\hat{\tau}^{2}\right)_{*} \hat{E}$ is effective, we have $\alpha(1-\beta) \geqslant 0$, which implies $\beta \leqslant 1$. Thus we have $\beta=1$. Since $\hat{\tau}$ induces an isomorphism between generic fibres of the elliptic fibrations $\eta$ and $\eta^{\prime}$, the divisor $\hat{\tau}_{*} \hat{E}$ is a 2 -section of $\eta^{\prime}$. Clearly, $\hat{E}$ and $\hat{E}^{\prime}$ are 2- and 3-sections, respectively. Then, for a general $\eta^{\prime}$-fibre $C^{\prime}$, we have

$$
2=\left(\hat{\tau}_{*} \hat{E} \cdot C^{\prime}\right)=\alpha\left(\hat{B} \cdot C^{\prime}\right)-\left(\hat{E} \cdot C^{\prime}\right)+\gamma\left(\hat{E}^{\prime} \cdot C^{\prime}\right)=-2+3 \gamma .
$$

This gives a contradiction since $\gamma \in \mathbb{Z}$. Therefore, $\sigma$ cannot be a birational automorphism of $X$.

Remark 7.17. We are unable to give an explicit construction of the link $\sigma$, and we do not even understand whether the target Mori fibre space $\bar{X} / \bar{S}$ is a strict Mori fibre space or not.

\section{Pfaffian Fano 3-fold of degree 1/4}

Let $X=X_{7,8,8,9,10} \subset \mathbb{P}\left(1_{x}, 2_{y}, 3_{z_{0}}, 3_{z_{1}}, 4_{t_{0}}, 4_{t_{1}}, 5_{u}\right)$ be a Pfaffian Fano 3 -fold of degree $1 / 4$. The main aim of this section is to prove that there is a Sarkisov link centred at the $\frac{1}{4}(1,1,3)$-point to a Mori fibre space other than $X$. This implies that $X$ is not birationally rigid. For a rigorous proof, we need to exclude or untwist the other centres, so we will exclude points of type $\frac{1}{2}(1,1,1)$ and construct a Sarkisov link centred at each $\frac{1}{3}(1,1,2)$-point which is a birational involution. 


\section{H. Ahmadinezhad And T. OKada}

The syzygy matrix of $X$ and the defining polynomials are given as follows:

$$
M=\left(\begin{array}{ccccc}
0 & a_{2} & a_{3} & a_{3}^{\prime} & a_{4} \\
& 0 & b_{4} & b_{4}^{\prime} & b_{5} \\
& & 0 & c_{5} & c_{6} \\
& & 0 & d_{6} \\
& & & & 0
\end{array}\right), \quad \begin{aligned}
& F_{1}=a_{2} c_{5}-a_{3} b_{4}^{\prime}+a_{3}^{\prime} b_{4}, \\
& F_{2}=a_{2} c_{6}-a_{3} b_{5}+a_{4} b_{4}, \\
& F_{3}=a_{2} d_{6}-a_{3}^{\prime} b_{5}+a_{4} b_{4}^{\prime}, \\
& F_{4}=a_{3} d_{6}-a_{3}^{\prime} c_{6}+a_{4} c_{5}, \\
& F_{5}=b_{4} d_{6}-b_{4}^{\prime} c_{6}+b_{5} c_{5} .
\end{aligned}
$$

The basket of singularities of $X$ is

$$
\left\{3 \times \frac{1}{2}(1,1,1), 3 \times \frac{1}{3}(1,1,2), \frac{1}{4}(1,1,3)\right\} .
$$

\subsection{Exclusion of the $\frac{1}{2}(1,1,1)$-points}

Let $\mathrm{p}$ be a point of type $\frac{1}{2}(1,1,1)$. Throughout the present subsection, we assume $y \in a_{2}$ and then, re-scaling $y$, we may assume that the coefficient of $y$ in $a_{2}$ is 1 . Replacing $y, t_{0}, t_{1}$, we assume $\mathrm{p}=\mathrm{p}_{y}$. We have $u^{2} \in F_{5}$ since $\mathrm{p}_{u} \notin X$, which implies $u \in b_{5}, c_{5}$. It follows that $y u \in F_{1}$. After replacing $u$, we may assume that $y u$ is the unique monomial in $F_{1}$ which is divisible by $y$.

For the entries of the syzygy matrix $M$, we can write $a_{3}=\ell_{1}+$ (other terms), $a_{3}^{\prime}=\ell_{2}+$ (other terms), $b_{5}=y \ell_{3}+$ (other terms), $c_{5}=y \ell_{4}+$ (other terms), $c_{6}=\delta y^{3}+q_{1}+$ (other terms) and $d_{6}=\varepsilon y^{3}+q_{2}+$ (other terms), where $\delta, \varepsilon \in \mathbb{C}, \ell_{1}, \ldots, \ell_{4}$ and $q_{1}, q_{2}$ are, respectively, linear and quadratic forms in $t_{0}$ and $t_{1}$. Let $\beta, \gamma \in \mathbb{C}$ be the coefficients of $y^{2}$ in $b_{4}$ and $b_{4}^{\prime}$, respectively. We exclude the point $\mathrm{p}$ assuming the following generality condition.

Condition 8.1. We have $y \in a_{2}$ and the system of equations

$$
q_{1}-\ell_{1} \ell_{3}=q_{2}-\ell_{2} \ell_{3}=\beta q_{2}-\gamma q_{1}+\ell_{3} \ell_{4}=0
$$

does not have a non-trivial solution.

Lemma 8.2. If $X$ satisfies Condition 8.1, then no singular point of type $\frac{1}{2}(1,1,1)$ is a maximal centre.

Proof. We will prove that the set $\left\{x, t_{0}, t_{1}, u\right\}$ isolates $\mathrm{p}$. We set $\Pi=\left(x=t_{0}=t_{1}=u=0\right)$. Then we can write

$$
\left.M\right|_{\Pi}=\left(\begin{array}{ccccc}
0 & y & \ell_{1} & \ell_{2} & \alpha y^{2} \\
& 0 & \beta y^{2} & \gamma y^{2} & y \ell_{3} \\
& & 0 & y \ell_{4} & \delta y^{3}+q_{1} \\
& & & 0 & \varepsilon y^{3}+q_{2} \\
& & & & 0
\end{array}\right),
$$

where $\alpha, \beta, \ldots, \varepsilon \in \mathbb{C}$ and $\ell_{i}$ and $q_{i}$ are polynomials in $z_{0}$ and $z_{1}$ which are linear and quadratic, respectively. Hence we have

$$
\begin{aligned}
& \left.F_{1}\right|_{\Pi}=y^{2}\left(\ell_{4}-\gamma \ell_{1}+\beta \ell_{2}\right), \\
& \left.F_{2}\right|_{\Pi}=(\delta+\alpha \beta) y^{4}+y\left(q_{1}-\ell_{1} \ell_{3}\right), \\
& \left.F_{3}\right|_{\Pi}=(\varepsilon+\alpha \gamma) y^{4}+y\left(q_{2}-\ell_{2} \ell_{3}\right), \\
& \left.F_{4}\right|_{\Pi}=y^{3}\left(\varepsilon \ell_{1}-\delta \ell_{2}+\alpha \ell_{4}\right)+\ell_{1} q_{2}-\ell_{2} q_{1}, \\
& \left.F_{5}\right|_{\Pi}=(\beta \varepsilon-\gamma \delta) y^{5}+y^{2}\left(\beta q_{2}-\gamma q_{1}+\ell_{3} \ell_{4}\right) .
\end{aligned}
$$

By our choice of coordinates, there is no monomial in $F_{1}$ divisible by $y$ other than $y u$, so that $\ell_{4}-\gamma \ell_{1}+\beta \ell_{2}=0$. Since $\mathrm{p}=\mathrm{p}_{y} \in X$, we see that the coefficients of $y^{4}, y^{4}$ and $y^{5}$ in $F_{2}, F_{3}$ 


\section{Birationally Rigid Pfaffian Fano 3-Folds}

and $F_{5}$, respectively, are zero, which implies

$$
\delta+\alpha \beta=\varepsilon+\alpha \gamma=\beta \varepsilon-\gamma \delta=0 .
$$

Combining the above observations, we have

$$
\varepsilon \ell_{1}-\delta \ell_{2}+\alpha \ell_{4}=(\varepsilon+\alpha \gamma) \ell_{1}-(\delta+\alpha \beta) \ell_{2}=0,
$$

hence $\left.F_{1}\right|_{\Pi}=0$ and

$$
\begin{array}{ll}
\left.F_{2}\right|_{\Pi}=y\left(q_{1}-\ell_{1} \ell_{3}\right), & \left.F_{3}\right|_{\Pi}=y\left(q_{2}-\ell_{2} \ell_{3}\right), \\
\left.F_{4}\right|_{\Pi}=\ell_{1} q_{2}-\ell_{2} q_{1}, & \left.F_{5}\right|_{\Pi}=y^{2}\left(\beta q_{2}-\gamma q_{1}+\ell_{3} \ell_{4}\right) .
\end{array}
$$

By Condition 8.1, the intersection $X \cap \Pi$ consists of $\mathrm{p}$ and the 3 points of type $\frac{1}{3}(1,1,2)$. Thus $\left\{x, t_{0}, t_{1}, u\right\}$ isolates $\mathrm{p}$.

We see that $y^{3} x, y^{2} z_{0}, y^{2} z_{1}$ and $y u$ are the monomials of degree 7 having initial weight $\frac{1}{2}$. By our choice of coordinates, $y u$ is the unique monomial with initial weight $\frac{1}{2}$. It follows that $\operatorname{ord}_{E}\left(x, t_{0}, t_{1}, u\right) \geqslant \frac{1}{2}(1,2,2,3)$. Hence $L=4 \varphi^{*} A-\frac{2}{2} E$ is nef by Lemma 3.3 and we compute

$$
\left(L \cdot B^{2}\right)=4\left(A^{3}\right)-\frac{2}{2^{3}}\left(E^{3}\right)=\frac{4}{4}-\frac{2}{2}=0 .
$$

Therefore, $\mathrm{p}$ is not a maximal centre by Lemma 3.1.

\subsection{The $\frac{1}{3}(1,1,2)$-points and birational involutions}

Let $\mathrm{p} \in X$ be a point of type $\frac{1}{3}(1,1,2)$. For a polynomial $f=f\left(x, y, z_{0}, z_{1}, t_{0}, t_{1}, u\right)$, we write $\bar{f}=f\left(0,0, z_{0}, z_{1}, t_{0}, t_{1}, 0\right)$. Note that, for the entries $a_{3}, a_{3}^{\prime}$ and $a_{4}, b_{4}, b_{4}^{\prime}$ of the syzygy matrix of $X$, the polynomials $\bar{a}_{3}, \bar{a}_{3}^{\prime}$ and $\bar{a}_{4}, \bar{b}_{4}, \bar{b}_{4}^{\prime}$ are linear forms in $z_{0}, z_{1}$ and $t_{0}, t_{1}$, respectively. Note also that $\bar{c}_{6}$ and $\bar{d}_{6}$ are quadratic forms in $z_{0}, z_{1}$.

CONDition 8.3. The set

$$
\left(-\bar{a}_{3} \bar{b}_{4}^{\prime}+\bar{a}_{3}^{\prime} \bar{b}_{4}=\bar{a}_{3} \bar{d}_{6}-\bar{a}_{3}^{\prime} \bar{c}_{6}=\bar{a}_{4}=0\right) \subset \mathbb{P}\left(3_{z_{0}}, 3_{z_{1}}\right) \times \mathbb{P}\left(4_{t_{0}}, 4_{t_{1}}\right)
$$

is empty.

It is clear that Condition 8.3 is satisfied for a general $X$, and we assume that $X$ satisfies it.

Remark 8.4. Let $X$ be a Paffian Fano 3-fold defined by the syzygy matrix

$$
M=\left(\begin{array}{cccc}
a_{2} & a_{3} & a_{3}^{\prime} & a_{4} \\
0 & b_{4} & b_{4}^{\prime} & b_{5} \\
& 0 & c_{5} & c_{6} \\
& & 0 & d_{6}
\end{array}\right)
$$

and let $F_{1}, \ldots, F_{5}$ be defining polynomials. For $\alpha \in \mathbb{C}$, the matrices

$$
M_{\alpha}=\left(\begin{array}{cccc}
a_{2} & a_{3}-\alpha a_{3}^{\prime} & a_{3}^{\prime} & a_{4} \\
0 & b_{4}-\alpha b_{4}^{\prime} & b_{4}^{\prime} & b_{5} \\
& 0 & c_{5} & c_{6}-\alpha d_{6} \\
& & 0 & d_{6}
\end{array}\right), \quad M_{\alpha}^{\prime}=\left(\begin{array}{cccc}
a_{2} & a_{3} & a_{3}^{\prime}-\alpha a_{3} & a_{4} \\
0 & b_{4} & b_{4}^{\prime}-\alpha b_{4} & b_{5} \\
& 0 & c_{5} & c_{6} \\
& 0 & d_{6}-\alpha c_{6}
\end{array}\right)
$$

both define the same Pfaffian 3 -fold $X$ with defining polynomials $F_{1}, F_{2}-\alpha F_{3}, F_{3}, F_{4}, F_{5}$ and $F_{1}, F_{2}, F_{3}-\alpha F_{2}, F_{4}, F_{5}$, respectively.

The following choice of coordinates will also be used in the next subsection. 


\section{H. Ahmadinezhad And T. OKada}

Lemma 8.5. Let $\mathrm{p} \in X$ be a point of type $\frac{1}{3}(1,1,2)$ and $\mathrm{q} \in X$ the point of type $\frac{1}{4}(1,1,3)$. By a choice of coordinates, we can assume $\mathrm{p}=\mathrm{p}_{z_{1}}$ and $\mathrm{q}=\mathrm{p}_{t_{1}}$ and that the polynomials $F_{1}, \ldots, F_{5}$ are written as follows:

$$
\begin{aligned}
& F_{1}=t_{1} z_{1}+u a_{2}+t_{0} a_{3}+a_{7} \\
& F_{2}=t_{1} t_{0}+u b_{3}+t_{0} b_{4}+z_{1} b_{5}+b_{8}, \\
& F_{3}=z_{1} u+u c_{3}+t_{0}^{2}+t_{0} c_{4}+t_{1} g_{4}+z_{1} c_{5}+c_{8}, \\
& F_{4}=z_{1}^{2} z_{0}+u t_{0}+u g_{4}^{\prime}+t_{0} g_{5}+t_{1} g_{5}^{\prime}+z_{1} d_{6}+d_{9}, \\
& F_{5}=t_{1}^{2} y+t_{1}\left(u e_{1}+h_{6}\right)+u^{2}+u h_{5}+h_{10},
\end{aligned}
$$

where $a_{i}, b_{i}, c_{i}, d_{i}, e_{i} \in \mathbb{C}\left[x, y, z_{0}\right], g_{i} \in \mathbb{C}\left[x, y, z_{0}, z_{1}\right]$ and $h_{i} \in \mathbb{C}\left[x, y, z_{0}, z_{1}, t_{0}\right]$ are all contained in the ideal $\left(x, y, z_{0}\right)$ and satisfy $z_{0} \in b_{3}$ and $z_{1}^{3} x \notin h_{10}$.

Proof. We have $u \in b_{5}, c_{5}$ since $u^{2} \in F_{5}$ by the quasi-smoothness of $X$. The equation $\bar{a}_{4} \bar{b}_{4}=$ $\bar{a}_{4} \bar{b}_{4}^{\prime}=0$ has a unique non-trivial solution, which corresponds to the $\frac{1}{4}(1,1,3)$-point of $X$. It follows that $\bar{b}_{4}=\bar{b}_{4}^{\prime}=0$ has no non-trivial solution, and the solution $\bar{a}_{4}=0$ corresponds to the $\frac{1}{4}(1,1,3)$-point. We choose coordinates such that $\mathrm{p}=\mathrm{p}_{z_{1}}$ and $\mathrm{q}=\mathrm{p}_{t_{1}}$, which are equivalent to $z_{0} \mid\left(\bar{a}_{3} \bar{d}_{6}-\bar{a}_{3}^{\prime} \bar{c}_{6}\right)$ and $\bar{a}_{4}=t_{0}$. By suitable modifications of the matrix $M$ in Remark 8.4, we may assume $\bar{a}_{3}=z_{0}$. We have $t_{1} \in b_{4}$ because otherwise the set in Condition 8.3 contains the point $((0: 1),(0: 1))$, which is impossible. Again by a suitable modification of $M$, we may assume $\bar{b}_{4}^{\prime}=t_{0}$. Then, since neither $\bar{b}_{4}=\bar{b}_{4}^{\prime}=0$ nor $\bar{a}_{3}=\bar{a}_{3}^{\prime}=0$ has non-trivial solution, we have $t_{1} \in \bar{b}_{4}$ and $z_{1} \in a_{3}^{\prime}$. Replacing $t_{1}$ by $t_{1}-\varepsilon_{1} t_{0}$ and $z_{1}$ by $z_{1}-\varepsilon_{2} z_{0}$ for some $\varepsilon_{1}, \varepsilon_{2} \in \mathbb{C}$, we may assume $\bar{b}_{4}=t_{1}, \bar{a}_{4}=t_{0}$ and $\bar{a}_{3}^{\prime}=z_{1}$. So far, we have chosen coordinates such that $\mathrm{p}=\mathrm{p}_{z_{1}}$, $\mathrm{q}=\mathrm{p}_{t_{1}}, \bar{a}_{3}=z_{0}, \bar{a}_{3}^{\prime}=z_{1}, \bar{b}_{4}=t_{1}, \bar{b}_{4}^{\prime}=t_{0}$ and $z_{0} \mid \bar{c}_{6}$, where the last assertion follows from $z_{0} \mid\left(\bar{a}_{3} \bar{d}_{6}-\bar{a}_{3}^{\prime} \bar{c}_{6}\right)$ and $\bar{a}_{3}=z_{0}$.

We further replace coordinates while preserving the above properties. We replace $u$ in such a way that $c_{5}=u$. We replace $z_{0}$ by $h_{3}(x, y)$ and $z_{1}$ by $z_{1}-h_{3}^{\prime}(x, y)$ for suitable $h_{3}, h_{3}^{\prime} \in \mathbb{C}[x, y]$ such that $a_{3}=z_{0}$ and $a_{3}^{\prime}=z_{1}$. Now we can write the syzygy matrix $M$ as

$$
M=\left(\begin{array}{cccc}
a_{2} & z_{0} & z_{1} & t_{0}+A_{4} \\
0 & t_{1}+B_{4} & t_{0}+z_{1} b_{1}^{\prime}+b_{4}^{\prime} & \alpha u+t_{0} e_{1}+t_{1} e_{1}^{\prime}+B_{5} \\
& 0 & u & u c_{1}+t_{0} c_{2}+t_{1} c_{2}^{\prime}+C_{6} \\
& & 0 & u d_{1}+t_{0} d_{2}+t_{1} d_{2}^{\prime}+D_{6}
\end{array}\right),
$$

where $\alpha \in \mathbb{C} \backslash\{0\}, a_{2}, a_{3}, a_{3}^{\prime}, \ldots, d_{2}^{\prime}, e_{1}, e_{1}^{\prime} \in \mathbb{C}\left[x, y, z_{0}\right]$ and $A_{4}, B_{4}, B_{5}, C_{6}, D_{6} \in \mathbb{C}\left[x, y, z_{0}, z_{1}\right]$. We replace $t_{0}$ by $t_{0}-A_{4}-a_{2} c_{2}^{\prime}+e_{1}^{\prime} z_{0}$ so that, after the replacement, we have $A_{4}=-a_{2} c_{2}^{\prime}+e_{1}^{\prime} z_{0}$. We then replace $t_{1}$ by $t_{1}+b_{1}^{\prime} z_{0}-B_{4}$ so that, after the replacement, we have $B_{4}=b_{1}^{\prime} z_{0}$.

We claim $y \in d_{2}^{\prime}$. Indeed, since $\mathrm{q}=\mathrm{p}_{t_{1}}$ is of type $\frac{1}{4}(1,1,3)$, we have $t_{1}^{2} y \in F_{5}$. The terms in $F_{5}$ divisible by $t_{1}^{2}$ are computed as $t_{1}^{2} d_{2}^{\prime}$. Hence $y \in d_{2}^{\prime}$ and the claim is proved. We replace $y$ in such a way that $d_{2}^{\prime}=y$. We finish the choice of coordinates and in the following we observe that this is the desired choice of coordinates.

We compute $F_{1}, \ldots, F_{5}$. In the following descriptions, omitted terms $\cdots$ consist of monomials in the variables $x, y$ and $z_{0}$. We have

$$
\begin{aligned}
& F_{1}=t_{1} z_{1}+u a_{2}-t_{0} z_{0}+\cdots, \\
& F_{2}=t_{1} t_{0}+u\left(a_{2} c_{1}-\alpha z_{0}\right)+t_{0}\left(a_{2} c_{2}+b_{1}^{\prime} z_{0}\right)+a_{2} C_{6}-z_{0} B_{5}+\cdots, \\
& F_{3}=-\alpha z_{1} u+u a_{2} d_{1}+t_{0}^{2}+t_{0}\left(-z_{1} e_{1}+\cdots\right)+t_{1}\left(a_{2} d_{2}^{\prime}-z_{1} e_{1}^{\prime}\right)+a_{2} D_{6}-z_{1} B_{5}+\cdots, \\
& F_{4}=u\left(t_{0}-z_{1} c_{1}+\cdots\right)+t_{1}\left(z_{0} y-z_{1} c_{2}^{\prime}\right)+t_{0}\left(z_{0} d_{2}-z_{1} c_{2}\right)+z_{0} D_{6}-z_{1} C_{6} .
\end{aligned}
$$




\section{Birationally Rigid Pfaffian Fano 3-Folds}

Recall that $z_{0} \bar{D}_{6}-z_{1} \bar{C}_{6}=0$ has 3 distinct solutions (corresponding to 3 points of type $\frac{1}{3}(1,1,2)$ ) and, by our choice of coordinates, $z_{0} \mid z_{0} \bar{D}_{6}-z_{1} \bar{C}_{6}$. It follows that $z_{1}^{2} \notin C_{6}$ and $z_{1}^{2} \in D_{6}$. Thus, it is easy to see that $F_{1}, F_{2}$ and $F_{3}$ are in the form described in the statement after re-scaling $u$. We have $z_{1}^{2} z_{0} \in F_{4}=z_{0} D_{6}-z_{1} C_{6}$, which shows that $F_{4}$ is also in the desired form. Although we do not write down $F_{5}$ explicitly here, it is easy to verify that

$$
F_{5}=t_{1}^{2} y+t\left(u e_{1}+h_{6}\right)+\beta u^{2}+u h_{5}+h_{10}
$$

for some $\beta \in \mathbb{C} \backslash\{0\}, e_{i} \in \mathbb{C}\left[x, y, z_{0}\right]$ and $h_{i} \in \mathbb{C}\left[x, y, z_{0}, z_{1}, t_{0}\right]$. It is easy to observe that $h_{5}, h_{10} \in\left(x, y, z_{0}\right)$ because their degrees are not divisible by 3 , and neither can contain a power of $z_{1}$. This also explains that $g_{i}, g_{i}^{\prime} \in\left(x, y, z_{0}\right)$. Note that $h_{6}=D_{6}-b_{1}^{\prime} c_{2}^{\prime} z_{1}+\cdots$ and it contains $z_{1}^{2}$. By replacing $F_{5}$ by $F_{5}-\gamma z_{1} F_{1}$, we can eliminate the term $z_{1}^{2}$ in $h_{6}$. Finally, replacing $y$ by $\beta y$ and then replacing $F_{5}$ by $\frac{1}{\beta} F_{5}$, we may assume $\beta=1$. This completes the proof.

We choose and fix coordinates as above. It is easy to see that $z_{1} t_{1}$ is the unique monomial in $F_{1}=z_{1} t_{1}+u a_{2}+t_{0} a_{3}+a_{7}$ having initial weight $\frac{1}{3}$ since $a_{i}=a_{i}\left(x, y, z_{0}\right)$ has initial weight $\frac{i}{3}$. The Kawamata blowup $\varphi: Y \rightarrow X$ at $\mathrm{p}$ is realized as the embedded weighted blowup at $\mathrm{q}$ with weight $\operatorname{wt}\left(x, y, z_{0}, t_{0}, t_{1}, u\right)=\frac{1}{3}(1,2,3,1,4,2)=: \mathbf{w}$.

Let $\pi: X \rightarrow \mathbb{P}:=\mathbb{P}(1,2,3,4)$ be the projection to the coordinates $x, y, z_{0}, t_{1}$, and let $\pi_{Y}: Y \rightarrow \mathbb{P}(1,2,3,4)$ be the induced rational map. We take $H \in\left|\mathcal{O}_{\mathbb{P}}(1)\right|$.

Lemma 8.6. The map $\pi_{Y}$ is a surjective generically finite morphism of degree 2 such that $B=$ $\pi_{Y}^{*} H$.

Proof. By Lemma 8.5, it is easy to observe that the indeterminacy locus of $\pi$, which is the set $\left(x=y=z_{0}=t_{1}=0\right) \cap X$, consists of the single point $\mathrm{p}$ since $a_{i}, \ldots, e_{i}, g_{i}, g_{i}^{\prime}, h_{i}$ all vanish along $\left(x=y=z_{0}=0\right)$. We have an isomorphism

$$
E \cong\left(t_{1}+u a_{2}+t_{0} a_{3}=u+\alpha t_{0}^{2}+\gamma t_{0} x=z_{0}+u t_{0}+\delta u x=0\right) \subset \mathbb{P}\left(1_{x}, 2_{y}, 3_{z_{0}}, 1_{t_{0}}, 4_{t_{1}}, 2_{u}\right),
$$

where $\gamma, \delta \in \mathbb{C}$ are the coefficients of $t_{0} z_{1} x$ and $z_{1} x$ in $h_{8}$ and $g_{4}$, respectively. The sections $x, y$, $z_{0}, t_{1}$ lift to plurianticanonical sections on $Y$ and restrict to the coordinates $x, y, z_{0}, t_{1}$ of the ambient weighted projective space of $E$. It is clear that

$$
\left(x=y=z_{0}=t_{1}=0\right) \cap E=\emptyset
$$

since $\alpha \neq 0$. This shows that $\pi_{Y}$ is everywhere defined. We see $\pi_{Y}^{*} H=B$, and we compute $\left(H^{3}\right)=1 / 24$ and

$$
\left(B^{3}\right)=\left(A^{3}\right)-\frac{1}{3^{3}}\left(E^{3}\right)=\frac{1}{4}-\frac{1}{6}=\frac{1}{12} .
$$

From this, we see that $\pi_{Y}$ is surjective and has degree 2 .

By the same argument as in the proof of Proposition 6.9, the above lemma implies the following.

Proposition 8.7. One of the following holds:

(i) The point $\mathrm{p}$ is not a maximal centre.

(ii) There is a birational involution $\sigma: X \rightarrow X$ which is a Sarkisov link centred at $\mathrm{p}$.

\subsection{The $\frac{1}{4}(1,1,3)$-point and birational non-rigidity}

Let $\mathrm{p}$ be the point of type $\frac{1}{4}(1,1,3)$. We will show that the Kawamata blowup $\varphi: Y \rightarrow X$ leads to a Sarkisov link to a Mori fibre space which is not isomorphic to $X$. The arguments are similar 


\section{H. Ahmadinezhad And T. OKada}

to those in Section 7.4 but more complicated. Note that the $X$ has 3 points of type $\frac{1}{3}(1,1,2)$, denoted $\mathbf{q}_{1}, \mathbf{q}_{2}, \mathbf{q}_{3}$. We choose coordinates as in Lemma 8.5 for the $\frac{1}{3}(1,1,2)$-point $\mathbf{q}_{1}$ and the $\frac{1}{4}(1,1,3)$-point $\mathrm{p}$ such that $\mathrm{q}_{1}=\mathrm{p}_{z_{1}}$ and $\mathrm{p}=\mathrm{p}_{t_{1}}$.

Recall that Lemma 8.5 is based on Condition 8.3, which we assume in this subsection. In addition, we assume the following condition, which is satisfied for a general $X$.

CONDition 8.8. Under the choice of coordinates as in Lemma 8.5, we have $y \in a_{2}$ and the set

$$
\left(a_{2}=b_{3}=y+u e_{1}+u^{2}=0\right) \subset \mathbb{P}\left(1_{x}, 2_{y}, 3_{z_{0}}, 1_{u}\right)
$$

consists of 2 distinct points.

The Kawamata blowup $\varphi: Y \rightarrow X$ at $\mathrm{p}$ is realized as the embedded weighted blowup with the initial weight $\mathrm{wt}\left(x, y, z_{0}, z_{1}, t_{0}, u\right)=\mathbf{w}_{\text {in }}=\frac{1}{4}(1,2,3,3,4,1)$, and we have an isomorphism

$$
E \cong\left(z_{1}+u a_{2}=t_{0}+u b_{3}=y+u e_{1}+u^{2}=0\right) \subset \mathbb{P},
$$

where $\mathbb{P}=\mathbb{P}\left(1_{x}, 2_{y}, 3_{z_{0}}, 3_{z_{1}}, 4_{t_{0}}, 1_{u}\right)$. Let $X \rightarrow \mathbb{P}(1,2,3,3,4)$ be the projection to $x, y, z_{0}, z_{1}, t_{0}$, and denote its image by $Z$. Let $\rho: Y \rightarrow Z$ be the induced map.

Lemma 8.9. The map $\rho$ is a flopping contraction.

Proof. By Lemma 8.5, it is easy to observe that the projection $X \rightarrow \mathbb{P}(1,2,3,3,4)$ is defined outside p. The sections $x, y, z_{0}, z_{1}, t_{0}$ lift to plurianticanonical sections on $Y$ and restrict to $E$ the coordinates $x, y, z_{0}, z_{1}, t_{0}$ of $\mathbb{P}$. We see

$$
\left(x=y=z_{0}=z_{1}=t_{0}=0\right) \cap E=\emptyset,
$$

which shows that $\rho$ is a morphism. By the same argument as in the proof Lemma 7.12, we see that $\rho$ is birational and is the anticanonical model of $Y$. The set $\left(a_{2}=b_{3}=0\right) \cap E$ consits of 2 points by Condition 8.8, which is mapped to the same point via $\rho$, which shows that $\rho$ is not an isomorphism.

It remains to show that $\rho$ is small. Assume that $\rho$ is divisorial, and let $G$ be the prime divisor on $Y$ contracted by $\rho$. Since $\left(B^{2} \cdot G\right)=0$, we have $G \sim_{\mathbb{Q}} m(2 B-E)$ for some positive integer $m$. By the same argument as in the proof of Lemma 7.14, the proper transform $\tilde{H}$ of the divisor $H$ on $X$ defined by $z_{1} b_{3}-t_{0} a_{2}=0$ satisfies $\tilde{H} \sim_{\mathbb{Q}} 6 B-E$. By Lemma 7.13, a component of $\tilde{H}$ which is $\mathbb{Q}$-linearly equivalent to $\lambda B-\mu E$ for some $\lambda, \mu$ with $\mu>0$ is $G$. It follows that $\tilde{H}$ contains $G$ as a component. This in particular implies $m \leqslant 2$. We see that $\varphi_{*} G \sim_{\mathbb{Q}} 2 m A$ is cut out on $X$ by a polynomial of degree $2 m$ with $2 m=2$ or 4 . Hence, $\varphi_{*} G$ contains the 3 singular points of type $\frac{1}{3}(1,1,2)$, and we conclude that $H$ contains the 3 singular points of type $\frac{1}{3}(1,1,2)$. But this is impossible since $H \sim_{\mathbb{Q}} 6 A$, and $6 A$ is defined by $z_{1} b_{3}-t_{0} a_{2}=0$ and contains at most 2 singular points of type $\frac{1}{3}(1,1,2)$. This gives a contradiction, and $\rho$ is a flipping contraction.

Let $\varphi_{1}^{\prime}: Y_{1}^{\prime} \rightarrow X$ be the Kawamata blowup at the $\frac{1}{3}(1,1,2)$-point $\mathrm{q}_{1}$ with exceptional divisor $E_{1}^{\prime}$. As is argued in the previous subsection, $\varphi_{1}^{\prime}$ is realized as the embedded weighted blowup at $\mathrm{q}_{1}=\mathrm{p}_{z_{1}}$ with weight $\operatorname{wt}\left(x, y, z_{0}, t_{0}, t_{1}, u\right)=\frac{1}{3}(1,2,3,1,4,2)$ and we have an isomorphism

$$
E_{1}^{\prime} \cong\left(t_{1}+u a_{2}+t_{0} a_{3}=u+t_{0}^{2}+\gamma t_{0} x=z_{0}+u t_{0}+\delta u x=0\right) \subset \mathbb{P}^{\prime}
$$

for some $\gamma, \delta \in \mathbb{C}$, where $\mathbb{P}^{\prime}=\mathbb{P}\left(1_{x}, 2_{y}, 3_{z_{0}}, 1_{t_{0}}, 4_{t_{1}}, 2_{u}\right)$.

Let $\psi_{1}: \hat{Y}_{1} \rightarrow Y$ be the Kawamata blowup of $Y$ at the $\frac{1}{3}(1,1,2)$-point $\varphi^{-1}\left(\mathbf{q}_{1}\right)$. We have a natural birational morphism $\psi_{1}^{\prime}: \hat{Y}_{1} \rightarrow Y_{1}^{\prime}$ which is the Kawamata blowup of the $\frac{1}{4}(1,1,3)$ point $\varphi_{1}^{\prime-1}(\mathrm{p})$. We see that the proper transforms $\hat{E}_{1}$ and $\hat{E}_{1}^{\prime}$ of $E$ and $E_{1}^{\prime}$ are the exceptional 


\section{Birationally Rigid Pfaffian Fano 3-Folds}

divisors of $\psi_{1}^{\prime}$ and $\psi_{1}$, respectively. We denote by $\pi_{1}: X \rightarrow \mathbb{P}(1,2,3)$ the projection to $x, y, z_{0}$ and by $\eta_{1}: \hat{Y} \rightarrow \mathbb{P}(1,2,3)$ the induced rational map. We set $B=-K_{Y}$ and $\hat{B}=-K_{\hat{Y}_{1}}$.

LEMma 8.10. The map $\eta_{1}$ is a morphism which is an elliptic fibration. Moreover, $\hat{E}_{1}$ and $\hat{E}_{1}^{\prime}$ are, respectively, 2- and 3-sections of $\eta_{1}$.

Proof. We first show that $\pi_{1}: X \rightarrow \mathbb{P}(1,2,3)$ is defined outside the set $\left\{\mathrm{q}_{1}, \mathrm{p}\right\}=\left\{\mathrm{p}_{z_{1}}, \mathrm{p}_{t_{1}}\right\}$. The indeterminacy locus of $\pi_{1}$ is the set $\Xi:=\left(x=y=z_{0}=0\right) \cap X$. We have

$$
F_{1}\left(0,0,0, z_{1}, t_{0}, t_{1}, u\right)=t_{1} z_{1}, \quad F_{2}\left(0,0,0, z_{1}, t_{0}, t_{1}, u\right)=t_{1} t_{0},
$$

so that $\Xi=\left(x=y=z_{0}=t_{1}=0\right) \cup\left(x=y=z_{0}=z_{1}=t_{1}=0\right)$. By looking at the other polynomials $F_{3}, F_{4}$ and $F_{5}$, it is easy to check that the former and the latter sets are $\left\{\mathrm{p}_{z_{1}}\right\}$ and $\left\{\mathrm{p}_{t_{1}}\right\}$, respectively, so that $\Xi=\left\{\mathrm{p}_{z_{1}}, \mathrm{p}_{t_{1}}\right\}$. It is straightforward to see $\left(x=y=z_{0}=0\right) \cap E=$ $\left(x=y=z_{0}=0\right) \cap E^{\prime}=\emptyset$, which shows that $\eta_{1}$ is a morphism. Since $x, y, z_{0}$ lift to sections of $\hat{B}, 2 \hat{B}, 3 \hat{B}$, respectively, $\eta_{1}$ is the anticanonical morphism of $\hat{Y}_{1}$, that is, it is an elliptic fibration.

For $\lambda, \mu \in \mathbb{C}$, we set $S_{\lambda}=\left(y-\lambda x^{2}=0\right) \cap X$ and $T_{\mu}=\left(z_{0}-\mu x^{3}=0\right) \cap X$. We see that $\tilde{S}_{\lambda} \cap \tilde{T}_{\mu}$, where $\tilde{S}_{\lambda}$ and $\tilde{T}_{\lambda}$ are the proper transforms of $S_{\lambda}$ and $T_{\mu}$ via $\varphi$, is the fibre of $\pi_{1} \circ \varphi: Y \rightarrow \mathbb{P}(1,2,3)$ over the point $(1: \lambda: \mu)$, and we compute

$$
\left(\tilde{S}_{\lambda} \cdot \tilde{T}_{\lambda} \cdot E\right)=\left(\left.\left.\tilde{S}_{\lambda}\right|_{E} \cdot \tilde{T}_{\mu}\right|_{E}\right)_{E}=\frac{2 \cdot 3 \cdot 3 \cdot 4 \cdot 2}{1 \cdot 2 \cdot 3 \cdot 3 \cdot 4 \cdot 1}=2 .
$$

Thus $\hat{E}_{1}$ is a 2-section of $\eta_{1}$. Similarly, $S_{\lambda}^{\prime} \cap T_{\mu}^{\prime}$, where $S_{\lambda}^{\prime}$ and $T_{\mu}^{\prime}$ are the proper transforms of $S_{\lambda}$ and $T_{\mu}$ via $\varphi_{1}^{\prime}$, is a fibre of $\pi_{1} \circ \varphi_{1}^{\prime}: Y_{1}^{\prime} \rightarrow \mathbb{P}(1,2,3)$ over the point $(1: \lambda: \mu)$, and we compute

$$
\left(S_{\lambda}^{\prime} \cdot T_{\lambda}^{\prime} \cdot E_{1}^{\prime}\right)=\left(\left.\left.S_{\lambda}^{\prime}\right|_{E_{1}^{\prime}} \cdot T_{\mu}^{\prime}\right|_{E_{1}^{\prime}}\right)_{E_{1}^{\prime}}=\frac{2 \cdot 3 \cdot 4 \cdot 2 \cdot 3}{1 \cdot 2 \cdot 3 \cdot 1 \cdot 4 \cdot 2}=3 .
$$

This shows that $\hat{E}_{1}^{\prime}$ is a 3 -section of $\eta_{1}$.

The above arguments hold true for $\mathrm{q}_{i}$, where $i=2,3$, instead of $\mathrm{q}_{1}$ (by re-choosing coordinates as in Lemma 8.5 for $\mathrm{q}_{i}$ and $\mathrm{p}$ ), and we obtain the following diagram for $i=1,2,3$ :

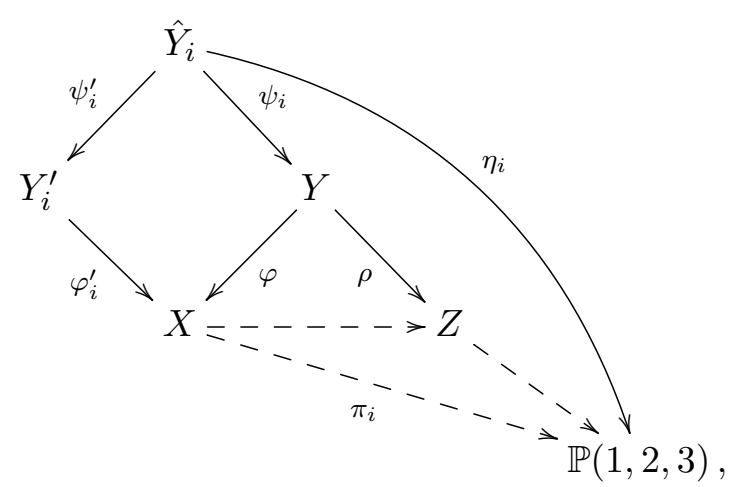

where $\varphi_{i}^{\prime}: Y_{i}^{\prime} \rightarrow X, \psi_{i}: \hat{Y}_{i} \rightarrow Y, \psi_{i}^{\prime}: \hat{Y}_{i} \rightarrow Y_{i}^{\prime}$ are the Kawamata blowups at $\mathbf{q}_{i} \in X, \varphi^{-1}\left(\mathbf{q}_{i}\right) \in Y$, $\varphi_{i}^{\prime-1}(\mathrm{p}) \in Y_{i}^{\prime}$, respectively, and $\eta_{i}: \hat{Y}_{i} \rightarrow \mathbb{P}(1,2,3)$ is the elliptic fibration induced by the natural projection $\pi_{i}: X \rightarrow \mathbb{P}(1,2,3)$. Let $E_{i}^{\prime}$ be the $\varphi_{i}^{\prime}$-exceptional divisor, and let $\hat{E}_{i}$ and $\hat{E}_{i}^{\prime}$ be the proper transforms of $E$ and $E_{i}^{\prime}$ via $\psi_{i}$ and $\psi_{i}^{\prime}$, respectively. By Lemma 8.10, the transforms $\hat{E}_{i}$ and $\hat{E}_{i}^{\prime}$ are a 2 - and 3 -sections of $\eta_{i}$, respectively.

TheOREM 8.11. The Sarkisov link $\sigma$ starting with the Kawamata blowup of $X$ at the $\frac{1}{4}(1,1,3)$ point is a link to a Mori fibre space which is not isomorphic to $X$. In particular, $X$ is not birationally rigid. 


\section{H. Ahmadinezhad And T. OKada}

Proof. Assume that the link $\sigma$ is a birational automorphism. Then, by the same argument as in the proof of Theorem 7.16, we obtain the flop $\tau$ of $\rho: X \rightarrow Z$, which is a birational automorphism sitting in a diagram

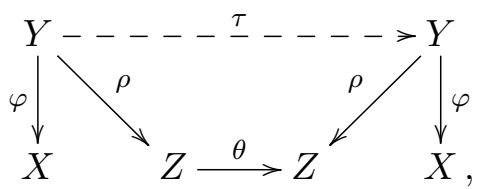

where $\theta$ is an automorphism. Note that $Y$ has 4 points of type $\frac{1}{3}(1,1,2)$, that is, $\varphi^{-1}\left(q_{i}\right)$ for $i=1,2,3$ and the point $\bar{q}$ on the exceptional divisor $E$. By the same argument as in the proof of Theorem 7.16, the curves contracted by $\rho$ do not pass through $\varphi^{-1}\left(\mathbf{q}_{i}\right)$ for $i=1,2,3$; hence $\rho$ is an isomorphism around $\varphi^{-1}\left(\mathbf{q}_{i}\right)$. We set $\overline{\mathbf{q}}_{i}=\rho\left(\varphi^{-1}\left(\mathbf{q}_{i}\right)\right) \in Z$, which is of type $\frac{1}{3}(1,1,2)$, and $\overline{\mathbf{q}}=\rho(\mathbf{q})$. Since $\theta$ is an automorphism, it maps a $\frac{1}{3}(1,1,2)$-point to a $\frac{1}{3}(1,1,2)$-point, and the set of $\frac{1}{3}(1,1,2)$-points on $Z$ is contained in $\left\{\overline{\mathbf{q}}_{1}, \ldots, \overline{\mathrm{q}}_{3}, \overline{\mathrm{q}}\right\}$. By renumbering, we may assume $\theta\left(\overline{\mathbf{q}}_{1}\right) \neq \overline{\mathbf{q}}$. Set $\theta\left(\overline{\mathbf{q}}_{1}\right)=\overline{\mathbf{q}}_{j}$ for $j \in\{1,2,3\}$.

For $i=1, j$, let $\hat{\rho}_{i}: \hat{Y}_{i} \rightarrow \hat{Z}_{i}$ be the morphism induced by $N_{i}=-K_{\hat{Y}_{i}}+\varepsilon \hat{E}_{i}^{\prime}$ for a sufficiently small $\varepsilon>0$. By the same argument as in the proof of Theorem 7.16, the morphism $\hat{\rho}_{i}$ is a flopping contraction and $\hat{Z}_{i}$ is obtained as the Kawamata blowup of $Z$ at $\overline{\mathrm{q}}_{i}$. Now, since $\theta\left(\overline{\mathrm{q}}_{1}\right)=\overline{\mathrm{q}}_{j}$, the automorphism $\theta: Z \rightarrow Z$ induces an isomorphism $\hat{\theta}: \hat{Z}_{1} \rightarrow \hat{Z}_{j}$, and we have the following diagram:

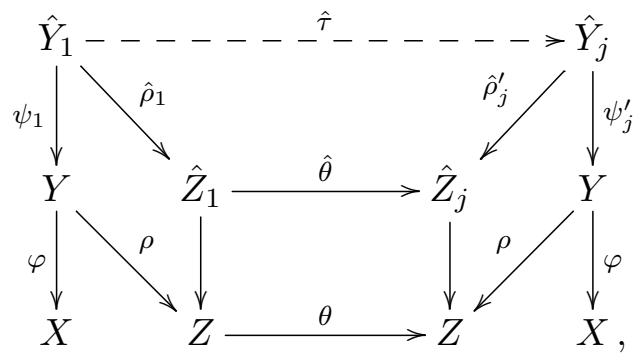

where $\hat{\tau}: \hat{Y}_{1} \rightarrow \hat{Y}_{j}$ is the map induced by $\tau: Y \rightarrow Y$. By construction, $\hat{\tau}_{*} \hat{E}_{1}^{\prime}=\hat{E}_{j}^{\prime}$. Hence $\hat{\tau}$ is an isomorphism in codimension 1; that is, it is a flop. By considering the anticanonical models of $\hat{Y}_{1}$ and $\hat{Y}_{j}$, we obtain an automorphism of $\mathbb{P}(1,2,3)$ sitting in the commutative diagram

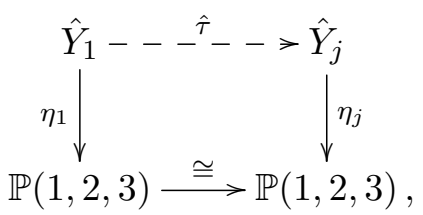

and $\hat{\tau}$ induces an isomorphism between generic fibres of the elliptic fibrations $\eta_{1}, \eta_{j}$.

We set $\hat{B}_{1}=-K_{\hat{Y}_{1}}$ and $\hat{B}_{j}=-K_{\hat{Y}_{j}}$. Then $\hat{\tau}_{*} \hat{B}_{1}=\hat{B}_{j}$ and $\hat{\tau}_{*} \hat{E}_{1}^{\prime}=\hat{E}_{j}^{\prime}$. We can write $\hat{\tau}_{*} \hat{E}_{1}=\alpha \hat{B}_{j}-\beta \hat{E}_{j}+\gamma \hat{E}_{j}^{\prime}$ for some integers $\alpha, \beta, \gamma$. Since $\hat{\tau}_{*}$ induces an isomorphism between the divisor class groups, we have

$$
\left(\begin{array}{ccc}
1 & 0 & 0 \\
\alpha & -\beta & \gamma \\
0 & 0 & 1
\end{array}\right) \in \mathrm{GL}_{3}(\mathbb{Z})
$$

which implies $\beta=1$. Since $\hat{\tau}_{*} \hat{E}_{1}, \hat{E}_{j}$ and $\hat{E}_{j}^{\prime}$ are 2-, 2- and 3 -sections of $\eta_{j}$, respectively, the computation of intersection numbers of $\hat{\tau}_{*} \hat{E}_{1}=\alpha \hat{B}_{j}-\hat{E}_{j}+\gamma \hat{E}_{j}^{\prime}$ and a general fibre $C$ of $\eta_{j}$ gives 


\section{Birationally Rigid Pfaffian Fano 3-Folds}

$\gamma=4 / 3$. This gives a contradiction since $\gamma$ is an integer, and the proof is completed.

\section{The table}

We summarize the result of this paper in the following table. The first column indicates the numbers and the types of singular points of $X$. The second column indicates the existence of a Sarkisov link centred at the corresponding point: if the second column is blank, then the corresponding point is not a maximal centre, and the mark "Q.I." or " $\exists$ Link" indicates that there is a Sarkisov link centred at the point which is a quadratic involution or a link to a Mori fibre space not isomorphic to $X$, respectively. The third column indicates the generality condition required to prove the result indicated in the second column.

$X_{16,17,18,19,20} \subset \mathbb{P}\left(1_{x}, 5_{y}, 6_{z}, 7_{t}, 8_{u}, 9_{v}, 10_{w}\right) ;\left(A^{3}\right)=1 / 42$.

\begin{tabular}{|c|c|c||c|c|c|}
\hline$\frac{1}{2}(1,1,1)$ & no & $\frac{1}{3}(1,1,2)$ & & no \\
\hline$\frac{1}{5}(1,1,4)$ & & Condition 4.5 & $\frac{1}{5}(1,2,3)$ & & no \\
\hline$\frac{1}{7}(1,1,6)$ & & no & & & \\
\hline
\end{tabular}

$X_{14,15,16,17,18} \subset \mathbb{P}\left(1_{x}, 5_{y}^{2}, 6_{z}, 7_{t}, 8_{u}, 9_{v}\right) ;\left(A^{3}\right)=1 / 30$.

\begin{tabular}{|l|c|c||c|c|c|}
\hline$\frac{1}{5}(1,1,4)$ & & Condition 5.6 & $2 \times \frac{1}{5}(1,2,3)$ & & no \\
\hline$\frac{1}{6}(1,1,5)$ & & no & & & \\
\hline
\end{tabular}

$X_{12,13,14,15,16} \subset \mathbb{P}\left(1_{x}, 4_{y}, 5_{z}^{2}, 6_{t}, 7_{u}, 8_{v}\right) ;\left(A^{3}\right)=1 / 20$.

\begin{tabular}{|l|l|l||l|l|l|}
\hline$\frac{1}{2}(1,1,1)$ & no & $\frac{1}{4}(1,1,3)$ & & Condition 6.4 \\
\hline $2 \times \frac{1}{5}(1,1,4)$ & & no & $\frac{1}{5}(1,2,3)$ & Q.I. & \\
\hline
\end{tabular}

$X_{10,11,12,13,14} \subset \mathbb{P}\left(1_{x}, 3_{y}, 4_{z}, 5_{t}^{2}, 6_{u}, 7_{v}\right) ;\left(A^{3}\right)=1 / 12$.

\begin{tabular}{|l|l|c||l|l|c|}
\hline $2 \times \frac{1}{3}(1,1,2)$ & & Condition 7.5 & $\frac{1}{4}(1,1,3)$ & & Condition 7.2 \\
\hline$\frac{1}{5}(1,1,4)$ & Q.I. & no & $\frac{1}{5}(1,2,3)$ & $\exists$ Link & Condition 7.11 \\
\hline
\end{tabular}

$X_{7,8,8,9,10} \subset \mathbb{P}\left(1_{x}, 2_{y}, 3_{z}^{2}, 4_{t}^{2}, 5_{u}\right) ;\left(A^{3}\right)=1 / 4$.

\begin{tabular}{|l|l|c||c|c|c|}
\hline $3 \times \frac{1}{2}(1,1,1)$ & & Condition 8.1 & $3 \times \frac{1}{3}(1,1,2)$ & Q.I. & Condition 8.3 \\
\hline$\frac{1}{4}(1,1,3)$ & $\exists$ Link & Conditions 8.3,8.8 & & & \\
\hline
\end{tabular}

\section{ACKNOWLEDGEMENTS}

We have benefitted from conversation with Gavin Brown, Ivan Cheltsov, Tommaso de Fernex, Miles Reid, Konstantin Shramov and Francesco Zucconi at various occasions. We would like to thank them for their generosity in sharing their knowledge and opinions with us.

\section{REFERENCES}

ABR02 S. Altınok, G. Brown and M. Reid, Fano 3-folds, K3 surfaces and graded rings, Topology and Geometry: Commemorating SISTAG, Contemp. Math., vol. 314 (Amer. Math. Soc., Providence, RI, 2002), 25-53; https://doi.org/10.1090/conm/314/05420.

AZ16 H. Ahmadinezhad and F. Zucconi, Mori dream spaces and birational rigidity of Fano 3-folds, Adv. Math. 292 (2016), 410-445; https://doi.org/10.1016/j. aim.2016.01.008. 


\section{H. Ahmadinezhad And T. OKada}

BE77 D. A. Buchsbaum and D. Eisenbud, Algebra structures for finite free resolutions, and some structure theorems for ideals of codimension 3, Amer. J. Math. 99 (1977), no. 3, 447-485; https://doi.org/10.2307/2373926.

BZ10 G. Brown and F. Zucconi, Graded rings of rank 2 Sarkisov links, Nagoya Math. J. 197 (2010), 1-44; https://doi.org/10.1215/00277630-2009-001.

CM04 A. Corti and M. Mella, Birational geometry of terminal quartic 3-folds. I, Amer. J. Math. 126 (2004), no. 4, 739-761, https://doi.org/10.1353/ajm.2004.0026.

CP17 I. Cheltsov and J. Park, Birationally rigid Fano threefold hypersurfaces, Mem. Amer. Math. Soc. 246 (2017), no. 1167; https://doi.org/10.1090/memo/1167.

CPR00 A. Corti, A. Pukhlikov and M. Reid, Fano 3-fold hypersurfaces, Explicit Birational Geometry of 3-Folds, London Math. Soc. Lecture Note Ser., vol. 281 (Cambridge Univ. Press, Cambridge, 2000), 175-258.

Dol82 I. Dolgachev, Weighted projective varieties, Group Actions and Vector Fields (Vancouver, B.C., 1981), Lecture Notes in Math., vol. 956 (Springer, Berlin, 1982), 34-71; https://doi.org/10. 1007/BFb0101508.

dF13 T. de Fernex, Birationally rigid hypersurfaces, Invent. Math. 192 (2013), no. 3, 533-566; https: //doi.org/10.1007/s00222-012-0417-0.

IM71 V.A. Iskovskih and Yu.I. Manin, Three-dimensional quartics and counterexamples to the Lüroth problem, Math. USSR Sb. 15 (1971), no. 1, 141-166; https://doi.org/10.1070/ SM1971v015n01ABEH001536.

IP96 V.A. Iskovskikh and A.V. Pukhlikov, Birational automorphisms of multidimensional algebraic manifolds, J. Math. Sci. 82 (1996), no. 4, 3528-3613; https://doi.org/10.1007/BF02363913.

Isk77 V. A. Iskovskih, Fano 3-folds. I, Math. USSR Izv. 11 (1977), no. 3, 485-527; https://doi.org/ 10.1070/IM1977v011n03ABEH001733.

Isk78_, Fano 3-folds. II, Math. USSR Izv. 12 (1978), no. 3, 469-506; https://doi.org/10. 1070/IM1978v012n03ABEH001994.

Kaw96 Y. Kawamata, Divisorial contractions to 3-dimensional terminal quotient singularities, HigherDimensional Complex Varieties (Trento, 1994) (de Gruyter, Berlin, 1996), 241-246; https: //doi.org/10.1515/9783110814736.241.

MM81 S. Mori and S. Mukai, Classification of Fano 3-folds with $B_{2} \geqslant 2$, Manuscripta Math. 36 (1981), no. 2, 147-162; https://doi.org/10.1007/BF01170131.

Oka14a T. Okada, Birational Mori fiber structures of $\mathbb{Q}$-Fano 3-fold weighted complete intersections, Proc. Lond. Math. Soc. 109 (2014), no. 6, 1549-1600; https://doi.org/10.1112/plms/pdu044.

Oka17 B Birational Mori fiber structures of $\mathbb{Q}$-Fano 3-fold weighted complete intersections, II, J. reine angew. Math., electronically published on 26 September 2015, https://doi.org/10. 1515/crelle-2015-0054, to appear in print.

Oka14b_ Birational Mori fiber structures of $\mathbb{Q}$-Fano 3-fold weighted complete intersections, III, 2014, arXiv:1409.1506.

Pap06 S. A. Papadakis, Type II unprojection, J. Algebraic Geom. 15 (2006), no. 3, 399-414; https: //doi.org/10.1090/S1056-3911-06-00433-4.

Pap08_- The equations of type $\mathrm{II}_{1}$ unprojection, J. Pure Appl. Algebra 212 (2008), no. 10, 21942208; https://doi.org/10.1016/j.jpaa.2008.03.016.

Puk98 A. Pukhlikov, Birational automorphisms of Fano hypersurfaces, Invent. Math. 134 (1998), no. 2, 401-426; https://doi.org/10.1007/s002220050269.

Puk13 , Birationally rigid varieties, Math. Surveys Monogr., vol. 190 (Amer. Math. Soc., Providence, RI, 2013); https://doi.org/10.1090/surv/190.

Rei00 M. Reid, Graded rings and birational geometry, Proceedings of Algebraic Geometry Symposium (Kinosaki, October 2000) (ed. K. Ohno; Kyoto University, 2000), 1-72; http://hdl.handle. net/2433/214728. 


\section{Birationally Rigid Pfaffian FAno 3-Folds}

Suz17 F. Suzuki, Birational rigidity of complete intersections, Math. Z. 285 (2017), no. 1-2, 479-492; https://doi.org/10.1007/s00209-016-1717-7.

Hamid Ahmadinezhad h.ahmadinezhad@lboro.ac.uk

Department of Mathematical Sciences, Loughborough University, Loughborough, LE11 3TU, UK

Takuzo Okada okada@cc.saga-u.ac.jp

Department of Mathematics, Faculty of Science and Engineering, Saga University, Saga 845-8502 Japan 